Universidad Politécnica de Valencia

Departamento de Matemática Aplicada

\title{
Espacios métricos fuzzy definidos por $t$-normas
}

\author{
Memoria presentada por \\ Almanzor Sapena Piera \\ para optar al Grado de Doctor en Matemáticas \\ por la Universidad Politécnica de Valencia \\ Dirigida por los Doctores \\ D. Valentín Gregori Gregori \\ D. Salvador Romaguera Bonilla
}

Gandia, enero 2002 


\section{Índice general}

Introducción general 4

1. Propiedades y ejemplos de espacios métricos fuzzy en el sentido de George y Veeramani

1.1. Introducción . . . . . . . . . . . . . . . . 16

1.2. Antecedentes históricos . . . . . . . . . . . . . . . 20

1.3. El espacio métrico fuzzy de George y Veeramani . . . . . . . 29

1.4. Propiedades de los espacios métricos fuzzy $\ldots \ldots$. . . . . 34

1.5. Ejemplos de espacios métricos fuzzy . . . . . . . . . . . . 47

2. Métricas fuzzy no arquimedianas y estándar. Completación de espacios métricos fuzzy

2.1. Introducción . . . . . . . . . . . . . . . . 60 
2.2. Una propiedad relativa a la métrica fuzzy estándar . . . . . . 62

2.3. Métricas fuzzy no arquimedianas . . . . . . . . . . 65

2.4. Completación de los espacios métricos fuzzy estándar . . 67

3. Continuidad uniforme en espacios métricos fuzzy

3.1. Introducción . . . . . . . . . . . . . . . . . . 74

3.2. Continuidad uniforme en espacios métricos fuzzy . . . . . . . 76

3.3. Continuidad $t$-uniforme en espacios métricos fuzzy . . . . . . . 86

4. Teoremas de punto fijo en espacios métricos fuzzy completos 98

4.1. Introducción . . . . . . . . . . . . . . . . . . . . . . 98

4.2. Contracción en espacios métricos fuzzy . . . . . . . . . . . 100

4.3. Teoremas de punto fijo en espacios métricos fuzzy completos . 104

4.4. Teoremas de punto fijo en espacios métricos fuzzy de Kramosil

y Michalek completos en el sentido de Grabiec . . . . . . . . . 120 


\section{Introducción general}

El presente trabajo está dedicado al estudio de los espacios métricos fuzzy, en el sentido de George y Veeramani. Las líneas siguientes sitúan el trabajo dentro del contexto de la teoría fuzzy.

En 1965, Lofti A. Zadeh [54] introdujo el concepto de conjunto fuzzy, que transformó la mayoría de las ramas de la Ciencia y la Ingeniería (incluida la Matemática). Un conjunto fuzzy se define matemáticamente mediante la asignación a cada elemento de un conjunto un valor posible en el intervalo $[0,1]$ que representa su grado de pertenencia al conjunto fuzzy. Formalmente, dado un conjunto no vacío $X$, se denomina conjunto fuzzy de $X$ a toda aplicación $A: X \rightarrow[0,1]$. Esta noción elimina, de alguna forma, la frontera entre los elementos pertenecientes y los no pertenecientes, introduciendo "borrosidad" en dicha frontera. La aparición de este concepto está motivada por la gran cantidad de situaciones reales en las que los objetos no tienen un criterio totalmente definido de pertenencia. La unión e intersección de una familia de conjuntos fuzzy de $X$, se define como el supremo e ínfimo, respectivamente, de la familia de funciones.

Uno de los primeros campos fuzzy que aparece en la Matemática fue el de la Topología Fuzzy, iniciada por C. L. Chang en 1968 [7]. Para Chang, 
una topología $\tau$ en $X$ es una familia de conjuntos fuzzy de $X$ cerrada para uniones cualesquiera, y para intersecciones finitas, que contiene las funciones constantes 0 y 1 . Aunque éste es el concepto mayormente utilizado por los autores que posteriormente han desarrollado la teoría, no es el único. Así, en 1976, R. Lowen [36], [37], exige además que $\tau$ contenga todas las funciones constantes; a esta topología en [49] se la denomina laminada. En consecuencia, la topología laminada no constituye una generalización de la topología, en sentido clásico, a diferencia de la de Chang. Por otra parte, Goguen [18] y Hutton [31], generalizan la noción de Chang reemplazando el rango $I=[0,1]$ de los conjuntos fuzzy, por un retículo complementado $L$, dando lugar al concepto de $L$-topología.

En los anteriores casos los miembros de $\tau$, que se denominan abiertos, carecen de graduación. Esta situación cambia en la estructura topológica fuzzy que define A. Šostak [48], para el cual una topología fuzzy es una aplicación $\sigma: I^{X} \rightarrow I$, que verifica ciertos axiomas. A la misma noción, tras ligeras modificaciones, [8], [29], llegaron Hazra et al., denominando graduación de abiertos a la aplicación $\sigma$.

Recientemente K. Atanassov [2], [3], [4] ha introducido el concepto de conjunto intuicionista, que generaliza el de conjuto fuzzy y que ha sido usado por D. Çoker [9] para definir el concepto de espacio topológico intuicionista, como una generalización del debido a Chang.

Entre los problemas más interesantes que se han suscitado en la Topología Fuzzy, destaca el de obtener una noción adecuada de espacio métrico fuzzy. Recordemos que el estudio de los espacios métricos se basa en la noción de distancia entre puntos, pero en muchas ocaciones reales, esta distancia no puede determinarse con exactitud. Este problema, que pertence al ámbito fuzzy, había sido anteriormente abordado por la teoría de la probabilidad. 
En efecto, en 1942, K. Menger [38] introdujo los llamados espacios métricos probabilísticos, donde si $d(x, y)$ es la distancia entre los puntos $x$ e $y$, entonces la función de distribución $F_{x y}(t)$ representa la probabilidad de que la distancia entre $x$ e $y$ sea menor o igual que $t$. Posteriormente, Schweizer y Sklar [45], [46], continuaron el desarrollo de estos espacios y, recientemente han sido muchos los trabajos aportados en este sentido [6], [40], [41], [42], [45], [51], etc.

En los párrafos anteriores hemos visto que la noción de Topología Fuzzy ha sido tratada desde diversos puntos de vista, lo cual es casi una constante en cuantos conceptos fuzzy se definen. En lo que respecta a las métricas fuzzy, también han sido numerosos autores los que han abordado este concepto de distintas maneras, que nosotros, simplificando, dividimos en dos grandes grupos. El primero estaría formado por aquellos trabajos en los cuales una (pseudo-)métrica en $X$ es tratada como una función $d: \Omega \times \Omega \longrightarrow \mathbb{R}$ con $\Omega \subset I^{X}(I=[0,1])$ satisfaciendo algunos axiomas "análogos" al caso clásico. Entre éstos podemos destacar los trabajos de Deng Zi-ke [10], Erceg [15], $\mathrm{Hu}$ [30], y Artico y Moresco [1]. Los principales problemas de interés en esta línea son: de qué manera una métrica fuzzy induce una (quasi-) uniformidad fuzzy (en el sentido de [32]) y una topología fuzzy [15], [10], [30]; criterios de (pseudo-) metrización [14], [15], [30]; propiedades de separación en espacios métricos [15], [1], [30], [11] y propiedades de completación y acotación [1], [11]. En el segundo grupo incluiríamos aquellos artículos en los cuales la distancia entre objetos es fuzzy. Los más relevantes en esta dirección son los debidos a Kaleva y Seikkala [33], Eklund y Gähler [13], y Kramosil y Michalek [35]. El concepto de métrica fuzzy objeto de nuestro estudio es el debido a George y Veeramani [19], [20], que constituye una modificación del concepto de métrica fuzzy introducida por Kramosil y Michalek. 
George y Veeramani probaron que toda métrica fuzzy (en su sentido) genera una topología, y que de todo espacio métrico (clásico) podía deducirse un espacio métrico fuzzy, denominado estándar. Pensamos que la definición de métrica fuzzy que dieron estos autores es adecuada y merece especial atención porque, como demostraron Gregori y Romaguera [24], la clase de los espacios topológicos fuzzy que son metrizables coincide con la de los metrizables fuzzy (en sentido obvio) de George y Veeramani.

En esta memoria hemos proseguido con el estudio de los espacios métricos fuzzy de George y Veeramani y hemos aportado nuevos ejemplos y propiedades de estos espacios. Además hemos tratado temas como la completación, la continuidad uniforme, y teoremas de punto fijo. Aunque cada uno de los cuatro capítulos de que consta el trabajo va precedido de una pequeña introducción, veamos a continuación los conceptos y resultados más relevantes que hemos obtenido en cada uno de ellos.

El capítulo 1 comienza con un pequeño resumen histórico de los antecedentes que llevaron a la teoría de los espacios métricos fuzzy, empezando con la definición 1.2.1 de espacio métrico probabilístico debida a Menger, y la debida a Schweizer y Sklar (definición 1.2.5) en la que por primera vez aparece el concepto de $t$-norma continua (definición 1.2.4). Después presentamos la definición 1.2.7 de espacio métrico (fuzzy) de Kaleva y Seikkala que está basada en la definición de número fuzzy, y finalmente la debida a Kramosil y Michalek (definición 1.2.10). Sobre esta última se fundamenta la siguiente definición de George y Veeramani, con la que empieza la tercera sección del capítulo, y que contiene los preliminares para el resto del trabajo, que pueden verse en [19], [20].

La terna $(X, M, *)$ es un espacio métrico fuzzy si $X$ es un conjunto arbitrario, $*$ es una operación binaria continua en $[0,1]$ que es conmutati- 
va, asociativa, con elemento neutro y compatible con el orden del intervalo $[0,1]$ (esta operación se llama $t$-norma continua) y $M$ es un conjunto fuzzy en $X \times X \times] 0,+\infty[$ que satisface los siguientes axiomas, para cualesquiera $x, y, z \in X, t, s>0$ :

(GV1) $M(x, y, t)>0$

(GV2) $M(x, y, t)=1$ si y sólo si $x=y$

$(\mathrm{GV} 3) M(x, y, t)=M(y, x, t)$

(GV4) $M(x, y, t) * M(y, z, s) \leq M(x, z, t+s)$

(GV5) $M(x, y,):.] 0,+\infty[\longrightarrow] 0,1]$ es continua

En lo que sigue, por espacio métrico fuzzy entenderemos el debido a George y Veeramani, y como es usual, nombraremos, por ejemplo, espacio métrico fuzzy $X$ sin mención a la métrica fuzzy, si no es necesario.

La métrica fuzzy $M$ genera una topología en $X$, que denotaremos $\tau_{M}$, que tiene por base la familia de bolas abiertas $\left\{B_{M}(x, r, t): x \in X, 0<r<\right.$ $1, t>0\}$ donde $B_{M}(x, r, t)=\{y \in X: M(x, y, t)>1-r\}$. Una sucesión $\left\{x_{n}\right\}_{n=1}^{\infty}$ se dice de Cauchy, si para cada $\left.\varepsilon \in\right] 0,1\left[\right.$ y $t>0$, existe $n_{0} \in \mathbb{N}$ de manera que $M\left(x_{n}, x_{m}, t\right)>1-\varepsilon$ siempre que $m, n \geq n_{0}$, y $X$ es completo si las sucesiones de Cauchy convergen. Se dice que $X$ es $F$-acotado si existe $r \in] 0,1[$ de manera que $M(x, y, t)>1-r$ para cualesquiera $x, y \in X, t>0$.

Si $(X, d)$ es un espacio métrico, entonces la función $M_{d}(x, y, t)=\frac{t}{t+d(x, y)}$ es una métrica fuzzy en $X$, con la $t$-norma producto, y la topología $\tau_{M_{d}}$ coincide con la inducida por $d$. Las anteriores definiciones fuzzy son acordes (expresión que utilizaremos en situaciones análogas) con la teoría clásica de los espacios métricos, en el sentido de que $(X, d)$ es completo (acotado) si y sólo si $\left(X, M_{d}, \cdot\right)$ es completo ( $F$-acotado). 
En la sección 4 del capítulo 1 obtenemos, entre otros resultados, nuevas métricas a partir de otras dadas, y demostramos que todo espacio métrico fuzzy precompacto (definición 1.4.12, que es acorde con la teoría de los espacios métricos, según la proposición 1.4.18) $(X, M, *)$ en el cual $a * b \neq 0$ si $a, b \neq 0$, es $F$-acotado. Queda como problema abierto probar si la precompacidad fuzzy implica $F$-acotación. Tratar de establecer diferencias esenciales entre la teoría de los espacios métricos fuzzy y la teoría clásica de los espacios métricos es tarea difícil, dado que todos los ejemplos de métrica fuzzy, salvo uno, dados en [19], [20], se deducen, de alguna manera, de métricas ordinarias, y como consecuencia de ello heredan sistemáticamente las correspondientes propiedades métricas. Por esta razón, la última sección del primer capítulo la dedicamos a analizar con detalle los ejemplos de métrica fuzzy existentes (a la vez que generalizamos algunos ejemplos y corregimos algunos errores de [19]) según que la $t$-norma considerada sea $T_{1}(x, y)=\operatorname{mín}\{x, y\}$ o $T_{2}(x, y)=x y$. De mayor interés son los nuevos ejemplos que aportamos de métricas fuzzy, que resultan serlo para la $t$-norma $T_{3}(x, y)=\operatorname{máx}\{0, x+y-1\}$, pero no lo son para $T_{1}$ ni $T_{2}$. Especialmente relevante es la métrica fuzzy del ejemplo 1.5.12 con la $t$-norma continua $T_{3}$ definida en el intervalo $] 2,+\infty[$, por la función

$$
M(x, y, t)=\left\{\begin{array}{cr}
1-\left(\frac{1}{x \wedge y}-\frac{1}{x \vee y}\right) & \text { si } \quad x, y \in A \text { ó } x, y \in B \\
\frac{1}{x}+\frac{1}{y} & \text { en los demás casos }
\end{array}\right.
$$

donde $\{A, B\}$ es una partición no trivial de $] 2,+\infty[$, pues se trata de una modificación (y en la práctica una generalización) de la métrica fuzzy dada por Gregori y Romaguera en [25], que como se prueba en el capítulo 2, no admite "completación", finalidad por la que fue ideada por los autores. 
Parte de los resultados de este capítulo han sido publicados en [43].

En el segundo capítulo definimos el concepto de métrica fuzzy no arquimediana $M$ como aquélla que verifica $M(x, z, t) \geq \operatorname{mín}\{M(x, y, t), M(y, z, t)\}$, la cual resulta acorde con la teoría clásica. Este nuevo concepto fuzzy permite obtener el siguiente teorema 2.3.4 de caracterización: Un espacio topológico metrizable $(X, \tau)$ es fuertemente 0 -dimensional si y sólo si $\tau$ está generada por una métrica fuzzy no arquimediana.

La completación métrica fuzzy se aborda en la última sección. Hasta ahora, y a excepción de la precompacidad, hemos visto cómo los conceptos acerca de métricas fuzzy llevaban a propiedades que resultaban ser "análogas" a las de las métricas ordinarias. En el caso de la completación, la situación es muy distinta. En efecto, como ya hemos mencionado, Gregori y Romaguera [24] han construido una métrica fuzzy (ejemplo 1.5.12) que no admite completación métrica fuzzy, donde por completación métrica fuzzy del espacio $(X, M, *)$ se entiende una cuaterna $(Y, N, \star, f)$ donde $(Y, N, \star)$ es un espacio métrico fuzzy completo y $f$ una isometría fuzzy (i.e., una aplicación de $X$ en $Y$ que "conserva" las distancias fuzzy) de $X$ en un conjunto denso de $Y$. Nosotros hemos dado una prueba corta de que el espacio del ejemplo 1.5.12 no admite completación, por medio del lema 2.4.5, que establece que si $\left\{a_{n}\right\}_{n=1}^{\infty}$ y $\left\{b_{n}\right\}_{n=1}^{\infty}$ son sucesiones de Cauchy en un espacio $(X, M, *)$ que admite completación, entonces para cualquier $t>0$, la sucesión $\left\{M\left(a_{n}, b_{n}, t\right)\right\}_{n=1}^{\infty}$ no puede converger a cero.

El capítulo acaba mostrando que si $(X, d)$ es un espacio métrico, entonces el correspondiente espacio métrico fuzzy estándar $\left(X, M_{d}, T_{2}\right)$ admite como completación el espacio métrico fuzzy estándar $\left(\tilde{X}, M_{\tilde{d}}, T_{2}\right)$, donde $\tilde{d}$ designa 
la completación métrica de $d$. Esta completación es única (salvo isometría fuzzy) pues en el teorema 2.4.7 demostramos que si $M$ es una métrica fuzzy, continua respecto de $x, y$, que admite completación, entonces ésta es única, salvo isometría fuzzy (obviamente $M_{d}$ satisface las hipótesis del teorema).

El capítulo 3 se dedica al estudio de la continuidad uniforme. Si $(X, M, *)$ e $(Y, N, \star)$ son espacios métricos fuzzy, la aplicación $f: X \rightarrow Y$ se dice que es uniformemente continua si para cada $\varepsilon \in] 0,1[\mathrm{y} t>0$, existen $r \in] 0,1[\mathrm{y}$ $s>0$, de manera que $M(x, y, s)>1-r$ implica $N(f(x), f(y), t)>1-\varepsilon$ para cualesquiera $x, y \in X$. Además, diremos que la métrica fuzzy $M$ es equinormal si para cada par de cerrados disjuntos, no vacíos, $A$ y $B$ de $X$, existe $t>0$ tal que $\sup \{M(a, b, t): a \in A, b \in B\}<1$, y que $M$ tiene la propiedad de Lebesgue si para cada cubrimiento abierto $\mathcal{G}$ de $X$ existen $r \in] 0,1\left[\right.$ y $s>0$ de manera que $\left\{B_{M}(x, r, s): x \in X\right\}$ refina a $\mathcal{G}$. Una vez más los conceptos dados son acordes con la teoría de los espacios métricos, según 3.2.6 y 3.2.8. El resultado fundamental de este capítulo es el teorema 3.2 .9 en el que se caracterizan los espacios métricos fuzzy $(X, M, *)$ donde las funciones reales continuas en $\left(X, \tau_{M}\right)$ son uniformemente continuas, por el hecho de que $M$ es equinormal (tiene la propiedad de Lebesgue) o que $\mathcal{U}_{M}$ es equinormal (tiene la propiedad de Lebesgue), siendo $\mathcal{U}_{M}$ la uniformidad inducida por $M$ que se construye en la prueba del teorema 1.3.15.

En la tercera sección de este capítulo introducimos y estudiamos el siguiente concepto más fuerte que el de continuidad uniforme: la aplicación $f:(X, M, *) \rightarrow(Y, N, \star)$ es $t$-uniformemente continua si para cada $\varepsilon \in] 0,1[$ existe $r \in] 0,1[$ de manera que $M(x, y, t)>1-r$ implica $N(f(x), f(y), t)>$ $1-\varepsilon$ para cualesquiera $x, y \in X, t>0$. Este concepto no tiene "homólogo" en los espacios métricos clásicos pero está estrechamente relacionado con 
el concepto de contractividad fuzzy del próximo capítulo. En la proposición 3.3.4 probamos que si $f:(X, M, *) \rightarrow(Y, N, \star)$ es continua y $X$ es compacto, entonces $f$ es $t$-uniformemente continua.

De manera similar a la sección anterior, aquellos espacios métricos fuzzy $(X, M, *)$ donde toda función real continua es $t$-uniformemente continua de $(X, M, *)$ en $\left(\mathbb{R}, M_{|\cdot|}, T_{2}\right)$ vienen caracterizados porque la métrica $M$ es $t$ equinormal (i.e., para cada par de cerrados disjuntos, no vacíos, $A$ y $B$ y para todo $t>0, \sup \{M(a, b, t): a \in A, b \in B\}<1$, definición 3.3.5). Para ilustrar los resultados de este capítulo, en la proposición 3.3.7 se construye un espacio métrico fuzzy $X$, no compacto, que resulta ser equinormal, y se da una función real en $X$ que es uniformemente continua pero no es $t$ uniformemente continua. (En la proposición 3.3.11 se ofrece otro ejemplo). Finalmente, en 3.3.12 se prueba que si $(X, M, *)$ es un espacio métrico fuzzy en el que toda función real continua es uniformemente continua de $(X, M, *)$ en $\left(\mathbb{R}, M_{|\cdot|}, T_{2}\right)$, entonces existe una métrica fuzzy $(N, \star)$ en $X$ compatible con $\tau_{M}$, para la que toda función real continua en $X$ es $t$-uniformemente continua de $(X, M, *)$ en $\left(\mathbb{R}, M_{|\cdot|}, T_{2}\right)$.

Parte de los resultados de este capítulo han sido publicados en [26].

El último capítulo lo dedicamos a la obtención de teoremas de punto fijo, para funciones contractivas fuzzy.

Una aplicación $f$ del espacio métrico fuzzy $(X, M, *)$ en si mismo, diremos que es contractiva fuzzy si existe $k \in] 0,1[$ tal que

$$
\frac{1}{M(f(x), f(y), t)}-1<k\left(\frac{1}{M(x, y, t)}-1\right)
$$


Éste es un concepto más fuerte que el de aplicación $t$-uniformemente continua como se prueba en 4.2.2, y además es acorde con la teoría clásica de los espacios métricos (4.2.3). Una sucesión $\left\{x_{n}\right\}_{n=1}^{\infty}$ en $(X, M, *)$ decimos que es contractiva fuzzy si existe $k \in] 0,1[$ de manera que

$$
\frac{1}{M\left(x_{n+1}, x_{n+2}, t\right)}-1<k\left(\frac{1}{M\left(x_{n}, x_{n+1}, t\right)}-1\right)
$$

para todo $n \in \mathbb{N}, t>0$, y de nuevo resulta ser un concepto acorde con la teoría clásica. En el teorema 4.3.1 establecemos que toda aplicación contractiva fuzzy en un espacio métrico fuzzy completo, donde las sucesiones contractivas fuzzy son de Cauchy, posee un único punto fijo. Como corolario se obtiene lo que podríamos denominar versión fuzzy del teorema de contractividad de Banach: Si $f$ es una aplicación contractiva fuzzy en un espacio métrico fuzzy estándar completo, entonces $f$ posee un único punto fijo. El teorema 4.3.1 se generaliza en 4.3.4 a una familia numerable de aplicaciones contractivas fuzzy.

Decimos que una sucesión de reales positivos $\left\{t_{n}\right\}_{n=1}^{\infty}$ es $s$-creciente si existe $n_{0} \in \mathbb{N}$ de manera que $t_{n}+1 \leq t_{n+1}$, para $n \geq n_{0}$. El otro resultado fundamental de este capítulo es otra versión fuzzy del teorema de contractividad de Banach, que en su forma más sencilla dice: Sea $(X, M, *)$ un espacio métrico fuzzy completo, en el que para cada $\varepsilon>0, x, y \in X$, y cada sucesión $\left\{t_{n}\right\}_{n=1}^{\infty} s$-creciente, se tiene que el producto infinito

$$
\prod_{n=1}^{\infty} M\left(x, y, t_{n}\right)
$$

converge. Sea $T$ una aplicación de $X$ en si mismo. Si existe $k \in] 0,1[$ de manera que se satisface la condición de contractividad $M(T(x), T(y), k t) \geq M(x, y, t)$ (debida a Sehgal y Bahrucha-Reid [47]), entonces $T$ posee un único punto fijo. En la misma sección 3, se incluyen algunos ejemplos que ilustran la necesidad de algunas de las hipótesis de los anteriores teoremas. 
Excepcionalmente, en la última sección, $(X, M, *)$ es un espacio métrico fuzzy en el sentido de Kramosil y Michalek. Una sucesión $\left\{x_{n}\right\}_{n=1}^{\infty}$ en $X$ se dice $G$-Cauchy (en sentido Grabiec) si $\lim _{n \rightarrow \infty} M\left(x_{n+p}, x_{n}, t\right)=1$ para cualesquiera $t>0, p \in \mathbb{N}$. El resultado fundamental de esta sección establece que si $T$ es una aplicación contractiva fuzzy en el espacio métrico fuzzy $G$ completo $X$, entonces $T$ posee un único punto fijo. La misma tesis se tiene si reemplazamos la hipótesis de que el espacio es $G$-completo por compacto (aquél en el que toda sucesión posee un subsucesión convergente), lo que constituye una versión del teorema de contractividad de Edelstein.

Parte de los resultados de este capítulo han sido aceptados para su publicación en [27]. 


\section{Capítulo 1}

\section{Propiedades y ejemplos de espacios métricos fuzzy en el}

sentido de George y Veeramani

\subsection{Introducción}

El concepto de espacio métrico fuzzy que nosotros adoptaremos es el debido a George y Veeramani, que es una modificación del debido a Kramosil y Michalek. A continuación veremos algunos resultados en la teoría de estos autores, porque son precedentes y, a la vez, han sido guía para los obtenidos posteriormente por George y Veeramani, y por nosotros mismos, sobre todo en el capítulo último dedicado al estudio de puntos fijos en aplicaciones contractivas. También daremos a conocer el concepto de espacio métrico fuzzy debido a Kaleva y Seikkala por su relación con los espacios de Menger. Pero antes de desarrollar ambas teorías haremos un pequeño sumario cronológico 
con la aparición de los diversos conceptos y resultados relevantes. Usualmente denominaremos funciones a las aplicaciones cuyo espacio de llegada es el conjunto de los números reales.

En el año 1942, Menger [38] introdujo un espacio al cual se le asocia una función de distribución que actúa sobre cualquier par de elementos del espacio. Si $d(x, y)$ es la distancia entre los puntos $x$ e $y$, entonces la función de distribución $F_{x y}(t)$, para cualquier número real $t$, se interpreta como la probabilidad de que la distancia entre $x$ e $y$ sea menor o igual que $t$.

El concepto fuzzy se introdujo también en la teoría de espacios métricos probabilísticos. La razón principal de ello ha sido que la incertidumbre acerca de la distancia entre dos puntos está más cerca del concepto fuzzy que de la aleatoriedad. En 1984, Kaleva y Seikkala [33] definieron el concepto de métrica fuzzy generalizando la noción de métrica y probaron que todo espacio probabilístico de Menger puede considerarse un espacio métrico fuzzy. Utilizando la definición de métrica fuzzy de Kaleva y Seikkala, Hadzic [28] probó teoremas de punto fijo para aplicaciones multiformes en algunas clases de espacios métricos fuzzy.

En otra línea de investigación, Kramosil y Michalek [35] generalizaron el concepto de espacio métrico probabilístico a la nueva teoría fuzzy. Han sido demostrados algunos teoremas de punto fijo basados en la definición de espacio métrico fuzzy aportado por Kramosil y Michalek. En 1989, Grabiec [23] introdujo una noción de sucesión de Cauchy en estos espacios y probó una versión del teorema de Banach, adaptado a la teoría fuzzy, y el teorema de punto fijo de Edelstein. Posteriormente, R. Vasuki generalizó un teorema de Grabiec a una sucesión de aplicaciones [52]. En 1992, Fang [16] demostró varios teoremas de punto fijo en dichos espacios métricos. En 1994, Mishra, Sharma y Singh [39] obtuvieron teoremas de punto fijo común para apli- 
caciones contractivas y aplicaciones asintóticamente conmutativas en dichos espacios métricos fuzzy. Subrahmanyam [50] generalizó el teorema de punto fijo de Jungck a los espacios métricos fuzzy de Kramosil y Michalek.

Posteriormente, George y Veeramani [19, 20] modificaron la definición de espacio métrico fuzzy introducida por Kramosil y Michalek. Ello fue motivado por el hecho de que la topología deducida de la métrica de Kramosil y Michalek no resulta ser una topología Hausdorff. Consiguientemente, George y Veeramani dieron una nueva noción de métrica fuzzy (o de espacio métrico fuzzy) que permitía deducir una topología Hausdorff en el espacio que, además, cumple el Primer Axioma de Numerabilidad (definición 1.3.1). A partir de la tercera sección nosotros adoptaremos esta definición. Por otra parte, dichos autores modificaron la noción de sucesión de Cauchy introducida por Grabiec puesto que probaron que el espacio métrico fuzzy estándar deducido del espacio métrico usual no resultaba ser completo en el sentido de Grabiec. Con esta nueva definición los autores probaron que dicho espacio es completo. Finalmente, Gregori y Romaguera [24] y los propios autores George y Veeramani [21], [22] han proseguido el estudio de dichos espacios. Gregori y Romaguera [24], demostraron que la clase de los espacios topológicos que son fuzzy metrizables (en el sentido de George y Veeramani) coincide con la clase de los espacios topológicos metrizables. Este resultado es interesante puesto que ello permite trasladar muchos de los conceptos y resultados, digamos, clásicos, de la teoría de espacios métricos a la teoría de los espacios métricos fuzzy. No obstante, la escasez de ejemplos conocidos de métricas fuzzy ha venido impidiendo encontrar diferencias significativas entre la teoría de los espacios métricos y la teoría de los espacios métricos fuzzy, en el sentido de George y Veeramani, dado que, prácticamente todos los ejemplos conocidos de métricas fuzzy (en realidad, todos menos uno), venían deducidos, de alguna manera, de métricas usuales, y ello hacía que sus propiedades fueran, 
en gran parte, heredadas.

El objetivo de este capítulo, que consta de 4 secciones, es, pues, generalizar algunos de los ejemplos aportados por George y Veeramani y, además, introducir otros ejemplos de métricas fuzzy que no son deducidos de métrica alguna, lo que nos va a permitir, en posteriores investigaciones, profundizar en el estudio de estos espacios métricos. También estudiaremos algunas propiedades de las métricas fuzzy, en particular algunas referentes a acotación. La estructura del capítulo es la siguiente. En la sección 3 introducimos la definición de espacio métrico fuzzy en el sentido de George y Veeramani y damos las primeras propiedades asociadas a este concepto. Entre ellas, la proposición 1.3.5 aporta la definición de métrica fuzzy estándar asociada a una métrica. A continuación, la nota 1.3.8, introduce la topología generada por una métrica fuzzy. Además, la definición 1.3.9 da el concepto de sucesión de Cauchy en un espacio métrico fuzzy y, por último, la proposición 1.3.11 relaciona un espacio métrico con su espacio métrico fuzzy estándar asociado. En la cuarta sección, tomando tres t-normas continuas (que llamare$\operatorname{mos} T_{1}(x, y)=\operatorname{mín}\{x, y\}, T_{2}(x, y)=x y$ y $\left.T_{3}(x, y)=\operatorname{máx}\{x+y-1,0\}\right)$, se dan varias proposiciones relativas a la construcción de nuevas métricas fuzzy, a partir de otras dadas. Concretamente, la proposición 1.4.4 demuestra que si $M(x, y, t)$ es una métrica fuzzy en un conjunto $X$, entonces la función $N(x, y, t)=\frac{k+M(x, y, t)}{1+k}$ es una métrica fuzzy (siendo $\left.k \in\right] 0,1[$ ). Por su parte, la proposición 1.4.5 prueba que, salvo algún caso, el producto de métricas fuzzy (entendiéndose como producto la $t$-norma continua común a ambas) es una métrica fuzzy. Además, la proposición 1.4.9 aclara que el mínimo de dos métricas fuzzy también lo es. Para terminar esta sección, en la definición 1.4.12 se introduce el concepto de métrica fuzzy precompacta y en la proposición 1.4.19 se demuestra que si $(M, *)$ es una métrica fuzzy pre- 
compacta (con una pequeña condición adicional) entonces el espacio métrico fuzzy $(X, M, *)$ es $F$-acotado. La sección 4 está dedicada a la enumeración de varios ejemplos de métricas fuzzy no estándar. En algunos casos, como en los ejemplos 1.5 .3 y 1.5.5, esta definición no depende de la $t$-norma continua asociada. Ahora bien, se dan ejemplos en los que la $t$-norma continua asociada a la métrica fuzzy juega un papel relevante puesto que las condiciones se cumplen o no dependiendo de cuál sea dicha t-norma continua. El ejemplo 1.5.7 muestra un espacio métrico fuzzy cuya $t$-norma asociada es $T_{2}$ pero que deja de serlo si la $t$-norma asociada es $T_{1}$. Por su parte, los ejemplos 1.5.10, 1.5.11 y 1.5.12, muestran nuevos espacios métricos fuzzy con $t$-norma asociada $T_{3}$ que no lo son con la $t$-norma asociada $T_{2}$. Mención especial merece el ejemplo 1.5.12 por su complejidad. En él se demuestra que $\left(X, M, T_{2}\right)$ es un espacio métrico fuzzy, siendo $\{A, B\}$ una partición no trivial de $X=] 0,+\infty[$ y $M$ definida por

$$
M(x, y, t)=\left\{\begin{array}{cc}
1-\left(\frac{1}{x \wedge y}-\frac{1}{x \vee y}\right) & \text { si } x, y \in A \text { ó } x, y \in B \\
\frac{1}{x}+\frac{1}{y} & \text { en los demás casos }
\end{array}\right.
$$

Este ejemplo ha sido utilizado para probar, en el siguiente capítulo, que no todo espacio métrico fuzzy admite completación.

\subsection{Antecedentes históricos}

\section{Espacios métricos probabilísticos}

Antes de que la teoría fuzzy fuera introducida en el campo de los espacios métricos, el estudio de métricas estaba asociado a nociones de probabilidad. 
En este sentido, en 1942, Menger [38] definió el concepto de espacio métrico probabilístico de la siguiente forma.

Definición 1.2.1. Sea $X$ un conjunto arbitrario no vacío. Sea $F_{p q}$ una familia de funciones de distribución que satisfacen las siguientes condiciones:

(M1) $F_{p q}(0)=0$

(M2) Si $p=q$, entonces $F_{p q}(x)=1$ para todo $x>0$

(M3) Si $p \neq q$, entonces $F_{p q}(x)<1$ para algún $x>0$

(M4) $F_{p q}=F_{q p}$

(M5) $F_{p r}(x+y) \geq T\left(F_{p q}(x), F_{q r}(y)\right)$ para cualesquiera $p, q, r \in X y$ cualesquiera $x, y \in \mathbb{R}$, siendo $T:[0,1] \times[0,1] \rightarrow[0,1]$ una función que satisface:

(i) $T(a, b)=T(b, a)$

(ii) $T(a, b) \leq T(c, d)$ siempre que $a \leq c$ y $b \leq d$

(iii) $T(a, 1)>0$ siempre que $a>0, y T(1,1)=1$

Recordemos que una función de distribución $F: \mathbb{R} \rightarrow[0,1]$ es una aplicación continua por la izquierda y no decreciente tal que $\inf _{x \in X}\{F(x)\}=0 \mathrm{y}$ $\sup _{x \in X}\{F(x)\}=1$. La métrica estadística $F_{p q}$ puede ser interpretada como la probabilidad de que la distancia entre dos puntos $p$ y $q$ sea menor que $x$.

A partir de esta definición se fueron introduciendo algunas modificaciones que generalizaban este tipo de espacios métricos.

Nota 1.2.2. Wald ([46], p. 4) sugirió reemplazar la desigualdad (M5) por

$$
F_{p r}(x) \geq\left(F_{p q} * F_{q r}\right)(x)
$$


donde * denota el producto de convolución, es decir:

$$
\left(F_{p q} * F_{q r}\right)(x)=\int_{-\infty}^{+\infty} F_{p q}(x-y) d F_{q r}(y)=\int_{0}^{x} F_{p q}(x-y) d F_{q r}(y)
$$

El espacio resultante se denomina espacio de Wald.

Nota 1.2.3. Schweizer y Sklar [46] reemplazaron la condición (M5) por la siguiente:

$$
\text { Si } F_{p q}(x)=1 \text { y } F_{q r}(y)=1 \text {, entonces } F_{p r}(x+y)=1
$$

El espacio resultante se llama espacio métrico probabilístico débil, que generaliza los espacios probabilísticos de Menger y los espacios de Wald.

Schweizer y Sklar, introdujeron el concepto de $t$-norma continua definido como sigue y que tendrá una importante relevancia en el desarrollo del presente trabajo.

Definición 1.2.4. Una operación binaria $*:[0,1] \times[0,1] \rightarrow[0,1]$ se dice que es una t-norma continua si satisface la siguientes condiciones:

(i) * es continua, asociativa y conmutativa

(ii) $a * 1=$ a para todo $a \in[0,1]$

(iii) $a * b \leq c * d$ siempre que $a \leq c$ y $b \leq d, a, b, c, d \in[0,1]$

A partir de esta definición, Schweizer y Sklar definieron espacio métrico probabilístico de la siguiente forma.

Definición 1.2.5. Un espacio métrico probabilístico es un par $(X, F)$ donde $X$ es un conjunto arbitrario y $F$ es una aplicación de $X \times X$ en el conjunto de todas las funciones de distribución que satistace las siguientes propiedades: 
(1) $F_{x y}(t)=1$ para todo $t>0$ si $y$ sólo si $x=y$

(2) $F_{x y}(0)=0$

(3) $F_{x y}=F_{y x}$

(4) Si $F_{x y}(t)=1$ y $F_{y z}(s)=1$, entonces $F_{x z}(t+s)=1$

Un espacio de Menger $(X, F, *)$ es un espacio métrico probabilístico junto con una t-norma continua que satisface la condición

$$
F_{x z}(t+s) \geq F_{x y}(t) * F_{y z}(s)
$$

\section{Espacios métricos fuzzy de Kaleva y Seikkala}

Dado que la incertidumbre acerca de la distancia entre dos objetos está más relacionada con la noción fuzzy que con la aleatoriedad, Kaleva y Seikkala [33] extendieron el concepto de espacio métrico a la nueva teoría fuzzy asociando la distancia entre dos puntos a un número fuzzy.

Definición 1.2.6. Un número fuzzy es una aplicación $x: \mathbb{R} \rightarrow[0,1]$ que asocia a cada número real $t$ su grado de pertenencia $x(t)$.

Un número fuzzy $x$ se dice que es convexo si $x(t) \geq \min \{x(s), x(r)\}$ donde $s \leq t \leq r$.

Para $0<\alpha \leq 1$ y un número fuzzy $x$, sus $\alpha$-conjuntos de nivel $[x]_{\alpha}$ están definidos por

$$
[x]_{\alpha}=\{u: x(u) \geq \alpha\}
$$

Con ello, resulta que $x$ es convexo si y sólo si $[x]_{\alpha}$ es un conjunto convexo en $\mathbb{R}$ para todo $\alpha \in] 0,1]$ 
Además, si existe un elemento $u \in \mathbb{R}$ tal que $x(u)=1$, entonces el número fuzzy $x$ se dice que es normal.

Un número fuzzy se dice que es no negativo si $x(u)=0$ para todo $u<0$.

El conjunto de todos los números fuzzy convexos, normales, no negativos y semicontinuos por la derecha se denota por $\mathbb{G}$.

Utilizando todos estos preliminares, Kaleva y Seikkala definen el concepto de espacio métrico fuzzy de la siguiente forma.

Definición 1.2.7. Sea $X$ un conjunto arbitrario no vacío, sea $d: X \times X \rightarrow \mathbb{G}$ una aplicación y sean $L, R:[0,1] \times[0,1] \rightarrow[0,1]$ dos aplicaciones simétricas, no decrecientes en ambos argumentos y que, además, satisfacen $L(0,0)=0$ y $R(1,1)=1$. Denotamos

$$
[d(x, y)]_{\alpha}=\left[\lambda_{\alpha}(x, y), \rho_{\alpha}(x, y)\right] \text { para } x, y \in X, 0<\alpha \leq 1
$$

La cuaterna $(X, d, L, R)$ se denomina KS-espacio métrico fuzzy y d se dice que es una KS-métrica fuzzy si se cumple:

(a) $d(x, y)=0$ si $y$ sólo si $x=y$

(b) $d(x, y)=d(y, x)$ para cualesquiera $x, y \in X$

(c) para cualesquiera $x, y, z \in X$,

(1) $d(x, y)(s+t) \geq L(d(x, z)(s), d(z, y)(t))$ siempre que $s \leq \lambda_{1}(x, z), t \leq \lambda_{1}(z, y)$ y $s+t \leq \lambda_{1}(x, y)$

(2) $d(x, y)(s+t) \leq R(d(x, z)(s), d(z, y)(t))$ siempre que $s \geq \lambda_{1}(x, z), t \geq \lambda_{1}(z, y)$ y $s+t \geq \lambda_{1}(x, y)$

Puesto que los números reales no negativos pertenecen a $\mathbb{G}$, tomando

$$
L(a, b)=0 \text { y } R(a, b)= \begin{cases}0 & a=b=0 \\ 1 & \text { en otro caso }\end{cases}
$$


resulta que el espacio métrico usual puede ser considerado como un KSespacio métrico fuzzy. Además, la definición 1.2.7 generaliza los espacios de Menger, como prueba la siguiente nota.

Nota 1.2.8. Sea $(X, F, *)$ un espacio de Menger. Definimos $d: X \times X \rightarrow \mathbb{G}$ como

$$
d(x, y)(t)= \begin{cases}0 & t<t_{x y}=\sup \left\{t: F_{x y}(t)=0\right\} \\ 1-F_{x y}(t) & t \geq t_{x y}\end{cases}
$$

Si tomamos $R(a, b)=1-((1-a) *(1-b))$ y $L(a, b)=0$, entonces $(X, d, L, R)$ es un KS-espacio métrico fuzzy $y$, por tanto, un espacio de Menger puede ser considerado como un KS-espacio métrico fuzzy.

Por otra parte, el recíproco no es cierto en general, aunque $(X, d, L, R)$ puede ser considerado como un espacio de Menger teniendo en cuenta las siguientes condiciones:

$$
\begin{gathered}
\lim _{u \rightarrow \infty} d(x, y)(u)=0 \text { para todo } x, y \in X \\
R(a, 1)=R(1, a)=1 \text { para todo } a \in[0,1]
\end{gathered}
$$

Si estas condiciones se satisfacen, entonces $(X, F, *)$ es un espacio de Menger, donde $a * b=1-R(1-a, 1-b)$ para cualesquiera $a, b \in[0,1], x, y \in$ $X, s \in \mathbb{R}$,

$$
F_{x y}(s)= \begin{cases}0 & s \leq \lambda_{1}(x, y) \\ 1-d(x, y)(s) & s \geq \lambda_{1}(x, y)\end{cases}
$$

Además, $(X, F, *)$ se denomina espacio de Menger asociado.

Teorema 1.2.9. [33] Sea $(X, d, L, R)$ un KS-espacio métrico fuzzy tal que $\lim _{a \rightarrow 0^{+}} R(a, a)=0$. Entonces la familia de conjuntos

$$
\mathcal{U}(\varepsilon, \alpha)=\left\{(x, y) \in X \times X: \rho_{\alpha}(x, y)<\varepsilon\right\}
$$


es base de una uniformidad que induce una topología Hausdorff en X $y$, además, esta topología es metrizable.

El objetivo de encontrar teoremas de punto fijo en este tipo de espacios métricos fuzzy lleva consigo aportar nuevas nociones de convergencia y completitud en dichos espacios. La convergencia en un KS-espacio métrico fuzzy $(X, d, L, R)$ se define como sigue.

Una sucesión $\left\{x_{n}\right\}_{n=1}^{\infty}$ en $X$ se dice que converge a $x \in X$ si

$$
\lim _{n \rightarrow \infty} x_{n}=x \text { si y sólo si } \lim _{n \rightarrow \infty} d\left(x_{n}, x\right)=\overline{0}
$$

Una sucesión $\left\{x_{n}\right\}_{n=1}^{\infty}$ en $X$ se dice que es de Cauchy si

$$
\lim _{m, n \rightarrow \infty} d\left(x_{m}, x_{n}\right)=\overline{0}
$$

Un KS-espacio métrico fuzzy se dice que es completo si toda sucesión de Cauchy en $X$ es una sucesión convergente. En un KS-espacio métrico fuzzy toda sucesión convergente es una sucesión de Cauchy.

\section{Espacios métricos fuzzy de Kramosil y Michalek}

Kramosil y Michalek [35] definieron el concepto de espacio métrico fuzzy generalizando el de espacio métrico probabilístico a la nueva teoría fuzzy de la siguiente forma:

Definición 1.2.10. [23, 35] Se llama espacio métrico fuzzy de Kramosil y Michalek (KM-espacio métrico fuzzy) a una terna $(X, M, *)$ donde $X$ es un conjunto arbitrario, * es una $t$-norma continua y $M$ es un conjunto fuzzy en $X \times X \times[0,+\infty[$ que satisface las siguientes condiciones, para cualesquiera $x, y, z \in X y t, s>0$ : 
(KM1) $M(x, y, 0)=0$

(KM2) $M(x, y, t)=1$ para todo $t>0$ si $y$ sólo si $x=y$

(KMЗ) $M(x, y, t)=M(y, x, t)$

$(K M 4) M(x, y, t) * M(y, z, s) \leq M(x, z, t+s)$

$(K M 5) M(x, y, \cdot):[0,+\infty[\rightarrow[0,1]$ es continua

Del mismo modo que anteriormente, esta definición aporta nuevas nociones de convergencia y completitud.

Definición 1.2.11. [23] Una sucesión $\left\{x_{n}\right\}_{n=1}^{\infty}$ en un KM-espacio métrico fuzzy se dice que es de Cauchy si

$$
\lim _{n \rightarrow+\infty} M\left(x_{n+p}, x_{n}, t\right)=1, \text { para todo } p>0, t>0
$$

Definición 1.2.12. [23] Una sucesión $\left\{x_{n}\right\}_{n=1}^{\infty}$ en un KM-espacio métrico fuzzy se dice que es convergente $a x \in X$ si

$$
\lim _{n \rightarrow+\infty} M\left(x_{n}, x, t\right)=1, \text { para todo } t>0
$$

Definición 1.2.13. [23] Un KM-espacio métrico fuzzy $(X, M, *)$ se dice que es completo si toda sucesión de Cauchy es convergente y se dice que es compacto si toda sucesión contiene una subsucesión convergente.

Utilizando el concepto de KM-espacio métrico fuzzy, Grabiec [23] continuó el estudio de estos espacios y dio una noción de contractividad en dichos espacios. Con esta definición consiguió demostrar un teorema de contracción de Banach y un teorema de punto fijo de Edelstein, en KM-espacios métricos fuzzy completos y compactos, respectivamente. 
Teorema 1.2.14. [23] (Teorema de contracción de Banach fuzzy). Sea $(X, M, *)$ un KM-espacio métrico fuzzy tal que

$$
\lim _{t \rightarrow+\infty} M(x, y, t)=1 \text { para cualesquiera } x, y \in X
$$

Sea $T: X \rightarrow X$ una aplicación que satisface

$$
M(T(x), T(y), k t) \geq M(x, y, t) \text { para cualesquiera } x, y \in X, 0<k<1
$$

Entonces la aplicación $T$ posee un único punto fijo.

Teorema 1.2.15. [23] (Teorema de contracción de Edelstein fuzzy). Sea $(X, M, *)$ un KM-espacio métrico fuzzy y sea $T: X \rightarrow X$ una aplicación que satisface

$$
M(T(x), T(y), t)>M(x, y, t) \text { para cualesquiera } t>0, x \neq y
$$

Entonces la aplicación $T$ posee un único punto fijo.

Posteriormente, Subrahmanyam [50] demostró el siguiente teorema, que es una versión fuzzy del teorema de punto fijo de Jungck.

Teorema 1.2.16. [50] Sea $(X, M, *)$ un KM-espacio métrico fuzzy completo y sean $f, g: X \rightarrow X$ dos aplicaciones que satisfacen las siguientes condiciones:

(a) $g(X) \subseteq f(X)$

(b) $f$ es continua

(c) $M(g(x), g(y), k t) \geq M(f(x), f(y), t)$, para cualesquiera $x, y \in X, 0<k<1$.

Entonces $f$ y g poseen un único punto fijo común. 


\subsection{El espacio métrico fuzzy de George y Veeramani}

De ahora en adelante adoptaremos como definición de espacio métrico fuzzy la que sigue.

Definición 1.3.1. Un espacio métrico fuzzy, en el sentido de George y Veeramani [19], es una terna $(X, M, *)$ donde $X$ es un conjunto no vacío, * es una t-norma continua y $M$ es un conjunto fuzzy de $X \times X \times] 0,+\infty[$ (i.e., $M$ es una aplicación de dicho conjunto con valores en el intervalo $[0,1])$ que satisface las siguientes condiciones, para cualesquiera $x, y, z \in X, t, s>0$ :

$(G V 1) M(x, y, t)>0$

(GVR) $M(x, y, t)=1$ si $y$ sólo si $x=y$

$(G V 3) M(x, y, t)=M(y, x, t)$

$(G V 4) M(x, y, t) * M(y, z, s) \leq M(x, z, t+s)$

$(G V 5) M(x, y, \cdot):] 0,+\infty[\longrightarrow[0,1]$ es continua

Si $(X, M, *)$ es un espacio métrico fuzzy, diremos que $(M, *)$ (o $M$, sin más precisión) es una métrica fuzzy en $X$.

Nota 1.3.2. Se podría interpretar a $M(x, y, t)$ como el grado de cercanía entre $x$ e $y$ respecto a $t$, $y$ en tal caso se identificaría a $M(x, y, t)=0$ con $\infty$, y a $M(x, y, t)=1$ con $x=y$.

En ocasiones, sin mención explícita, utilizaremos los siguientes resultados que fueron dados en [19] para un espacio métrico fuzzy $(X, M, *)$, y una $t$-norma continua $*$, respectivamente. 
Proposición 1.3.3. $M(x, y, \cdot)$ es no decreciente, para todo $x, y \in X$.

Por la continuidad de $*$ se puede probar el siguiente resultado.

Proposición 1.3.4. Sea * una t-norma continua, entonces

(i) Dado $r \in] 0,1[$ existe $s \in] 0,1[$ tal que $s * s \geq r$

(ii) Dados $r, s \in] 0,1[$ con $r>s$ existe $t \in] 0,1[$ tal que $r * t \geq s$

Proposición 1.3.5. Sea $(X, d)$ un espacio métrico. Consideremos $a * b=a b$, para cualesquiera $a, b \in[0,1]$, sea $M_{d}$ la función definida en el conjunto $X \times X \times] 0,+\infty[$ de la siguiente manera:

$$
M_{d}(x, y, t)=\frac{t}{t+d(x, y)}
$$

Entonces $\left(X, M_{d}, *\right)$ es un espacio métrico fuzzy.

Definición 1.3.6. La anterior métrica fuzzy $M_{d}$, inducida (generada) por la métrica $d$, se denomina estándar y a $\left(X, M_{d}, *\right)$ se le llama espacio métrico fuzzy estándar (asociado al espacio métrico $(X, d)$ ).

Nota 1.3.7. En el caso en que d sea la métrica euclídea de $\mathbb{R}$, a $M_{d}$ se le denominará métrica fuzzy euclídea de $\mathbb{R}$.

Nota 1.3.8. George y Veeramani [19] demostraron que toda métrica fuzzy $M$ en $X$ genera una topología $\tau_{M}$ en $X$ que tiene como base la familia de bolas abiertas con centro $x$, radio $r$ y parámetro $t$,

$$
\left\{B_{M}(x, r, t): x \in X, 0<r<1, t>0\right\}
$$


donde

$$
B_{M}(x, r, t)=\{y \in X: M(x, y, t)>1-r\}
$$

(Cuando no haya posibilidad de confusión, escribiremos $B(x, r, t)$ en lugar de $\left.B_{M}(x, r, t)\right)$.

Los autores demostraron que $\left(X, \tau_{M}\right)$ es un espacio de Hausdorff que verifica el Primer Axioma de Numerabilidad. Además, en el caso en que $(X, d)$ sea un espacio métrico, entonces la topología que genera la métrica $d$ coincide con la topología $\tau_{M_{d}}$ generada por la métrica fuzzy estándar $M_{d}$.

Definición 1.3.9. [19] Una sucesión $\left\{x_{n}\right\}_{n=1}^{\infty}$ en un espacio métrico fuzzy $(X, M, *)$ se dice que es de Cauchy si para cada $\varepsilon>0$ y $t>0$, existe $n_{0} \in \mathbb{N}$ tal que $M\left(x_{n}, x_{m}, t\right)>1-\varepsilon$, para cualesquiera $n, m \geq n_{0}$.

Un espacio métrico fuzzy $(X, M, *)$ en el que toda sucesión de Cauchy converge se dice que es un espacio métrico fuzzy completo, y en tal caso diremos que $(M, *)$ (o $M$, sin más precisión) es una métrica fuzzy completa en $X$.

Definición 1.3.10. [19] Sea $(M, *)$ una métrica fuzzy en un conjunto $X$. Diremos que $X$ es F-acotado si existen $r \in] 0,1[y t>0$ tal que $M(x, y, t)>$ $1-r$ para cualesquiera $x, y \in X$.

En ocasiones diremos que $M$ es una métrica fuzzy $F$-acotada en $X$.

Proposición 1.3.11. Sea $(X, d)$ un espacio métrico. Entonces:

(i) La topología $\tau_{d}$ generada por la métrica d es la misma que la topología $\tau_{M_{d}}$ generada por su respectiva métrica fuzzy estándar asociada $M_{d}$.

(ii) La sucesión $\left\{x_{n}\right\}_{n=1}^{\infty}$ es una sucesión de d-Cauchy (i.e. es una sucesión de Cauchy en el espacio métrico $(X, d))$ si y sólo si es una sucesión de Cauchy en el espacio métrico fuzzy estándar $\left(X, M_{d}, *\right)$ asociado a d. 
(iii) Un subconjunto $A \subset X$ es acotado en el espacio métrico $(X, d)$ si y sólo si $A$ es $F$-acotado en el espacio métrico fuzzy estándar $\left(X, M_{d}, *\right)$.

Teorema 1.3.12. [19] Una sucesión $\left\{x_{n}\right\}_{n=1}^{\infty}$ en un espacio métrico fuzzy $(X, M, *)$ converge a $x \in X$ si y sólo si $\lim _{n \rightarrow \infty} M\left(x_{n}, x, t\right)=1$.

Es obvio que toda sucesión convergente es de Cauchy.

Proposición 1.3.13. [20] El espacio métrico $(X, d)$ es completo si y sólo si el espacio métrico fuzzy estándar $\left(X, M_{d}, *\right)$ es completo.

Nota 1.3.14. Diremos que un espacio topológico $(X, \tau)$ es fuzzy metrizable si existe una métrica fuzzy $M$ en $X$ tal que $\tau=\tau_{M}$.

Con esta definición se tiene, por la proposición 1.3.5, que todo espacio topológico metrizable es fuzzy metrizable. Además, en [24], los autores probaron que también se verifica el recíproco y, en consecuencia, que la clase de los espacios topológicos metrizables coincide con la clase de los espacios topológicos fuzzy metrizables. Los resultados son los siguientes.

Teorema 1.3.15. [24] Sea $(X, M, *)$ un espacio métrico fuzzy. Entonces $\left(X, \tau_{M}\right)$ es un espacio topológico metrizable.

En la prueba del anterior teorema, los autores demostraron que la familia $\left\{U_{n}: n \in \mathbb{N}\right\}$, donde $U_{n}=\left\{(x, y) \in X \times X: M\left(x, y, \frac{1}{n}\right)>1-\frac{1}{n}\right\}$, es una base (numerable) de una uniformidad $\mathcal{U}_{M}$ en $X$, compatible con la topología deducida de la métrica fuzzy, que, a partir de ahora denominaremos uniformidad deducida de $(M, *)$. Como consecuencia de este resultado se obtiene el siguiente corolario.

Corolario 1.3.16. [24] Un espacio topológico es metrizable si y sólo si es fuzzy metrizable. 
Ello demuestra, como se ha dicho, que la clase de los espacios topológicos metrizables coincide con la clase de los espacios topológicos fuzzy metrizables. Como consecuencia de ello, además, se tiene el siguiente resultado.

Corolario 1.3.17. [20] Todo espacio métrico fuzzy separable verifica el Segundo Axioma de Numerabilidad.

Recordemos que un espacio topológico se dice completamente metrizable si admite una métrica (compatible) completa. Usaremos terminología análoga para un espacio fuzzy metrizable.

Definición 1.3.18. Sea $(X, \tau)$ un espacio topológico. Diremos que $(X, \tau)$ es completamente fuzzy metrizable si admite una métrica fuzzy (compatible) completa.

A continuación enunciaremos resultados obtenidos en relación con el concepto de metrización fuzzy, que son versiones fuzzy de sus análogos en la teoría clásica que pueden encontrarse en [14].

Teorema 1.3.19. [24] Sea $(X, M, *)$ un espacio métrico fuzzy completo. Entonces $\left(X, \tau_{M}\right)$ es completamente metrizable.

Corolario 1.3.20. [24] Un espacio topológico es completamente metrizable si y sólo si es completamente fuzzy metrizable.

Además, es sabido que todo espacio completamente metrizable es de Baire. En consecuencia, por el teorema 1.3.19, se deduce el siguiente corolario.

Corolario 1.3.21. [19] Todo espacio métrico fuzzy completo es de Baire. 


\subsection{Propiedades de los espacios métricos fuzzy}

De ahora en adelante tendremos en consideración las siguientes $t$-normas continuas y que denotaremos por $T_{i}(i=1,2,3)$ :

$$
\begin{aligned}
& T_{1}(x, y)=\min \{x, y\} \\
& T_{2}(x, y)=x y \\
& T_{3}(x, y)=\operatorname{máx}\{0, x+y-1\}
\end{aligned}
$$

Los resultados de la siguiente proposición nos serán de utilidad en lo que sigue.

Proposición 1.4.1. Con las definiciones anteriores se satisfacen las siguientes desigualdades:

(i) $T_{3}(x, y) \leq T_{2}(x, y) \leq T_{1}(x, y)$, para cualesquiera $x, y \in[0,1]$

(ii) $T(x, y) \leq T_{1}(x, y)$ para cualquier -norma $T$ continua y cualesquiera $x, y \in[0,1]$

En ocasiones se utilizará el siguiente lema, aunque no se haga mención explícita.

Lema 1.4.2. Sea $X$ un conjunto arbitrario no vacío. Si $(M, T)$ es una métrica fuzzy en el conjunto $X$ y $T^{\prime}$ es una t-norma continua de manera que $T^{\prime} \leq T$, entonces $\left(M, T^{\prime}\right)$ es una métrica fuzzy en $X$.

Demostración. La única de las propiedades de las métricas fuzzy en la que interviene la $t$-norma continua asociada es la condición $(G V 4)$. Veamos que se cumple para $T^{\prime}$. 
$T^{\prime}(M(x, y, t), M(y, z, s)) \leq T(M(x, y, t), M(y, z, s)) \leq M(x, z, t+s)$

Por tanto $\left(M, T^{\prime}\right)$ es una métrica fuzzy en $X$.

Usando estas propiedades vamos, a continuación, a introducir nuevas formas de construir métricas fuzzy $F$-acotadas a partir de una métrica fuzzy dada.

Proposición 1.4.3. Sea $(X, M, *)$ un espacio métrico fuzzy y sea $k \in] 0,1[$. Definimos:

$$
N(x, y, t)=\operatorname{máx}\{M(x, y, t), k\}, \text { para cualesquiera } x, y \in X, t>0
$$

Entonces el par $(N, *)$ es una métrica fuzzy F-acotada en el conjunto $X$ y genera la misma topología que la métrica fuzzy $M$.

Demostración. Las condiciones $(G V 1),(G V 2),(G V 3)$ y $(G V 5)$ son triviales por la definición de $N$. Veamos que también se cumple la condición $(G V 4)$.

Tenemos que probar que $N(x, y, t) * N(y, z, s) \leq N(x, z, t+s)$, es decir, que

$$
\text { máx }\{M(x, y, t), k\} * \operatorname{máx}\{M(y, z, s), k\} \leq \operatorname{máx}\{M(x, z, t+s), k\}
$$

Distinguimos los siguientes casos.

Primer caso: Si máx $\{M(x, y, t), k\}=M(x, y, t)$, y máx $\{M(y, z, s), k\}=$ $M(y, z, s)$, entonces se tiene que

$$
\begin{gathered}
N(x, y, t) * N(y, z, s)=M(x, y, t) * M(y, z, s) \leq M(x, z, t+s) \leq \\
\leq \operatorname{máx}\{M(x, z, t+s), k\}=N(x, z, t+s)
\end{gathered}
$$

Segundo caso: Si máx $\{M(x, y, t), k\}=k$, máx $\{M(y, z, s), k\}=M(y, z, s)$, entonces

$$
N(x, y, t) * N(y, z, s)=k * M(y, z, s) \leq k \leq
$$




$$
\leq \text { máx }\{M(x, z, t+s), k\}=N(x, z, t+s)
$$

Tercer caso: Si máx $\{M(x, y, t), k\}=k$, máx $\{M(y, z, s), k\}=k$, entonces $N(x, y, t) * N(y, z, s)=k * k \leq k \leq \operatorname{máx}\{M(x, z, t+s), k\}=N(x, z, t+s)$

Todo ello prueba la desigualdad deseada y, en consecuencia $(N, *)$ es una métrica fuzzy, con la que $X$ es $F$-acotado, pues $N(x, y, t) \geq k$ para cualesquiera $x, y \in X, t>0$.

Finalmente, puesto que $B_{M}(x, r, t)=B_{N}(x, r, t)$ para todo $r<1-k$, entonces $\tau_{M}=\tau_{N}$.

Proposición 1.4.4. Sea $i \in\{1,2,3\}$ y sea $k>0$. Sea $\left(X, M, T_{i}\right)$ un espacio métrico fuzzy. Definimos,

$$
N(x, y, t)=\frac{k+M(x, y, t)}{1+k}
$$

para cualesquiera $x, y \in X, t>0$.

Entonces el par $\left(N, T_{i}\right)$ es una métrica fuzzy $F$-acotada en $X$ que, además, genera la misma topología que $M$ para todo $i \in\{1,2,3\}$.

Demostración. Análogamente a la proposición anterior, las condiciones $(G V 1),(G V 2),(G V 3)$ y $(G V 5)$ se cumplen, obviamente, por la propia definición de $N$ cualquiera que sea $T_{i}$. Por tanto, sólo es necesario probar la condición $(G V 4)$ para cada una de las $t$-normas continuas $T_{i}, i=1,2,3$.

Veamos, en primer lugar, la prueba para $T_{1}$.

Se tiene que mín $\{M(x, y, t), M(y, z, s)\} \leq M(x, z, t+s)$, entonces

$$
M(x, y, t) \leq M(x, z, t+s) \text { y } M(y, z, s) \leq M(x, z, t+s) .
$$


Por lo tanto,

$$
\frac{k+M(x, y, t)}{1+k} \leq \frac{k+M(x, z, t+s)}{1+k} \quad \text { y } \quad \frac{k+M(y, z, s)}{1+k} \leq \frac{k+M(x, z, t+s)}{1+k}
$$

En consecuencia, se tiene que

$$
\min \left\{\frac{k+M(x, y, t)}{1+k}, \frac{k+M(y, z, s)}{1+k}\right\} \leq \frac{k+M(x, z, t+s)}{1+k}
$$

La prueba para $T_{2}$ es la siguiente.

Tenemos que verificar que

$$
\frac{k+a}{k+1} \cdot \frac{k+b}{k+1} \leq \frac{k+a b}{k+1}
$$

para cualesquiera $a, b \in] 0,1]$.

La anterior expresión es cierta siempre que

$$
(k+a) \cdot(k+b) \leq(k+1) \cdot(k+a b),
$$

que, a su vez, se cumple si $k \cdot(a+b) \leq k \cdot(a b+1)$, o lo que es lo mismo, si $a+b \leq a b+1$, es decir, si $a(1-b) \leq 1-b$ lo cual es siempre cierto, pues $a \leq 1$.

Veamos la prueba para $T_{3}$.

Es obvio que $N(x, z, t+s)=\frac{k+M(x, z, t+s)}{k+1} \geq 0$

Por otra parte,

$$
\frac{k+M(x, y, t)}{k+1}+\frac{k+M(y, z, s)}{k+1}-1=
$$




$$
=\frac{k+M(x, y, t)+M(y, z, s)-1}{k+1} \leq \frac{k+M(x, z, t+s)}{k+1}=N(x, z, t+s)
$$

En consecuencia, se tiene que

$$
T_{3}(N(x, y, t), N(y, z, s)) \leq N(x, z, t+s)
$$

Claramente, el valor $\frac{k}{1+k}$ es una cota inferior de la métrica fuzzy $N(x, y, t)$ para cualesquiera $x, y \in X, t>0$ y, por lo tanto, $X$ está $F$-acotado para la métrica fuzzy $N$.

Por último, se tiene que para $t>0, r \in] 0,1[$,

$$
\begin{gathered}
B_{M}(x, r, t)=\{y \in X: M(x, y, t)>1-r\}= \\
=\{y \in X:(1+k) N(x, y, t)-k>1-r\}= \\
=\left\{y \in X: N(x, y, t)>1-\frac{r}{1+k}\right\}=B_{N}\left(x, \frac{r}{1+k}, t\right)
\end{gathered}
$$

y también, por otra parte,

$$
\begin{gathered}
B_{N}(x, r, t)=\{y \in X: N(x, y, t)>1-r\}= \\
=\left\{y \in X: \frac{k+M(x, y, t)}{1+k}>1-r\right\}= \\
=\{y \in X: M(x, y, t)>1-(1+k) r\}=B_{M}(x, r(1+k), t)
\end{gathered}
$$

y, por lo tanto, $\tau_{M}=\tau_{N}$

La proposición anterior nos da una forma de construir nuevas métricas fuzzy a partir de una dada, siempre y cuando la $t$-norma asociada a ésta última sea una de las expuestas anteriormente. No obstante, la siguiente cuestión permanece abierta. 
Cuestión abierta 1.1. Sea $(M, *)$ una métrica fuzzy en un conjunto $X y$ sea $k>0$. ¿Es el par $\left(\frac{k+M(x, y, t)}{1+k}, *\right)$ una métrica fuzzy en $X$ ?

Las anteriores proposiciones nos dan métodos para construir una nueva métrica fuzzy a partir de una métrica fuzzy dada. A continuación, las siguientes proposiciones, nos dan la forma de conseguir dos nuevas métricas a partir de dos dadas.

Proposición 1.4.5. Sean $\left(M_{1}, *\right)$ y $\left(M_{2}, *\right)$ dos métricas fuzzy en un conjunto arbitrario $X$ tales que $a * b \neq 0$ para cualesquiera $a, b \neq 0$. Entonces el par $(N, *)$ es una métrica fuzzy en $X$, donde:

$$
N(x, y, t)=M_{1}(x, y, t) * M_{2}(x, y, t)
$$

Demostración. $N(x, y, t)>0$ puesto que $M_{1}(x, y, t)>0$ y $M_{2}(x, y, t)>0$ por ser, ambas, métricas fuzzy.

La simetría de las métricas fuzzy $M_{1}$ y $M_{2}$ junto con la conmutatividad de la $t$-norma continua $*$ nos prueba la simetría de $N$.

Por otra parte, resulta obvio que, si $x=y$, entonces $N(x, y, t)=M_{1}(x, y, t) * M_{2}(x, y, t)=1 * 1=1$, y, recíprocamente, si $N(x, y, t)=1$ se tiene que $1=M_{1}(x, y, t) * M_{2}(x, y, t)$ y ello sólamente es posible si $M_{1}(x, y, t)=M_{2}(x, y, t)=1 \mathrm{y}$, en consecuencia, $x=y$.

Veamos la desigualdad triangular.

$$
\begin{gathered}
N(x, y, t) * N(y, z, s)=M_{1}(x, y, t) * M_{2}(x, y, t) * M_{1}(y, z, s) * M_{2}(y, z, s)= \\
=M_{1}(x, y, t) * M_{1}(y, z, s) * M_{2}(x, y, t) * M_{2}(y, z, s) \leq \\
\leq M_{1}(x, z, t+s) * M_{2}(x, z, t+s)=N(x, z, t+s)
\end{gathered}
$$


Veamos la continuidad de la aplicación $N(x, y, \cdot)$. Fijemos $x, y \in X$. Sean $t_{0}>0$ y $\varepsilon>0$, y supongamos que $\left.N\left(x, y, t_{0}\right) \in\right] 0,1[$. Por la continuidad de $*$, existe $r \in] 0,1\left[\right.$ que podemos elegir de manera que $0<N\left(x, y, t_{0}\right)-r<$ $N\left(x, y, t_{0}\right)+r \leq 1$, y que verifica

$$
\begin{gathered}
] M_{1}\left(x, y, t_{0}\right)-r, M_{1}\left(x, y, t_{0}\right)+r[*] M_{2}\left(x, y, t_{0}\right)-r, M_{2}\left(x, y, t_{0}\right)+r[\subset \\
\subset] N\left(x, y, t_{0}\right)-\varepsilon, N\left(x, y, t_{0}\right)+\varepsilon[
\end{gathered}
$$

Por la continuidad de $M_{i}(x, y, \cdot)(i=1,2)$, podemos elegir $\delta_{i}>0$ de manera que si $\left|t-t_{0}\right|<\delta_{i}$, entonces $\left|M_{i}\left(x, y, t_{0}\right)-M_{i}(x, y, t)\right|<r$ y, por tanto, para $\delta=\operatorname{mín}\left\{\delta_{1}, \delta_{2}\right\}$, se tiene que si $\left|t-t_{0}\right|<\delta$, entonces

$$
\left.M_{1}(x, y, t) * M_{2}(x, y, t)=N(x, y, t) \in\right] N\left(x, y, t_{0}\right)-\varepsilon, N\left(x, y, t_{0}\right)+\varepsilon[
$$

Si $N\left(x, y, t_{0}\right)=1$, se puede hacer un razonamiento similar.

Nota 1.4.6. Si existen $a, b \neq 0$ tales que $a * b=0$, entonces el par $(N, *)$ no es, necesariamente, una métrica fuzzy en $X$, como prueba el siguiente contraejemplo.

Contraejemplo 1.4.7. Sean $M_{1}(x, y, t)=M_{2}(x, y, t)=\frac{t}{t+|x-y|}$ las métricas fuzzy estándar definidas en $\mathbb{R}$.

Sea $x * y=T_{3}(x, y)=\operatorname{máx}\{0, x+y-1\}$.

Sabemos, por el lema 1.4.2, que $\left(M_{1}, T_{3}\right)$ y $\left(M_{2}, T_{3}\right)$ son métricas fuzzy en $\mathbb{R}$. Veamos que $M_{1} * M_{2}$ no es una métrica fuzzy en $\mathbb{R}$. En efecto, se tiene:

$$
M(1,3,1)=M_{1}(1,3,1) * M_{2}(1,3,1)=\frac{1}{1+2} * \frac{1}{1+2}=\frac{1}{3} * \frac{1}{3}=0
$$

que contradice la propiedad (GV1) de las métricas fuzzy. 
Nota 1.4.8. Como una obvia generalización de la proposición anterior, es fácil deducir que si $\left(M_{1}, *\right)$ y $\left(M_{2}, *\right)$ son métricas fuzzy en $X$ e $Y$ respectivamente, entonces $P\left(\left(x_{1}, y_{1}\right),\left(x_{2}, y_{2}\right), t\right)=M_{1}\left(x_{1}, x_{2}, t\right) * M_{2}\left(y_{1}, y_{2}, t\right)$ es una métrica fuzzy en $X \times Y$ siempre que $x * y>0$ cuando $x, y \neq 0$. (El contraejemplo 1.4 .7 prueba que la condición $x * y>0$ si $x, y \neq 0$ no puede suprimirse, con lo que el enunciado de [20] no es correcto).

Proposición 1.4.9. Sean $\left(M_{1}, *\right)$ y $\left(M_{2}, *\right)$ dos métricas fuzzy en un conjunto arbitrario $X$. Entonces el par $(M, *)$ es una métrica fuzzy en $X$, donde

$$
M(x, y, t)=\min \left\{M_{1}(x, y, t), M_{2}(x, y, t)\right\}
$$

Demostración. Es obvio que $M(x, y, t)>0$ para cualesquiera $x, y \in$ $X, t>0$.

Por otra parte, la simetría de la métrica $M$ también resulta evidente, en virtud de la simetría de $M_{1}$ y $M_{2}$.

Además, $M(x, y, t)=1$ si y sólo si $x=y$ por ser $M_{1}$ y $M_{2}$ métricas fuzzy.

Veamos que también se cumple la desigualdad triangular.

$$
\begin{gathered}
M(x, y, t) * M(y, z, s)= \\
=\operatorname{mín}\left\{M_{1}(x, y, t), M_{2}(x, y, t)\right\} * \min \left\{M_{1}(y, z, s) * M_{2}(y, z, s)\right\} \leq \\
\leq \min \left\{M_{1}(x, y, t) * M_{1}(y, z, s), M_{2}(x, y, t) * M_{2}(y, z, s)\right\} \leq \\
\leq \min \left\{M_{1}(x, z, t+s), M_{2}(x, z, t+s)\right\}=M(x, z, t+s)
\end{gathered}
$$

Por último, como el mínimo de funciones continuas es continua, se deduce la continuidad de la aplicación $M(x, y, \cdot):] 0,+\infty[\rightarrow] 0,1]$.

Proposición 1.4.10. Sean $\left(M_{1}, *\right)$ y $\left(M_{2}, *\right)$ dos métricas fuzzy en un conjunto arbitrario $X$ tales que $a * b \neq 0$ para cualesquiera $a, b \neq 0$. Entonces las 
topologías $\tau_{M}$ y $\tau_{N}$ generadas por las métricas fuzzy $M$ y $N$, respectivamente, de las proposiciones anteriores, son idénticas.

Demostración. Veamos que $B_{N}(x, r, t) \subseteq B_{M}(x, r, t)$

Sea $y \in B_{N}(x, r, t)$, entonces

$$
\min \left\{M_{1}(x, y, t), M_{2}(x, y, t)\right\} \geq M_{1}(x, y, t) * M_{2}(x, y, t)>1-r
$$

$\mathrm{y}$, por lo tanto, $y \in B_{M}(x, r, t)$.

Elijamos $r, s \in] 0,1[$ de manera que $(1-r) *(1-r)>1-s$. Veamos que $B_{M}(x, r, t) \subseteq B_{N}(x, s, t)$.

Sea $y \in B_{M}(x, r, t)$, entonces $\min \left\{M_{1}(x, y, t), M_{2}(x, y, t)\right\}>1-r$. En consecuencia, $M_{1}(x, y, t)>(1-r)$ y $M_{2}(x, y, t)>1-r$. Por lo tanto $N(x, y, t)=M_{1}(x, y, t) * M_{2}(x, y, t)>(1-r) *(1-r)>1-s$ y podemos concluir que $y \in B_{N}(x, s, t)$. Todo ello prueba que $\tau_{M}=\tau_{N}$.

Corolario 1.4.11. Si $\left(M_{1}, T_{2}\right)$ y $\left(M_{2}, T_{2}\right)$ son dos métricas fuzzy estándar, deducidas de las métricas $d_{1}$ y $d_{2}$ respectivamente, por la proposición anterior se tiene que tanto $M=\operatorname{mín}\left\{M_{1}, M_{2}\right\}$ como $N=M_{1} \cdot M_{2}$ son métricas fuzzy. Ahora bien $M$ es una métrica fuzzy estándar mientras que $N$ no lo es.

Demostración. $M(x, y, t)=\operatorname{mín}\left\{M_{1}(x, y, t), M_{2}(x, y, t)\right\}=$

$$
=\min \left\{\frac{t}{t+d_{1}(x, y)}, \frac{t}{t+d_{2}(x, y)}\right\}=\frac{t}{t+\operatorname{máx}\left\{d_{1}(x, y), d_{2}(x, y)\right\}}
$$

que es la métrica fuzzy estándar asociada a la métrica

$$
d(x, y)=\operatorname{máx}\left\{d_{1}(x, y), d_{2}(x, y)\right\}
$$


Por otra parte, sean $d_{1}(x, y)=|x-y|$ y $d_{2}(x, y)=\operatorname{máx}\{x, y\}$ métricas en $\mathbb{R}$, y sean $M_{1}(x, y, t)$ y $M_{2}(x, y, t)$ las métricas fuzzy estándar asociadas a la métricas $d_{1}$ y $d_{2}$, respectivamente.

Sea $N(x, y, t)=M_{1}(x, y, t) \cdot M_{2}(x, y, t)$. Veamos que $N$ no es una métrica fuzzy estándar.

$$
\begin{gathered}
M(x, y, t)=\frac{t}{t+d_{1}(x, y)} \cdot \frac{t}{t+d_{2}(x, y)}= \\
=\frac{t^{2}}{t^{2}+t \cdot d_{1}(x, y)+t \cdot d_{2}(x, y)+d_{1}(x, y) \cdot d_{2}(x, y)}= \\
=\frac{t}{t+\left[d_{1}(x, y)+d_{2}(x, y)+\frac{d_{1}(x, y) \cdot d_{2}(x, y)}{t}\right]}
\end{gathered}
$$

Sea ahora $D(x, y)=d_{1}(x, y)+d_{2}(x, y)+\frac{d_{1}(x, y) \cdot d_{2}(x, y)}{t}$

Veamos que $D(x, y)$ no es una métrica.

$$
\begin{gathered}
D(1,2)+D(2,3)= \\
d_{1}(1,2)+d_{2}(1,2)+\frac{d_{1}(1,2) \cdot d_{2}(1,2)}{t}+d_{1}(2,3)+d_{2}(2,3)+\frac{d_{1}(2,3) \cdot d_{2}(2,3)}{t}= \\
=1+2+\frac{1 \cdot 2}{t}+1+3+\frac{1 \cdot 3}{t}=7+\frac{5}{t}
\end{gathered}
$$

Por otra parte,

$$
\begin{gathered}
D(1,3)=d_{1}(1,3)+d_{2}(1,3)+\frac{d_{1}(1,3) \cdot d_{2}(1,3)}{t}= \\
=2+3+\frac{2 \cdot 3}{t}=5+\frac{6}{t}
\end{gathered}
$$


Si $t<\frac{1}{2}$, entonces se tiene que $7+\frac{5}{t}<5+\frac{6}{t}$ y, en consecuencia, $D(1,2)+D(2,3)<D(1,3)$, que prueba que $D$ no es una métrica en $\mathbb{R}$.

Podemos concluir, por tanto, que $N$ no es una métrica fuzzy estándar.

Definición 1.4.12. [24] Un espacio métrico fuzzy $(X, M, *)$ es precompacto si para cualesquiera $r \in] 0,1[, t>0$ existe un subconjunto finito $A$ de $X$ tal que

$$
X=\cup\{B(a, r, t): a \in A\}
$$

En este caso se dice que $M$ es una métrica fuzzy precompacta en $X$.

Definición 1.4.13. [24] Sea $(X, M, *)$ un espacio métrico fuzzy. Diremos que $(X, M, *)$ es compacto si $\left(X, \tau_{M}\right)$ es un espacio topológico compacto.

El siguiente resultado caracteriza los espacios métricos fuzzy separables en términos de métricas fuzzy precompactas.

Teorema 1.4.14. [24] Un espacio métrico fuzzy $(X, M, *)$ es separable si $y$ sólo si $\left(X, \tau_{M}\right)$ admite una métrica fuzzy compatible precompacta.

En [24] también se obtuvieron las siguientes caracterizaciones de la compacidad de espacios métricos fuzzy semejantes al caso clásico.

Teorema 1.4.15. [24] Un espacio métrico fuzzy es compacto si y sólo si es precompacto y completo.

Teorema 1.4.16. [24] Un espacio topológico metrizable es compacto si y sólo si toda métrica fuzzy compatible es precompacta.

Teorema 1.4.17. [24] Un espacio topológico metrizable es compacto si y sólo si toda métrica fuzzy compatible es completa. 
Con la anterior definición, Gregori y Romaguera [24], probaron que una métrica fuzzy es precompacta si y sólo si toda sucesión contiene una subsucesión de Cauchy. Con ello, se puede probar la siguiente proposición, que relaciona la precompacidad de una métrica con la precompacidad de su métrica fuzzy estándar asociada.

Proposición 1.4.18. Sea $(X, d)$ un espacio métrico y sea $M_{d}$ la métrica fuzzy estándar asociada a d. Entonces, d es una métrica precompacta si y sólo si $M_{d}$ es una métrica fuzzy precompacta.

Demostración. Supongamos que $d$ es una métrica precompacta. Sea $\left\{x_{n}\right\}_{n=1}^{\infty}$ una sucesión en $X$. Por ser $d$ precompacta, $\left\{x_{n}\right\}_{n=1}^{\infty}$ tiene una subsucesión de Cauchy $\left\{x_{k(n)}\right\}_{n=1}^{\infty}$ en $(X, d)$.

Dados $r \in] 0,1\left[\mathrm{y} t>0\right.$, tomamos $\varepsilon=\frac{t}{1-r}-t$. Entonces existe $n_{0} \in \mathbb{N}$ tal que $d\left(x_{k(n)}, x_{k(m)}\right)<\varepsilon$ para cualesquiera $n, m \geq n_{0}$.

En consecuencia, $M_{d}\left(x_{k(n)}, x_{k(m)}, t\right)>\frac{t}{t+\varepsilon}=1-r$, para $n, m \geq n_{0}$. Por lo tanto, $\left\{x_{k(n)}\right\}_{n=1}^{\infty}$ es una sucesión de Cauchy en $\left(X, M_{d}, *\right)$ y, por el lema 3 de [24], $M_{d}$ es una métrica fuzzy precompacta.

Recíprocamente, supongamos que $M_{d}$ es precompacta. Sea $\left\{x_{n}\right\}_{n=1}^{\infty}$ una sucesión en $X$. Por el mencionado lema de [24], existe una subsucesión de Cauchy $\left\{x_{k(n)}\right\}_{n=1}^{\infty}$ en $\left(X, M_{d}, *\right)$. Dado $\left.\varepsilon \in\right] 0, \frac{1}{2}\left[\right.$, existe $n_{0} \in \mathbb{N}$ tal que $\left\{x_{k(n)}, x_{k(m)}, \frac{1}{2}\right)>1-\varepsilon$, para cualesquiera $n, m \geq n_{0}$. Entonces se tiene que

$$
\frac{\frac{1}{2}}{\frac{1}{2}+d\left(x_{k(n)}, x_{k(m)}\right)}>1-\varepsilon,
$$

es decir, $d\left(x_{k(n)}, x_{k(m)}\right)<\frac{\varepsilon}{2(1-\varepsilon)}<\varepsilon$. Por lo tanto $\left\{x_{n}\right\}_{n=1}^{\infty}$ es una sucesión de Cauchy en $(X, d)$ y, en consecuencia, $d$ es precompacta. 
Proposición 1.4.19. Sea $(X, M, *)$ un espacio métrico fuzzy precompacto. Supongamos que $a * b \neq 0$ para cualesquiera $a, b \neq 0$. Entonces, $(X, M, *)$ es un espacio métrico fuzzy F-acotado.

Demostración. Sea $(X, M, *)$ un espacio métrico fuzzy precompacto. Fijemos $r \in] 0,1\left[, t>0\right.$. Entonces, existe $\left\{a_{1}, a_{2}, \ldots, a_{n}\right\} \subset X$ tal que $X=$ $\bigcup_{i=1}^{n} B\left(a_{i}, r, t\right)$.

Sean $x, y \in X$, entonces $x \in B\left(a_{i}, r, t\right)$ e $y \in B\left(a_{j}, r, t\right)$ para algún $1 \leq$ $i, j \leq n$. Sea ahora $\alpha=\min \left\{M\left(a_{i}, a_{j}, t\right): 1 \leq i, j \leq n\right\}>0$. Con ello, se tiene que

$$
\begin{gathered}
M(x, y, t) \geq M\left(x, a_{i}, \frac{t}{3}\right) * M\left(a_{i}, a_{j}, \frac{t}{3}\right) * M\left(a_{j}, y, \frac{t}{3}\right)> \\
>(1-r) * \alpha *(1-r)
\end{gathered}
$$

Por hipótesis, existe $s \in] 0,1[$ tal que $(1-r) *(1-r) * \alpha>(1-s)$, por lo tanto $M(x, y, t)>(1-s) \mathrm{y}$, en consecuencia, $M$ es una métrica fuzzy F-acotada.

La siguiente es una cuestión abierta.

Cuestión abierta 1.2. ¿Todo espacio métrico fuzzy precompacto es Facotado?

Nota 1.4.20. La implicación recíproca de la anterior proposición no es cierta en general. De hecho, el subespacio $X$ del espacio métrico de Hilbert $\left(\mathbb{R}^{\infty}, d\right)$, formado por los puntos de peso unidad $(0, \ldots, 0,1,0, \ldots)$, no es precompacto pero sí que es acotado (tiene diámetro $\sqrt{2}$ ) mientras que el espacio métrico fuzzy $\left(X, M_{d}, *\right)$ es $F$-acotado pero la métrica $M_{d}$ no es precompacta, como prueban las proposiciones 1.3 .11 y 1.4.18, respectivamente. 


\subsection{Ejemplos de espacios métricos fuzzy}

A lo largo de la presente sección vamos a mostrar diversos ejemplos de espacios métricos fuzzy cuyas $t$-normas continuas asociadas son $T_{i}(i=2,3)$, pero que no son espacios métricos fuzzy si su $t$-norma asociada es $T_{i-1}$. En primer lugar, para poder llevar a cabo este objetivo, necesitaremos el siguiente lema:

Lema 1.5.1. Sea $(X, d)$ un espacio métrico y sean $s, t>0$. Entonces se satisface la siguiente desigualdad para cualquier $n \geq 1$ :

$$
\frac{d(x, z)}{(t+s)^{n}} \leq \operatorname{máx}\left\{\frac{d(x, y)}{t^{n}}, \frac{d(y, z)}{s^{n}}\right\}
$$

Demostración. Vamos a distinguir tres casos.

(1) $d(x, z) \leq d(x, y)$

(2) $d(x, z) \leq d(y, z)$

(3) $d(x, z) \geq d(x, y)$ y $d(x, z) \geq d(y, z)$

En los casos (1) y (2), la desigualdad resulta evidente.

Supongamos que se cumple el tercero de los casos. Distinguimos dos posibilidades.

$$
\begin{aligned}
& (3,1) \quad d(x, z)=d(x, y)+d(y, z) \\
& (3,2) \quad d(x, z) \leq d(x, y)+d(y, z)
\end{aligned}
$$

Supongamos que se satisface $(3,1)$. Entonces, podemos tomar $\beta \in] 0,1[$ tal que $d(x, y)=\beta d(x, z), \mathrm{y}$, en consecuencia, $d(y, z)=(1-\beta) d(x, z)$

Para demostrar la desigualdad deseada tendremos que probar que

$$
\frac{1}{(t+s)^{n}} \leq \operatorname{máx}\left\{\frac{\beta}{t^{n}}, \frac{1-\beta}{s^{n}}\right\}
$$


Consideremos las siguientes funciones:

$$
\begin{aligned}
& f(\beta)=\frac{t^{n}}{\beta} \\
& g(\beta)=\frac{s^{n}}{1-\beta}
\end{aligned}
$$

Entonces, $f$ es estrictamente decreciente y $g$ es estrictamente creciente.

El valor máximo de mín $\left\{\frac{t^{n}}{\beta}, \frac{s^{n}}{1-\beta}\right\}$ se alcanza cuando $f(\beta)=g(\beta)$ y ello se da cuando $\beta=\frac{t^{n}}{t^{n}+s^{n}}$. Entonces,

$$
(t+s)^{n} \geq t^{n}+s^{n}=f\left(\frac{t^{n}}{t^{n}+s^{n}}\right) \geq \operatorname{mín}\left\{\frac{t^{n}}{\beta}, \frac{s^{n}}{1-\beta}\right\}
$$

y, por lo tanto,

$$
\frac{1}{(t+s)^{n}} \leq \operatorname{máx}\left\{\frac{\beta}{t^{n}}, \frac{1-\beta}{s^{n}}\right\}
$$

La posibilidad $(3,2)$ es consecuencia inmediata del caso anterior.

Nota 1.5.2. La restricción $n \geq 1$ en el anterior lema es necesaria como prueba el siguiente contraejemplo.

Sea $(X, d)$ la recta con la métrica usual y tomemos $n=\frac{1}{2}, t=3, s=1$, $x=1, y=3, z=\frac{1}{2}$. Entonces es fácil verificar que la desigualdad del anterior lema no se satisface.

Ejemplo 1.5.3. Sea $(X, d)$ un espacio métrico y sea $B(x, r)$ la bola abierta de centro $x \in X$ y radio $r>0$. Entonces:

Para todo $n \geq 1,\left(X, M, T_{1}\right)$ es un espacio métrico fuzzy donde $M$ viene dada por la expresión

$$
M(x, y, t)=\frac{1}{e^{\frac{d(x, y)}{t^{n}}}}, \text { para todo } x, y \in X, t>0
$$


Además, se tiene que $\tau_{M}=\tau_{d}$.

(Este ejemplo es una generalización del ejemplo 2.7 de George y Veeramani [19])

Demostración. La propia definición de $M$ hace evidente el hecho de que $M(x, y, t)>0$ para cualesquiera $x, y \in X, t>0$.

La simetría de $M$ es consecuencia de la simetría de la métrica $d$.

Si $M(x, y, t)=1$, entonces $d(x, y)=0$ y ello implica que $x=y$. Por otra parte, si $x=y$, entonces $d(x, y)=0$ y, por lo tanto, $M(x, y, t)=1$ para todo $t>0$.

La desigualdad triangular es una consecuencia del lema anterior.

La continuidad de la función $M(x, y, \cdot)$ es evidente.

Por último,

$$
\begin{gathered}
B_{M}(x, r, t)=\{y \in X: M(x, y, t)>1-r\}=\left\{y \in X: e^{\frac{d(x, y)}{t^{n}}}<\frac{1}{1-r}\right\}= \\
=\left\{y \in X: \frac{d(x, y)}{t^{n}}<-\ln (1-r)\right\}= \\
=\left\{y \in X: d(x, y)<-t^{n} \ln (1-r)\right\}=B\left(x,-t^{n} \ln (1-r)\right)
\end{gathered}
$$

$y$, por otra parte, para $t>0$, se tiene

$$
\begin{gathered}
B(x, r)=\{y \in X: d(x, y)<r\}= \\
=\left\{y \in X: \frac{d(x, y)}{t^{n}}<\frac{r}{t^{n}}\right\}=\left\{y \in X: e^{\frac{d(x, y)}{t^{n}}}<e^{\frac{r}{t^{n}}}\right\}=
\end{gathered}
$$




$$
=\left\{y \in X: \frac{1}{e^{\frac{d(x, y)}{t^{n}}}}>1-\frac{1-e^{\frac{r}{t^{n}}}}{e^{\frac{r}{t^{n}}}}\right\}=B_{M}\left(x, 1-\frac{1}{e^{\frac{r}{t^{n}}}}, t\right)
$$

Por tanto, $\tau_{M}=\tau_{d}$.

Nota 1.5.4. El anterior ejemplo no se puede generalizar para $n<1$. En efecto, si consideramos la métrica usual en $\mathbb{R}$, se tiene, para $n=\frac{1}{2}, x=1$, $y=2, z=3$, que

$$
M(x, z, 2)=\frac{1}{e^{\frac{2}{\sqrt{2}}}}<\operatorname{mín}\left\{\frac{1}{e}, \frac{1}{e}\right\}=\operatorname{mín}\{M(x, y, 1), M(y, z, 1)\}
$$

Sin embargo, para la $t$-norma $T_{2}$ (producto usual), $M$ sí resulta ser una métrica fuzzy para $n \geq 0$.

En efecto, de $d(x, z) \leq \frac{(t+s)^{n}}{t^{n}} d(x, y)+\frac{(t+s)^{n}}{s^{n}} d(y, z)$ se tiene que $\frac{d(x, z)}{(t+s)^{n}} \leq \frac{d(x, y)}{t^{n}}+\frac{d(y, z)}{s^{n}}$ de lo que se desprende fácilmente la desigualdad $M(x, z, t+s) \geq M(x, y, t) \cdot M(y, z, s)$.

Ejemplo 1.5.5. Sea $(X, d)$ un espacio métrico y sea $B(x, r)$ la bola abierta de centro $x \in X$ y radio $r>0$. Entonces:

Para cualesquiera $k, m \in \mathbb{R}^{+}$y $n \geq 1,\left(X, M, T_{1}\right)$ es un espacio métrico fuzzy donde $M$ viene dada por la expresión

$$
M(x, y, t)=\frac{k t^{n}}{k t^{n}+m d(x, y)}, \text { para todo } x, y \in X, t>0
$$

Además, se tiene que $\tau_{M}=\tau_{d}$.

Demostración. Por el lema anterior, se tiene que 


$$
1+\frac{m d(x, z)}{k(t+s)^{n}} \leq \operatorname{máx}\left\{1+\frac{m d(x, y)}{k t^{n}}, 1+\frac{m d(y, z)}{k s^{n}}\right\}
$$

en consecuencia,

$$
\frac{k(t+s)^{n}}{k(t+s)^{n}+m d(x, z)} \geq \operatorname{mín}\left\{\frac{k t^{n}}{k t^{n}+m d(x, y)}, \frac{k s^{n}}{k s^{n}+m d(y, z)}\right\}
$$

y la desigualdad triangular se satisface.

Por último, para cualesquiera $x \in X, t>0$ y $r \in] 0,1[$ se tiene que

$$
\begin{aligned}
& B_{M}(x, r, t)=\left\{y \in X: \frac{k t^{n}}{k t^{n}+m d(x, y)}>1-r\right\}= \\
= & \left\{y \in X: d(x, y)<\frac{k t^{n} r}{m(1-r)}\right\}=B\left(x, \frac{k t^{n} r}{m(1-r)}\right) .
\end{aligned}
$$

Por otra parte,

$$
B(x, r)=B_{M}\left(x, \frac{m r}{k t^{n}+m r}, t\right)
$$

y, en consecuencia, se tiene que $\tau_{M}=\tau_{d}$.

Nota 1.5.6. En [19, Remark 2.10] se asegura que la anterior métrica fuzzy lo es para $n \in \mathbb{R}^{+}$, pero ello no es cierto. En efecto, si consideramos en $\mathbb{R}$ la métrica usual, y elegimos $n=\frac{1}{2}, k=m=1$, entonces, para $x=1, y=2$, $z=3$, se tiene que $M(x, z, 2)=\frac{\sqrt{2}}{\sqrt{2}+2}<\min \{M(x, y, 1), M(y, z, 1)\}$.

Por otra parte, en el ejemplo 2.9 de [19] se afirma que la anterior métrica fuzzy lo es para $k, m, n \in \mathbb{R}^{+}$si consideramos la $t$-norma producto $T_{2}$, lo cual es fácil de verificar. Más todavía, se verifica también para $n=0$. En efecto,

$$
\frac{k}{k+m d(x, y)} \cdot \frac{k}{m d(y, z)}=\frac{k}{k+m(d(x, y)+d(y, z))+\frac{m^{2}}{k} d(x, y) d(y, z)} \leq
$$




$$
\leq \frac{k}{k+m(d(x, y)+d(y, z))} \leq \frac{k}{k+m d(x, z)}
$$

Ejemplo 1.5.7. Sea $X$ el intervalo $] 0,+\infty[$ de la recta real y sea $a>0$. Entonces $\left(X, M, T_{2}\right)$ es un espacio métrico fuzzy, pero $\left(X, M, T_{1}\right)$ no lo es, donde $M$ viene dada por la expresión:

$$
M(x, y, t)=\left\{\begin{array}{l}
\left(\frac{x}{y}\right)^{a} \quad \text { si } x \leq y \\
\left(\frac{y}{x}\right)^{a} \quad \text { si } y \leq x
\end{array} \text {, para cualesquiera } x, y \in X, t>0\right.
$$

(Cabe destacar que este ejemplo es una generalización del introducido por George y Veeramani [19]. En aquel caso $X=\mathbb{N}$ y $a=1$ ).

Demostración. Es obvio que $M(x, y, t) \geq 0$ por ser $x, y, a>0$. Además, la simetría resulta evidente.

Por otra parte, $M(x, y, t)=1$ si sólo si $\left(\frac{x}{y}\right)^{a}=1$ ó $\left(\frac{y}{x}\right)^{a}=1$, lo cual es cierto si y sólo si $x=y$.

Veamos que también se cumple la desigualdad triangular. Distinguiremos varios casos.

1. Si $x \leq y \leq z$, entonces

$$
M(x, y, t) \cdot M(y, z, s)=\left(\frac{x}{y}\right)^{a} \cdot\left(\frac{y}{z}\right)^{a}=\left(\frac{x}{z}\right)^{a}=M(x, z, t+s)
$$

2. Si $x \leq z \leq y$, entonces

$$
M(x, y, t) \cdot M(y, z, s)=\left(\frac{x}{y}\right)^{a} \cdot\left(\frac{z}{y}\right)^{a} \leq\left(\frac{x z}{z z}\right)^{a}=\left(\frac{x}{z}\right)^{a}=M(x, z, t+s)
$$


3. Los casos $y \leq x \leq z$ y $y \leq z \leq x$ son análogos a los dos anteriores cambiando $x$ por $y$.

4. Si $z \leq x \leq y$, entonces

$$
M(x, y, t) \cdot M(y, z, s)=\left(\frac{x}{y}\right)^{a} \cdot\left(\frac{z}{y}\right)^{a} \leq\left(\frac{x z}{x x}\right)^{a}=\left(\frac{x}{z}\right)^{a}=M(x, z, t+s)
$$

5. El caso en que $z \leq y \leq x$ es análogo al anterior.

Esto completa la demostración.

Nota 1.5.8. En el ejemplo anterior, si tomamos $x \in X, t>0, y \quad r \in] 0,1[$, se tiene que

$$
\begin{gathered}
B(x, r, t)=\left\{y \in X: x \leq y,\left(\frac{x}{y}\right)^{a}>1-r\right\} \cup\left\{y \in X: y \leq x,\left(\frac{y}{x}\right)^{a}>1-r\right\}= \\
=\left\{y \in X: x \leq y<\frac{x}{(1-r)^{\frac{1}{a}}}\right\} \cup\left\{y \in X: x(1-r)^{\frac{1}{a}}<y \leq x\right\}= \\
=] x(1-r)^{\frac{1}{a}}, \frac{x}{(1-r)^{\frac{1}{a}}}[
\end{gathered}
$$

En consecuencia, $B(x, r, t)$ es un intervalo abierto de $\mathbb{R}$ cuyo diámetro converge a 0 cuando $r$ tiende a 0 . Por ello, $\tau_{M}$ es la topología usual de $\mathbb{R}$ relativa al conjunto $X$.

Por otra parte, se tiene que $\left(X, M, T_{1}\right)$ no es un espacio métrico fuzzy, como prueba el siguiente contrajemplo.

Contraejemplo 1.5.9. Sean, $a=1, x=1, y=2 y z=3$. Entonces

$$
M(x, z, t+s)=\frac{1}{3}<\min \left\{\frac{1}{2}, \frac{2}{3}\right\}=\min \{M(x, y, t), M(y, z, s)\} .
$$


Ejemplo 1.5.10. Sea $X$ el intervalo real $] 1,+\infty[y$ consideremos la aplicación $\left.\left.M: X^{2} \times\right] 0+\infty[\longrightarrow] 0,1\right]$ dada por la siguiente expresión

$$
M(a, b, t)=1-\left(\frac{1}{a \wedge b}-\frac{1}{a \vee b}\right), \text { para cualesquiera } a, b \in X, t>0 .
$$

Entonces $\left(X, M, T_{3}\right)$ es un espacio métrico fuzzy, mientras que $\left(X, M, T_{2}\right)$ no lo es.

Además, la topología $\tau_{M}$ generada por la métrica fuzzy $M$ en el conjunto $X$ coincide con la topología usual de $\mathbb{R}$ relativa a $X$.

Demostración. Para cualesquiera $a, b \in] 1,+\infty\left[\right.$ se tiene que $\frac{1}{a \wedge b}<1 \mathrm{y}$ que $\frac{1}{a \vee b}<1$. Además, $\frac{1}{a \wedge b} \geq \frac{1}{a \vee b}$ y, por lo tanto, $M(a, b, t)>0$.

La simetría de $M$ resulta evidente por la propia definición.

$M(a, b, t)=1$ si y sólo si $\left(\frac{1}{a \wedge b}-\frac{1}{a \vee b}\right)=0$, lo cual es cierto si y sólo si $a \wedge b=a \vee b$, que ocurre si y sólo si $a=b$.

Veamos que también se cumple la desigualdad triangular, que tiene la siguiente expresión (para la $t$-norma $T_{3}$ )

$\left[1-\left(\frac{1}{a \wedge b}-\frac{1}{a \vee b}\right)\right]+\left[1-\left(\frac{1}{b \wedge c}-\frac{1}{b \vee c}\right)\right]-1 \leq 1-\left(\frac{1}{a \wedge c}-\frac{1}{a \vee c}\right)$

Si $a=b$ ó $a=c$ ó $b=c$, esta desigualdad resulta obvia.

Si $a \neq b \neq c \neq a$ distinguiremos 6 casos.

(1) Supongamos que $a<b<c$. En este caso, la desigualdad resulta una igualdad. 
(2) Si $a<c<b$ la desigualdad resulta $\frac{1}{b}+\frac{1}{b}+\frac{1}{a} \leq \frac{1}{a}+\frac{1}{c}+\frac{1}{c}$ que es cierta, por ser $\frac{1}{b}<\frac{1}{c}$.

(3) Si $c<a<b$ la desigualdad es $\frac{1}{b}+\frac{1}{b}+\frac{1}{c} \leq \frac{1}{a}+\frac{1}{c}+\frac{1}{a}$ que es cierta, por ser $\frac{1}{b}<\frac{1}{a}$.

(4) Si $b<a<c$ la desigualdad es $\frac{1}{a}+\frac{1}{c}+\frac{1}{a} \leq \frac{1}{b}+\frac{1}{b}+\frac{1}{c}$ que es cierta, por ser $\frac{1}{a}<\frac{1}{b}$.

(5) Si $b<c<a$ la desigualdad es $\frac{1}{a}+\frac{1}{c}+\frac{1}{c} \leq \frac{1}{b}+\frac{1}{b}+\frac{1}{a}$ que es cierta, por ser $\frac{1}{c}<\frac{1}{b}$.

(6) Si $c<b<a$ la desigualdad es $\frac{1}{a}+\frac{1}{b}+\frac{1}{c} \leq \frac{1}{b}+\frac{1}{c}+\frac{1}{a}$ que es cierta.

Por tanto la desigualdad triangular se satisface.

Una vez más, la continuidad de la función $M(a, b, \cdot)$ es evidente.

Finalmente, sean $x \in X, r<\frac{1}{x}$ y $t>0$. Se verifica que $B(x, r, t)=$ ]$\frac{x}{1+r x}, \frac{x}{1-r x}[$. Veámoslo.

$$
\begin{gathered}
B(x, r, t)=\{y \in X: M(x, y, t)>1-r\}= \\
=\left\{y \in X: 1-\left(\frac{1}{x \vee y}-\frac{1}{x \wedge y}\right)>1-r\right\}= \\
=\left\{y \in X:\left(\frac{1}{x \vee y}-\frac{1}{x \wedge y}\right)>r\right\}=
\end{gathered}
$$




$$
\begin{gathered}
=\left\{y \in X: \frac{1}{y}-\frac{1}{x}<r\right\} \cup\left\{y \in X: \frac{1}{x}-\frac{1}{y}<r\right\}= \\
=\left\{y \in X: \frac{1}{y}<r+\frac{1}{x}=\frac{1+r x}{x}\right\} \cup\left\{y \in X: \frac{1}{y}<\frac{1}{x}-r=\frac{1-r x}{x}\right\}= \\
\left.=\left\{y \in X: y>\frac{x}{1+r x}\right\} \cup\left\{y \in X: y<\frac{x}{1-r x}\right\}=\right] \frac{x}{1+r x}, \frac{x}{1-r x}[
\end{gathered}
$$

Por lo tanto $B(x, r, t)$ es un intervalo abierto de $\mathbb{R}$ cuyo diámetro converge a cero siempre que $r$ tiende a infinito y, en consecuencia, $\tau_{M}$ es la topología usual de $\mathbb{R}$ relativa a $X$.

Por otra parte, si en este ejemplo tomamos $a=2, b=3$ y $c=10$, entonces $M(a, b, t) \cdot M(b, c, s)>M(a, c, t+s) \mathrm{y}$, por tanto, $\left(M, T_{2}\right)$ no es una métrica fuzzy.

Ejemplo 1.5.11. Sea $X$ el intervalo $] 2,+\infty[$ de la recta real y sea la aplicación $\left.\left.M: X^{2} \times\right] 0,+\infty[\rightarrow] 0,1\right]$ dada por

$$
M(a, b, t)=\left\{\begin{array}{ccc}
1 & \text { si } & a=b \\
\frac{1}{a}+\frac{1}{b} & \text { si } & a \neq b
\end{array}\right.
$$

Entonces $\left(X, M, T_{3}\right)$ es un espacio métrico fuzzy, mientras que $\left(X, M, T_{2}\right)$ no lo es. Además, la topología $\tau_{M}$ es la topología discreta en $X$.

Demostración. Las propiedades (GV1),(GV2),(GV3) y (GV5) son evidentes por la definición de $M$.

Si $a=b, a=c$ ó $b=c$ la desigualdad triangular resulta obvia.

Si $a \neq b \neq c \neq a$ se tiene

$$
T_{3}(M(a, b, t), M(b, c, s))=\frac{1}{a}+\frac{1}{b}+\frac{1}{b}+\frac{1}{c}-1 \leq \frac{1}{a}+\frac{1}{c}=M(a, c, t+s),
$$


puesto que $b>2$.

Sea $x \in X$. Si tomamos $r<\frac{1}{2}-\frac{1}{x}$, entonces resulta que $B(x, r, t)=\{x\}$ y, en consecuencia, $\tau_{M}$ es la topología discreta en $X$.

Por último, si en este ejemplo tomamos $a=1000, b=3$ y $c=10000$, entonces $M(a, b, t) \cdot M(b, c, s)>M(a, c, t+s)$ y, por tanto, $\left(X, M, T_{2}\right)$ no es un espacio métrico fuzzy.

El ejemplo que presentaremos a continuación es una generalización del obtenido por Gregori y Romaguera en [25].

Ejemplo 1.5.12. Sea $\{A, B\}$ una partición no trivial del intervalo real $X=$ ] $2,+\infty\left[\right.$. Consideremos la aplicación $\left.\left.M: X^{2} \times\right] 0,+\infty[\rightarrow] 0,1\right]$ de la siguiente forma

$$
M(x, y, t)=\left\{\begin{array}{cc}
1-\left(\frac{1}{x \wedge y}-\frac{1}{x \vee y}\right) & \text { si } x, y \in A \text { ó } x, y \in B \\
\frac{1}{x}+\frac{1}{y} & \text { en los demás casos }
\end{array}\right.
$$

Entonces se tiene que $\left(X, M, T_{3}\right)$ es un espacio métrico fuzzy, mientras que $\left(X, M, T_{2}\right)$ no lo es.

Demostración. Con una adecuada modificación de los argumentos aportados por los autores en [25], se prueba que $\left(X, M, T_{3}\right)$ es un espacio métrico fuzzy.

Por otra parte, por el ejemplo 1.5.10, es evidente que $\left(X, M, T_{2}\right)$ no es un espacio métrico fuzzy.

Nota 1.5.13. De los dos ejemplos anteriores se deduce que una base de 
abiertos para el sistema de entornos de un punto $x \in X$ resulta

$$
\begin{aligned}
& \frac{x}{1+r x}, \frac{x}{1-r x}[\bigcap \text { s si } x \in A, y] \frac{x}{1+r x}, \frac{x}{1-r x}[\bigcap B \text { si } x \in B, \\
\operatorname{con} 0 & <r<\frac{1}{2}-\frac{1}{x}
\end{aligned}
$$




\section{Capítulo 2}

\section{Métricas fuzzy no arquimedianas y estándar. Completación de espacios métricos fuzzy}

\subsection{Introducción}

En este capítulo se da una definición de métrica fuzzy no arquimediana, que nos permite caracterizar los espacios metrizables que son fuertemente 0dimensionales, y en la última sección se aborda la completación de espacios métricos fuzzy, y en particular la de los espacios métricos fuzzy estándar. En la segunda sección estudiamos la convergencia de sucesiones de números reales formadas a partir de sucesiones de Cauchy en un espacio métrico fuzzy estándar. La proposición 2.2.1 demuestra que si $\left\{x_{n}\right\}_{n=1}^{\infty}$ e $\left\{y_{n}\right\}_{n=1}^{\infty}$ son dos 
sucesiones de Cauchy en un espacio métrico fuzzy estándar $\left(X, M_{d}, *\right)$, entonces se tiene que la sucesión $\left\{M_{d}\left(x_{n}, y_{n}, t\right)\right\}_{n=1}^{\infty}$ converge a algún número distinto de cero. En cambio, como prueba el contraejemplo 2.2.2, que nos será esencial para la última sección, este resultado no se puede generalizar a un espacio métrico fuzzy cualquiera. En la tercera sección se adapta el concepto de métrica no arquimediana para métricas fuzzy (definición 2.3.1) y se demuestra (proposición 2.3.2) que si $(M, *)$ es una métrica fuzzy entonces $\left(M, T_{1}\right)$ también lo es. La adaptación de la noción de métrica fuzzy no arquimediana resulta satisfactoria puesto que, como se prueba en la proposición 2.3.3, toda métrica fuzzy estándar es no arquimediana si y sólo si lo es la métrica de la que se deduce. Finalmente, retomamos la noción de espacio topológico fuertemente 0-dimensional y en el teorema 2.3.4 se caracterizan los espacios fuertemente 0 -dimensionales que admiten una métrica fuzzy no arquimediana en términos de métrica fuzzy. En la última sección se trata el tema de la completación de un espacio métrico fuzzy $(X, M, *)$ a través de la teoría de isometrías, semejante al caso clásico. Aquí aparece una gran diferencia con la teoría de los espacios métricos, pues como han demostrado Gregori y Romaguera [25], en general un espacio métrico fuzzy no admite completación métrica fuzzy. Nosotros, a imitación de la teoría clásica, decimos que una aplicación entre espacios métricos fuzzy es una isometría (definición 2.4.1) si "preserva" las distancias fuzzy. El espacio métrico fuzzy completo $Y$ se dice que es una completación de $X$ si $X$ puede ser inmerso isométricamente en un subespacio denso de $Y$ (definición 2.4.4). El lema 2.4.5 pone de manifiesto que si $\left\{x_{n}\right\}_{n=1}^{\infty}$ e $\left\{y_{n}\right\}_{n=1}^{\infty}$ son sucesiones de Cauchy en un espacio métrico fuzzy $(X, M, *)$ que admite completación, entonces la sucesión $\left\{M\left(x_{n}, y_{n}, t\right)\right\}_{n=1}^{\infty}$ no puede converger a cero, de lo que se desprende que el espacio métrico fuzzy del ejemplo 1.5.12 no admite completación. No obstante, podemos afirmar (teorema 2.4.7) que si $(X, M, *)$ admite completación, entonces ésta es única (salvo isometría) si $M(x, y, t)$ es una función continua 
de $(x, y)$, de lo que se deduce, conjuntamente con el teorema 2.4.8, que todo espacio métrico fuzzy estándar $\left(X, M_{d}, T_{2}\right)$ admite como completación única $\left(\tilde{X}, M_{\tilde{d}}, T_{2}\right)$ donde $\tilde{d}$ es la completación métrica de la métrica $d$.

\subsection{Una propiedad relativa a la métrica fuzzy estándar}

En esta sección $(X, d)$ será un espacio métrico, y $M_{d}$ la métrica fuzzy estándar deducida de $d$.

Podríamos decir, "grosso modo", que las propiedades de los espacios métricos pueden ser generalizadas a los espacios métricos fuzzy estándar, pero una cuestión obvia es conocer cuáles de estas propiedades pueden ser generalizadas a un espacio métrico fuzzy cualquiera. En esta sección abordaremos una cuestión de este tipo, que nos será de utilidad en la última sección de este capítulo.

Por otra parte, observemos que no hay diferencia significativa entre la métrica fuzzy estándar $M_{d}$ y la métrica fuzzy $\frac{k t^{n}}{k t^{n}+m d(x, y)}$ del ejemplo 1.5.5, salvo que $M_{d}$ es la expresión más simple que depende de $t$.

Es sabido que si $\left\{x_{n}\right\}_{n=1}^{\infty}$ e $\left\{y_{n}\right\}_{n=1}^{\infty}$ son sucesiones de Cauchy en un espacio métrico $(X, d)$, entonces $\left\{d\left(x_{n}, y_{n}\right)\right\}_{n=1}^{\infty}$ es una sucesión que converge en $\mathbb{R}$ con la topología usual. En el caso de un espacio métrico fuzzy estándar se tiene el siguiente resultado.

Proposición 2.2.1. Sean $\left\{x_{n}\right\}_{n=1}^{\infty} e\left\{y_{n}\right\}_{n=1}^{\infty}$ dos sucesiones de Cauchy en un espacio métrico fuzzy estándar $\left(X, M_{d}, T_{2}\right)$ y sea $t>0$. Entonces la suce- 
sión de números reales $\left\{M_{d}\left(x_{n}, y_{n}, t\right)\right\}_{n=1}^{\infty}$ converge a algún número real en el intervalo $] 0,1]$.

Demostración. Si $\left\{x_{n}\right\}_{n=1}^{\infty}$ e $\left\{y_{n}\right\}_{n=1}^{\infty}$ son dos sucesiones de Cauchy en $\left(X, M_{d}, T_{2}\right)$, por la proposición 1.3.11, ambas son de Cauchy en $(X, d)$ y ello implica que $\left\{d\left(x_{n}, y_{n}\right)\right\}_{n=1}^{\infty}$ es una sucesión de Cauchy en $\mathbb{R}$.

Sean $\varepsilon>0$ y $t>0$. Entonces existe $n_{0} \in \mathbb{N}$ tal que

$$
\left|d\left(x_{n}, y_{n}\right)-d\left(x_{m}, y_{m}\right)\right|<\frac{\varepsilon}{t}, \text { para cualesquiera } n, m \geq n_{0}
$$

En consecuencia

$$
\left|\frac{1}{M_{d}\left(x_{n}, y_{n}, t\right)}-\frac{1}{M_{d}\left(x_{m}, y_{m}, t\right)}\right|=\frac{1}{t}\left|d\left(x_{n}, y_{n}\right)-d\left(x_{m}, y_{m}\right)\right|<\varepsilon
$$

para todo $n, m \geq n_{0}$. Ello prueba que la sucesión $\left\{\frac{1}{M_{d}\left(x_{n}, y_{n}, t\right)}\right\}_{n=1}^{\infty}$ es de Cauchy en $\mathbb{R} \mathrm{y}$, por lo tanto es convergente a $k \in \mathbb{R}$. De esto se deduce que la sucesión $\left\{M_{d}\left(x_{n}, y_{n}, t\right)\right\}_{n=1}^{\infty}$ converge a $\left.\left.\frac{1}{k} \in\right] 0,1\right]$, por ser $k \neq \infty$. Además, $M_{d}\left(x_{n}, y_{n}, t\right) \leq 1$ para todo $n \in \mathbb{N}$.

El siguiente contraejemplo demuestra que la anterior proposición no es cierta para cualquier espacio métrico fuzzy.

Contraejemplo 2.2.2. Sea $\{A, B\}$ un partición del intervalo de la recta real $X=] 2,+\infty\left[\right.$ tal que la sucesión $a_{n}=\{2 n-1\}_{n=2}^{\infty} \subset A$ y la sucesión $b_{n}=\{2 n\}_{n=2}^{\infty} \subset B$. Consideremos el espacio métrico fuzzy $\left(X, M, T_{3}\right)$ del ejemplo 1.5.12. Entonces ambas sucesiones son de Cauchy en $\left(X, M, T_{3}\right)$ pero la sucesión $\left\{M\left(a_{n}, b_{n}, t\right)\right\}_{n=1}^{\infty}$ converge a cero.

Demostración. Sea $\varepsilon>0$. Supongamos que $n>m$, entonces:

$$
M\left(a_{n}, a_{m}, t\right)=1-\left(\frac{1}{a_{n} \wedge a_{m}}-\frac{1}{a_{n} \vee a_{m}}\right)=1-\left(\frac{1}{a_{m}}-\frac{1}{a_{n}}\right)=
$$




$$
=1-\left(\frac{1}{2 m-1}-\frac{1}{2 n-1}\right)>1-\varepsilon
$$

siempre que $m>\frac{1+\varepsilon}{2 \varepsilon}$

Análogamente, se prueba que $\left\{b_{n}\right\}_{n=2}^{\infty}$ es de Cauchy en $\left(X, M, T_{3}\right)$.

Por otra parte, es evidente que la sucesión de números reales

$$
\left\{M\left(a_{n}, b_{n}, t\right)\right\}_{n=2}^{\infty}=\left\{\frac{1}{a_{n}}+\frac{1}{b_{n}}\right\}_{n=2}^{\infty}=\left\{\frac{1}{2 n-1}+\frac{1}{2 n}\right\}_{n=2}^{\infty}
$$

converge a cero.

Corolario 2.2.3. Sea $\left\{y_{n}\right\}_{n=1}^{\infty}$ una sucesión de Cauchy en el espacio métrico fuzzy $\left(X, M_{d}, T_{2}\right)$ y sea $a \in X$. Entonces la sucesión de números reales $\left\{M_{d}\left(a, y_{n}, t\right)\right\}_{n=1}^{\infty}$ converge a algún número real en el intervalo $\left.] 0,1\right]$.

Demostración. La sucesión $\left\{x_{n}\right\}_{n=1}^{\infty}=a$ para todo $n \in \mathbb{N}$ es de Cauchy en $\left(X, M_{d}, T_{2}\right)$. Por la anterior proposición, se demuestra que la sucesión de números reales $\left\{M_{d}\left(a, y_{n}, t\right)\right\}_{n=1}^{\infty}$ converge en $\left.] 0,1\right]$.

Anteriormente se ha probado que la proposición 2.2.1 no es generalizable a cualquier tipo de espacio métrico fuzzy. El corolario 2.2.3 plantea la misma pregunta, aunque en este caso sigue siendo una cuestión pendiente.

Cuestión abierta 2.1. Sea $\left\{x_{n}\right\}_{n=1}^{\infty}$ una sucesión de Cauchy en un espacio métrico fuzzy $(X, M, *)$ y sean $a \in X, t>0$. Entonces, ¿Es convergente la sucesión de números reales $\left\{M\left(a, x_{n}, t\right)\right\}_{n=1}^{\infty}$ a algún número real en el intervalo $] 0,1]$ ? 


\subsection{Métricas fuzzy no arquimedianas}

En esta sección vamos a generalizar el concepto de métrica no arquimediana a la teoría de las métricas fuzzy. Veremos la relación existente entre una métrica no arquimediana y su correspondiente métrica fuzzy estándar asociada. Por último, en el teorema 2.3.4, caracterizaremos los espacios fuertemente 0-dimensionales que admiten una métrica fuzzy no arquimediana en términos de métrica fuzzy.

Recordemos que una métrica $d$ en un conjunto $X$ se dice que es no arquimediana si:

$$
d(x, z) \leq \operatorname{máx}\{d(x, y), d(y, z)\}, \text { para cualesquiera } x, y, z \in X
$$

Damos ahora la siguiente definición:

Definición 2.3.1. Sea $(M, *)$ una métrica fuzzy en un conjunto X. Diremos que $(M, *)$ es no arquimediana si:

$M(x, z, t) \geq \operatorname{mín}\{M\{x, y, t), M(y, z, t)\}$, para cualesquiera $x, y, z \in X, t>0$

Proposición 2.3.2. Sea $(M, *)$ una métrica fuzzy no arquimediana en un conjunto $X$. Entonces el par $\left(M, T_{1}\right)$ es una métrica fuzzy en $X$.

Demostración. Por ser $(M, *)$ una métrica fuzzy, $\left(M, T_{1}\right)$ cumple las condiciones (GV1), (GV2), (GV3) y (GV5) de la definición 1.3.1, que son independientes de la $t$-norma continua asociada. Veamos que $\left(M, T_{1}\right)$ cumple la desigualdad triangular.

$$
\begin{aligned}
& M(x, z, t+s) \geq \min \{M(x, y, t+s), M(y, x, t+s)\} \geq \\
& \geq \min \{M(x, y, t), M(y, z, s)\}
\end{aligned}
$$

$\mathrm{y}$, en consecuencia, $\left(M, T_{1}\right)$ es una métrica fuzzy. 
Proposición 2.3.3. Sea d una métrica en un conjunto $X$ y sea $M_{d}$ la correspondiente métrica fuzzy estándar asociada a $d$. Entonces, $d$ es no arquimediana si y sólo si $M_{d}$ es no arquimediana.

Demostración. Supongamos, en primer lugar, que $d$ es no arquimediana. Entonces

$$
\begin{aligned}
M_{d}(x, z, t) & =\frac{t}{t+d(x, z)} \geq \frac{t}{t+\operatorname{máx}\{d(x, y), d(y, z)\}}= \\
& =\min \left\{M_{d}(x, y, t), M_{d}(y, z, t)\right\}
\end{aligned}
$$

y, por tanto, $M_{d}$ es no arquimediana.

Recíprocamente, si $M_{d}$ es no arquimediana, se tiene:

$$
\begin{gathered}
d(x, z)=t\left(\frac{1}{M_{d}(x, z, t)}-1\right) \leq t\left(\frac{1}{\operatorname{mín}\left\{M_{d}(x, y, t), M_{d}(y, z, t)\right\}}-1\right)= \\
=\operatorname{máx}\{d(x, y), d(y, z)\}
\end{gathered}
$$

$y$, en consecuencia, $d$ es no arquimediana.

Recordemos que un espacio completamente regular se dice que es "fuertemente 0-dimensional" si cada 0-conjunto es intersección numerable de conjuntos que son abiertos y cerrados.

Además, sea $(X, \tau)$ un espacio topológico $T_{0}$. Entonces $(X, \tau)$ es "fuertemente 0-dimensional" y metrizable si y sólo si existe una uniformidad $\mathcal{U}$ compatible con $\tau$ que posee una base numerable y transitiva (teorema 6.8 de [17]). Se tiene, entonces, el siguiente teorema.

Teorema 2.3.4. Sea $(X, \tau)$ un espacio topológico. Entonces el espacio $(X, \tau)$ es "fuertemente 0-dimensional" y metrizable si y sólo si la topología $\tau$ se deduce de una métrica fuzzy no arquimediana. 
Demostración. Supongamos que $M$ es una métrica fuzzy no arquimediana compatible con la topología $\tau$.

Gregori y Romaguera probaron en $[24]$ que si $(X, M, *)$ es un espacio métrico fuzzy, entonces la familia $\left\{U_{n}: n \in \mathbb{N}\right\}$ donde

$$
U_{n}=\left\{(x, y) \in X \times X: M\left(x, y, \frac{1}{n}\right)>1-\frac{1}{n}\right\}
$$

es base de una uniformidad $\mathcal{U}$ en $X$ compatible con $\tau_{M}$.

Veamos que, en nuestra situación, la familia $\left\{U_{n}: n \in \mathbb{N}\right\}$ es transitiva.

En efecto, si $(x, y),(y, z) \in U_{n}$, entonces

$$
M\left(x, z, \frac{1}{n}\right) \geq \min \left\{M\left(x, y, \frac{1}{n}\right\}>1-\frac{1}{n}\right.
$$

y, por lo tanto, $(x, z) \in U_{n}$, o, lo que es lo mismo, $U_{n} \circ U_{n}=U_{n}$. En consecuencia, por el mencionado teorema de [17], $(X, \tau)$ es "fuertemente 0-dimensional" y metrizable.

Recíprocamente, supongamos que $(X, \tau)$ es "fuertemente 0-dimensional" y metrizable. Entonces, por el teorema 6.8 de [17], la topología $\tau$ se deduce de una métrica no arquimediana y, por la proposición 2.3.3, $\tau$ se deduce de una métrica fuzzy no arquimediana.

\subsection{Completación de los espacios métricos fuzzy estándar}

En esta sección abordamos el estudio de la completación de los espacios métricos fuzzy. Hemos podido comprobar que, con definiciones adecuadas, 
hasta ahora han podido obtenerse en los espacios métricos fuzzy resultados análogos a los espacios métricos clásicos. Sin embargo, en el caso de la completación, veremos que la situación es distinta y que dicha completación no es posible en general pero que ello sí es posible para espacios métricos fuzzy estándar. Las nociones introducidas en este trabajo generalizan las dadas en [53].

Definición 2.4.1. Sean $(X, M, *)$ y $(Y, N, \star)$ dos espacios métricos fuzzy (en el sentido de George y Veeramani). Diremos que una aplicación $f: X \rightarrow Y$ es una isometría fuzzy si se satisface

$$
N(f(x), f(y), t)=M(x, y, t)
$$

para cualesquiera $x, y \in X, t>0$.

Nota 2.4.2. Si $f: X \rightarrow Y$ es una isometría entonces es $f$ inyectiva. En efecto, si $f(x)=f(y)$ entonces $1=N(f(x), f(y), t)=M(x, y, t) y$, en consecuencia, $x=y$.

Nota 2.4.3. Si $f: X \rightarrow Y$ es una isometría $y\left\{x_{n}\right\}_{n=1}^{\infty}$ es una sucesión de Cauchy en $(X, M, *)$ (en el sentido de George y Veeramani), entonces $\left\{f\left(x_{n}\right)\right\}_{n=1}^{\infty}$ es una sucesión de Cauchy en $(Y, N, \star)$.

En efecto, supongamos que, para cada $t>0, \lim _{n, m \rightarrow \infty} M\left(x_{n}, x_{m}, t\right)=1$. Entonces se tiene que

$$
\lim _{n, m \rightarrow \infty} N\left(f\left(x_{n}\right), f\left(x_{m}\right), t\right)=\lim _{n, m \rightarrow \infty} M\left(x_{n}, x_{m}, t\right)=1 \text {, para todo } t>0 .
$$

Definición 2.4.4. Sea $(X, M, *)$ un espacio métrico fuzzy. Llamamos completación de $X$ a toda cuaterna $(Y, N, \star, f)$ donde $(Y, N, \star)$ es un espacio métrico fuzzy completo y $f$ es una isometría fuzzy de $X$ en un subespacio denso de $Y$. 
Recientemente, los profesores V. Gregori y S. Romaguera [25] han probado que no todo espacio métrico fuzzy admite una completación. Nosotros, basándonos en el siguiente lema, daremos otra prueba (más corta) de que el espacio métrico fuzzy encontrado por dichos profesores no admite completación.

Lema 2.4.5. Sea $(X, M, *)$ un espacio métrico fuzzy que admite completación. Entonces, si $\left\{a_{n}\right\}_{n=1}^{\infty} y\left\{b_{n}\right\}_{n=1}^{\infty}$ son sucesiones de Cauchy en $X, y$ $t>0$, la sucesión de números reales $\left\{M\left(a_{n}, b_{n}, t\right)\right\}_{n=1}^{\infty}$ no puede converger a cero.

Demostración. Supongamos que la cuaterna $(Y, N, \star, f)$ es una completación de $(X, M, *)$. Sean $\left\{a_{n}\right\}_{n=1}^{\infty}$ y $\left\{b_{n}\right\}_{n=1}^{\infty}$ sucesiones de Cauchy en $X$. Entonces, por la proposicion anterior, $\left\{f\left(a_{n}\right)\right\}_{n=1}^{\infty} \mathrm{y}\left\{f\left(b_{n}\right)\right\}_{n=1}^{\infty}$ son sucesiones de Cauchy en $Y$, y, por lo tanto, convergen a $a$ y $b$ respectivamente. Sea $t>0$ y elijamos $\varepsilon$ de manera que $0<\varepsilon<N(a, b, t)$. Sabemos, por continuidad de $M$ respecto de $t$, que existe $\delta>0$ de manera que $N(a, b, t-\delta)>N(a, b, t)-\varepsilon$. Se tiene entonces que

$$
\begin{gathered}
M\left(a_{n}, b_{n}, t\right)=N\left(f\left(a_{n}\right), f\left(b_{n}\right), t\right) \geq \\
\geq N\left(f\left(a_{n}\right), a, \frac{\delta}{2}\right) \star N(a, b, t-\delta) \star N\left(b, f\left(b_{n}\right), \frac{\delta}{2}\right)
\end{gathered}
$$

Cuando $n$ tiende a infinito, es claro que el último miembro de la desigualdad vale $N(a, b, t-\delta)$. Así pues, si existe $\lim _{n \rightarrow \infty} M\left(a_{n}, b_{n}, t\right)$ tiene que ser $\lim _{n \rightarrow \infty} M\left(a_{n}, b_{n}, t\right) \geq N(a, b, t)-\varepsilon>0, \mathrm{y}$, en consecuencia, ha de ser distinto de cero.

Contraejemplo 2.4.6. Consideremos el espacio métrico fuzzy $\left(X, M, T_{3}\right)$ dado en el ejemplo 1.5.12. Como hemos visto en el contraejemplo 2.2.2, se tiene que las sucesiones $\{2 n-1\}$ y $\{2 n\}$ son sucesiones de Cauchy, y además se tiene que

$$
\lim _{n \rightarrow \infty} M\left(a_{n}, b_{n}, t\right)=\left(\frac{1}{2 n-1}+\frac{1}{2 n}\right)=0
$$


por lo que, atendiendo al lema anterior, este espacio métrico fuzzy no admite completación.

Ahora demostraremos en los dos siguientes teoremas que todo espacio métrico fuzzy estándar admite completación única salvo isometría fuzzy.

Teorema 2.4.7. Sea $(X, M, T)$ un espacio métrico fuzzy donde $M(x, y, t)$ es continua respecto de $x$ e $y$. Sean $(Y, N, *, f)$ y $(Z, P, \star, g)$ dos completaciones de $(X, M, T)$. Entonces existe una isometría $h$ de $Y$ en $Z$ tal que $h \circ f=g$. (Si se identifica a $X$ con su imagen isométrica puede decirse que la completación es única salvo isometría fuzzy que deja invariantes los puntos de X).

Demostración. Supongamos que $N$ y $P$ son las métricas fuzzy en $Y$ y $Z$ respectivamente. Definimos $u: f(X) \rightarrow g(X)$ como $u=g \circ f^{-1}$. Veamos que $u$ es una isometría fuzzy. Sean $y_{1}, y_{2} \in f(X)$ y supongamos que $x_{i}=f^{-1}\left(y_{i}\right)$. Entonces

$$
\begin{gathered}
P\left(u\left(y_{1}\right), u\left(y_{2}\right), t\right)=P\left(\left(g \circ f^{-1}\right)\left(y_{1}\right),\left(g \circ f^{-1}\right)\left(y_{2}\right), t\right)= \\
=P\left(g\left(x_{1}\right), g\left(x_{2}\right), t\right)=M\left(x_{1}, x_{2}, t\right)=N\left(f\left(x_{1}\right), f\left(x_{2}\right), t\right)=N\left(y_{1}, y_{2}, t\right)
\end{gathered}
$$

Sea $y \in Y$ y sea $\left\{y_{n}\right\}_{n=1}^{\infty}$ una sucesión en $f(X)$ tal que $\lim _{n \rightarrow \infty} y_{n}=y$. La sucesión $\left\{y_{n}\right\}_{n=1}^{\infty}$ es de Cauchy en $Y$ y, por lo tanto, la sucesión $\left\{u\left(y_{n}\right)\right\}_{n=1}^{\infty}$ es de Cauchy en $Z$ (y, en consecuencia, convergente).

Sea $z=\lim _{n \rightarrow \infty} u\left(y_{n}\right)$. Veamos que $z$ viene determinado por un único $y$ (aunque la sucesión $\left\{y_{n}\right\}_{n=1}^{\infty}$ no sea, necesariamente, única).

Sea $\left\{y_{n}^{\prime}\right\}_{n=1}^{\infty}$ una sucesión en $f(X)$ tal que $\lim _{n \rightarrow \infty} y_{n}^{\prime}=y$. Entonces

$$
N\left(y_{n}, y^{\prime}, t\right) \geq N\left(y_{n}, y, \frac{t}{2}\right) * N\left(y, y_{n}^{\prime}, \frac{t}{2}\right),
$$


que tiende a $1 * 1=1$ cuando $n$ tiende a infinito.

Por lo tanto,

$$
\begin{gathered}
P\left(z, u\left(y_{n}^{\prime}\right), t\right) \geq P\left(\left(z, u\left(y_{n}\right), \frac{t}{2}\right) * P\left(u\left(y_{n}\right), u\left(y_{n}^{\prime}\right), \frac{t}{2}\right)=\right. \\
=P\left(z, u\left(y_{n}\right), \frac{t}{2}\right) * N\left(y_{n}, y_{n}^{\prime}, \frac{t}{2}\right)
\end{gathered}
$$

que tiende a $1 * 1=1$ cuando $n$ tiende a infinito. $\lim _{n \rightarrow \infty} y_{n}$.

Definimos ahora $h: Y \rightarrow Z$ por $h(y)=z=\lim _{n \rightarrow \infty} u\left(y_{n}\right)$, siendo $y=$

Claramente $\left.h\right|_{f(X)}=u$ y, por tanto, $h \circ f=u \circ f=g$. Veamos, por último, que $h$ es una isometría fuzzy. Sean $y, y^{\prime} \in Y$ y sean $\left\{y_{n}\right\}_{n=1}^{\infty}$ e $\left\{y_{n}^{\prime}\right\}_{n=1}^{\infty}$ sucesiones en $f(X)$ tales que $\lim _{n \rightarrow \infty} y_{n}=y$ y $\lim _{n \rightarrow \infty} y_{n}^{\prime}=y^{\prime}$. Entonces

$$
P\left(h(y), h\left(y^{\prime}\right), t\right)=P\left(\lim _{n \rightarrow \infty} u\left(y_{n}\right), \lim _{n \rightarrow \infty} u\left(y_{n}^{\prime}\right), t\right)=(*)
$$

La anterior expresión puede escribirse como sigue puesto que la métrica fuzzy $\mathrm{P}$ es continua respecto de $x, y \in X$ por coincidir con la métrica fuzzy $M$ en el conjunto $X$ y ser $M$ continua respecto de $x, y$.

$$
(*)=\lim _{n \rightarrow \infty} P\left(u\left(y_{n}\right), u\left(y_{n}^{\prime}\right), t\right)=\lim _{n \rightarrow \infty} N\left(y_{n}, y_{n}^{\prime}, t\right)=(\sharp)
$$

Por la misma razón anterior, la métrica fuzzy $N$ es continua en $x, y \in X$ y la última expresión puede escribirse como sigue

$$
(\sharp)=N\left(\lim _{n \rightarrow \infty} y_{n}, \lim _{n \rightarrow \infty} y_{n}^{\prime}, t\right)=N\left(y, y^{\prime}, t\right)
$$

En consecuencia, la aplicación $h$ es una isometría fuzzy, como se quería demostrar.

Teorema 2.4.8. Sea $\left(X, M_{d}, T_{2}\right)$ el espacio métrico fuzzy estándar asociado al espacio métrico $(X, d)$. Sea $(Y, \tilde{d})$ la completación métrica de $(X, d)$. Entonces $\left(Y, M_{\tilde{d}}, T_{2}\right)$ es la completación de $\left(X, M_{d}, T_{2}\right)$, única salvo isometría. 
Demostración. Por ser $(Y, \tilde{d})$ una completación de $(X, d)$, el conjunto $X$ es isométrico a un conjunto denso de $Y$ por medio de una isometría $f$. Veamos que la misma hace que $X$ sea isométrico fuzzy al mismo subconjunto denso de $Y$. En efecto, si $\tilde{d}(f(x), f(y))=d(x, y)$, para cualesquiera $x, y \in X$, entonces

$$
M_{\tilde{d}}(f(x), f(y), t)=\frac{t}{t+\tilde{d}(f(x), f(y))}=\frac{t}{t+d(x, y)}=M_{d}(x, y, t)
$$

Veamos que $\left(Y, M_{\tilde{d}}, T_{2}\right)$ es completo.

Sea $\left\{y_{n}\right\}_{n=1}^{\infty}$ una sucesión de Cauchy en $\left(Y, M_{\tilde{d}}, T_{2}\right)$. Entonces

$$
\begin{gathered}
\lim _{n, m \rightarrow \infty} M_{\tilde{d}}\left(y_{n}, y_{m}, t\right)=1, \text { por lo tanto, } \\
\lim _{n, m \rightarrow \infty} \frac{t}{t+\tilde{d}\left(y_{n}, y_{m}\right)}=1
\end{gathered}
$$

Ello implica que $\lim _{n, m \rightarrow \infty} \tilde{d}\left(y_{n}, y_{m}\right)=0$, es decir, $\left\{y_{n}\right\}_{n=1}^{\infty}$ es de Cauchy en $(Y, \tilde{d})$ y, por ser $(Y, \tilde{d})$ completo, la sucesión $\left\{y_{n}\right\}_{n=1}^{\infty}$ es convergente.

Teniendo en cuenta que $M_{d}(x, y, t)=\frac{t}{t+d(x, y)}$ y que $d(x, y)$ es continua respecto de $x, y$, por el teorema anterior sabemos que dicha completación es única. 


\section{Capítulo 3}

\section{Continuidad uniforme en espacios métricos fuzzy}

\subsection{Introducción}

En este capítulo se define y estudia el concepto de continuidad uniforme (y t-uniforme) entre espacios métricos fuzzy. En la segunda sección se da la definición 3.2.1, que introduce el concepto de aplicación uniformemente continua entre los espacios métricos fuzzy $(X, M, *)$ e $(Y, N, \star)$, y en la proposición 3.3.1 se demuestra que $f: X \rightarrow Y$ es uniformemente continua si y sólo si lo es al considerar en $X$ e $Y$ las uniformidades $\mathcal{U}_{M}$ y $\mathcal{U}_{N}$ deducidas de $M$ y $N$ respectivamente. A continuación, en la definición 3.2.5, se introduce el concepto de métrica fuzzy equinormal, y la proposición 3.2.6, prueba que dicho concepto es acorde con la teoría clásica, pues demuestra que una métrica es equinormal si y sólo si lo es su métrica fuzzy estándar asociada. De la misma forma, en la definición 3.2.7, se adapta la propiedad de Lebesgue 
a la teoría de los espacios métricos fuzzy y la proposición 3.2.8 demuestra que dicha definición es acorde con la teoría clásica puesto que toda métrica cumple la propiedad de Lebesgue si y sólo si la cumple su métrica fuzzy estándar asociada. El resultado fundamental de este capítulo es el teorema 3.2.9 que caracteriza los espacios métricos fuzzy donde las aplicaciones reales continuas son uniformemente continuas en términos de equinormalidad y de la propiedad de Lebesgue. En la tercera sección, la definición 3.3.1 introduce el concepto de aplicación $t$-uniformemente continua entre espacios métricos fuzzy, que resulta un propiedad más fuerte que la de continuidad uniforme. En la proposición 3.3.3 se da una caracterización del concepto de aplicación $t$ uniformemente continua en términos de la métrica del espacio métrico fuzzy. Dicha proposición relaciona este concepto con el de contractividad que veremos en el próximo capítulo, donde se demuestran varios teoremas de punto fijo en espacios métricos fuzzy. La proposición 3.3.4 demuestra que toda aplicación continua de un espacio métrico fuzzy compacto en un espacio métrico fuzzy es $t$-uniformemente continua. Siguiendo el desarrollo de la sección anterior, asociada a la noción de continuidad $t$-uniforme, en la definición 3.3.5 se aporta una noción de $t$-equinormalidad. Dicha definición permite caracterizar, en el teorema 3.3.6, los espacios métricos fuzzy donde toda aplicación real continua es $t$-uniformemente continua en términos de $t$-equinormalidad de la métrica fuzzy. Finalmente se demuestra que el concepto de continuidad t-uniforme resulta más fuerte que el de continuidad uniforme. Para ello, la proposición 3.3.7 aporta un nuevo ejemplo de espacio métrico fuzzy dado por $\left(X, M, T_{2}\right)$, siendo $X=\{1\} \cup\left\{1-\frac{1}{n+1}: n \in \mathbb{N}\right\}$ y $M$ definida por

$$
M(x, y, t)= \begin{cases}1 & \text { si } x=y \\ t x y & \text { si } x \neq y, \quad t<1 \\ x y & \text { si } x \neq y, \quad t \geq 1\end{cases}
$$


que induce la topología discreta (y, en consecuencia, no es un espacio compacto). En la proposición 3.3.8 se prueba que la métrica asociada a dicho ejemplo es equinormal, de lo cual se deduce que toda aplicación continua en $X$ es uniformemente continua. A continuación, en la proposición 3.3.10 se demuestra que la aplicación característica del conjunto $\{1\} \subset X$ es uniformemente continua pero no es $t$-uniformemente continua. También, la proposición 3.3.11 aporta un nuevo ejemplo de aplicación uniformemente continua que no es t-uniformemente continua. Por último, en la proposición 3.3.12 se demuestra que si $(X, M, *)$ es un espacio métrico fuzzy en el que toda aplicación real continua es uniformemente continua, entonces existe una métrica fuzzy $(N, \star)$ (que induce la misma topología que $(M, *)$ en $X$ ) tal que toda aplicación real continua es $t$-uniformemente continua en $(X, N, \star)$.

\subsection{Continuidad uniforme en espacios métri- cos fuzzy}

A continuación damos una definición de continuidad uniforme en términos de métricas fuzzy.

Definición 3.2.1. Diremos que una aplicación $f$ del espacio métrico fuzzy $(X, M, *)$ en el espacio métrico fuzzy $(Y, N, \star)$ es uniformemente continua si para cada $\varepsilon \in] 0,1[y$ cada $t>0$, existen $r \in] 0,1[y s>0$ tales que si $M(x, y, s)>1-r$, entonces $N(f(x), f(y), t)>1-\varepsilon$, para cualesquiera $x, y \in X$.

En particular, diremos que la función $f$ definida en $(X, M, *)$ con valores reales es $\mathbb{R}$-uniformemente continua si para cada $\varepsilon>0$ existen $r \in] 0,1[$ 
y $s>0$, tales que $|f(x)-f(y)|<\varepsilon$ siempre que $M(x, y, s)>1-r$, para cualesquiera $x, y \in X$.

Nota 3.2.2. Por otra parte, es fácil probar que para $r \in] 0,1[y t>0$, el conjunto

$$
U_{r t}=\{(x, y) \in X \times X: M(x, y, t)>1-r\}
$$

es una banda de $\mathcal{U}_{M}$. En efecto, si elegimos $\frac{1}{n} \leq \operatorname{mín}\{r, t\}$, entonces para $(x, y) \in U_{n}$ se tiene que $M(x, y, t) \geq M\left(x, y, \frac{1}{n}\right)>1-\frac{1}{n} \geq 1-r$, por lo que $(x, y) \in U_{r t}$, es decir, $U_{n} \subset U_{r t}$.

En realidad, la anterior definición no es más que una caracterización, en términos de métrica fuzzy, de una función de $X$ en $Y$ que es uniformemente continua respecto de las uniformidades $\mathcal{U}_{M}$ y $\mathcal{U}_{N}$, deducidas de $M$ y $N$ respectivamente, y construidas en la demostración del teorema 1.3.15, como veremos en la próxima proposición.

Proposición 3.2.3. Sean $(X, M, *)$ e $(Y, N, \star)$ dos espacios métricos fuzzy y sea $f: X \rightarrow Y$ una aplicación. Entonces, $f$ es uniformemente continua si y sólo si la aplicación $f:\left(X, \mathcal{U}_{M}\right) \longrightarrow\left(Y, \mathcal{U}_{N}\right)$ es uniformemente continua.

Demostración. Supongamos que $f$ es uniformemente continua respecto de las métricas fuzzy $M$ y $N$. Sea $\left\{V_{n}: n \in \mathbb{N}\right\}$ la base de la uniformidad $\mathcal{U}_{N}$ de $Y$, donde

$$
V_{n}=\left\{\left(y_{1}, y_{2}\right) \in Y \times Y: N\left(y_{1}, y_{2}, \frac{1}{n}\right)>1-\frac{1}{n}\right\}
$$

Tomamos $t=\frac{1}{n}$ y $\varepsilon=\frac{1}{n}$. Entonces, por ser $f$ uniformemente continua, existen $r \in] 0,1\left[, s>0\right.$ tales que si $M\left(x_{1}, x_{2}, s\right)>1-r$, entonces 
$N\left(f\left(x_{1}\right), f\left(x_{2}\right), \frac{1}{n}\right)>1-\frac{1}{n}$.

Sea ahora, $U_{r s}=\left\{\left(x_{1}, x_{2}\right) \in X \times X: M\left(x_{1}, x_{2}, s\right)>1-r\right\} \in \mathcal{U}_{M}$. Entonces, $U_{r s} \subseteq f^{-1}\left(V_{n}\right)=\left\{\left(x_{1}, x_{2}\right) \in X \times X: N\left(f\left(x_{1}\right), f\left(x_{2}\right), \frac{1}{n}\right)>1-\frac{1}{n}\right\}$ y, por lo tanto, $f$ es uniformemente continua respecto de las uniformidades $\mathcal{U}_{M}$ y $\mathcal{U}_{N}$

Recíprocamente, supongamos que $f:\left(X, \mathcal{U}_{M}\right) \longrightarrow\left(Y, \mathcal{U}_{N}\right)$ es uniformemente continua. Sean $\varepsilon \in] 0,1[, t>0$ y sea

$$
V_{\varepsilon t}=\left\{\left(y_{1}, y_{2}\right) \in Y \times Y: M\left(y_{1}, y_{2}, t\right)>1-\varepsilon\right\} \in \mathcal{U}_{N}
$$

Entonces existe $n \in \mathbb{N}$ tal que

$$
\begin{gathered}
f^{-1}\left(V_{\varepsilon t}\right)=\left\{\left(x_{1}, x_{2}\right) \in X \times X: N\left(f\left(x_{1}\right), f\left(x_{2}\right), t\right)>1-\varepsilon\right\} \supset U_{n}= \\
=\left\{(x, y) \in X \times X: M\left(x, y, \frac{1}{n}\right)>1-\frac{1}{n}\right\} .
\end{gathered}
$$

Elijamos $r \in] 0,1\left[\right.$ y $s>0$ de manera que $\frac{1}{n} \geq \operatorname{máx}\{s, r\}$. Si $M(x, y, s)>$ $1-\frac{1}{r}$, entonces $M\left(x, y, \frac{1}{n}\right) \geq M(x, y, s)>1-r>1-\frac{1}{n}$, con lo que $(x, y) \in U_{n}$, y por tanto $N(f(x), f(y), t)>1-\varepsilon$, y en consecuencia $f$ es uniformemente continua respecto de las métricas fuzzy $M$ y $N$.

Es obvio, entonces, que toda función uniformemente continua de $(X, M, *)$ en $(Y, N, \star)$ es continua de $\left(X, \tau_{M}\right)$ en $\left(Y, \tau_{N}\right)$. Además, si $\left(X, \tau_{M}\right)$ es compacto entonces toda función continua de $(X, M, *)$ en $(Y, N, \star)$ es uniformemente continua. (Podemos hacer la prueba sin recurrir a la proposición anterior. En efecto, dados $\varepsilon \in] 0,1[\mathrm{y} t>0$, por la proposición 1.3.4, existe $\delta>0$ tal que $(1-\delta) \star(1-\delta)>1-\varepsilon$. Entonces, para todo $x \in X$, existen 
$\left.r_{x}, r_{x}^{\prime} \in\right] 0,1\left[\right.$ y existe $s_{x}>0$ tales que $f\left(B_{M}\left(x, r_{x}^{\prime}, s_{x}\right)\right) \subseteq B_{N}\left(f(x), \delta, \frac{t}{2}\right)$ $\mathrm{y}$, además, $\left(1-r_{x}\right) *\left(1-r_{x}\right)>1-r_{x}^{\prime}$.

Entonces, existe un un subconjunto finito $A$ de $X$ tal que

$$
X=\bigcup_{x \in A} B_{M}\left(x, r_{x}, \frac{s_{x}}{2}\right)
$$

Sea ahora, $r=\min \left\{r_{x}: x \in A\right\}$ y sea $s=\operatorname{máx}\left\{\frac{s_{x}}{2}: x \in A\right\}$. Es fácil ver que, si $M(x, y, s)>1-r$, entonces $N(f(x), f(y), t)>1-\varepsilon$, y, en consecuencia, $f$ es uniformemente continua.)

Proposición 3.2.4. Sean $(X, d)$ e $(Y, e)$ dos espacios métricos. Entonces la aplicación $f:(X, d) \rightarrow(Y, e)$ es uniformemente continua si y sólo si $f:\left(X, M_{d}, T_{2}\right) \rightarrow\left(Y, M_{e}, T_{2}\right)$ es unformemente continua.

Demostración. Supongamos, en primer lugar, que $f(X, d) \rightarrow(Y, e)$ es uniformemente continua. Sean $\varepsilon \in] 0,1[, t>0$. Entonces existe $\delta \in] 0,1[$ tal que $d(x, y)<\delta$ implica $e(f(x), f(y))<\frac{t \varepsilon}{1-\varepsilon}$.

Tomamos $s=t$ y $\eta=\frac{\delta}{t+\delta}$. Con un sencillo cálculo se prueba que si $M_{d}(x, y, s)>1-\eta$, entonces $d(x, y)<\delta$. Por lo tanto, si $M_{d}(x, y, s)>1-\eta$, se tiene

$$
M_{e}(f(x), f(y), t)=\frac{t}{t+e(f(x), f(y))}>\frac{t}{t+\frac{t \varepsilon}{1-\varepsilon}}=1-\varepsilon
$$

y, en consecuencia, $f:\left(X, M_{d}, T_{2}\right) \rightarrow\left(Y, M_{e}, T_{2}\right)$ es una aplicación uniformemente continua.

Recíprocamente, supongamos que $f:\left(X, M_{d}, T_{2}\right) \rightarrow\left(Y, M_{e}, T_{2}\right)$ es uniformemente continua. Sean $\varepsilon \in] 0,1[\mathrm{y} t>0$. Entonces existen $\eta \in] 0,1[, s>0$ 
tales que $M_{d}(x, y, s)>1-\eta$ implica $M_{e}(f(x), f(y), t)>1-\frac{\varepsilon}{t+\varepsilon}$.

Tomemos $\delta=\frac{s \eta}{1-\eta}$. Entonces, si $d(x, y)<\delta$, se tiene que $M_{d}(x, y, s)>$ $1-\eta$, lo cual implica que $M_{e}(f(x), f(y), t)=\frac{t}{t+e(f(x), f(y))}>1-\frac{\varepsilon}{t+\varepsilon}$, con lo que $e(f(x), f(y))<\varepsilon$, y, por lo tanto, $f:(X, d) \rightarrow(Y, e)$ es uniformemente continua.

Recordemos que una uniformidad $\mathcal{U}$ en $X$ se dice que tiene la propiedad de Lebesgue si para cada cubrimiento abierto $\mathcal{G}$ de $X$ existe $U \in \mathcal{U}$ tal que $\{U(x): x \in X\}$ refina $\mathcal{G}$. Recordemos, por otra parte, que $\mathcal{U}$ se dice que es equinormal si para cada par de subconjuntos no vacíos cerrados disjuntos $A$ y $B$ de $X$ existe $U \in \mathcal{U}$ tal que $U(A) \bigcap B=\emptyset$.

Además, se dice que una métrica $d$ en un conjunto $X$ tiene la propiedad de Lebesgue si la uniformidad $\mathcal{U}_{d}$ deducida de $d$, tiene la propiedad de Lebesgue. De la misma forma, se dice que una métrica $d$ en $X$ es equinormal si la uniformidad $\mathcal{U}_{d}$ deducida de $d$ es equinormal. (ver [17])

En el Teorema 3.2.9 caracterizaremos los espacios métricos fuzzy, en los cuales las funciones reales continuas son uniformemente continuas, en términos de equinormalidad y propiedad de Lebesgue de las métricas fuzzy. Para ello, previamente necesitaremos algunas definiciones.

Definición 3.2.5. Diremos que una métrica fuzzy $(M, *)$ en $X$ es equinormal si para cada par de subconjuntos no vacíos cerrados disjuntos $A$ y $B$ de $\left(X, \tau_{M}\right)$ existe $t>0$ tal que $\sup \{M(a, b, t): a \in A, b \in B\}<1$.

La siguiente proposición pone de manifiesto que la definición dada es acorde con la teoría clásica. 
Proposición 3.2.6. Sea $(X, d)$ un espacio métrico. Entonces la métrica fuzzy estándar $\left(M_{d}, T_{2}\right)$ es equinormal si y sólo si d es equinormal.

Demostración. Supongamos que la métrica fuzzy $\left(M_{d}, T_{2}\right)$ es equinormal. Sean $A$ y $B$ dos cerrados no vacíos disjuntos y sea $\left\{U_{r}^{d}: r>0\right\}$ la base (de $d$-bandas) de la uniformidad $\mathcal{U}_{d}$ deducida de la métrica $d$, donde $U_{r}^{d}=$ $\{(x, y) \in X \times X: d(x, y)<r\}$.

Supongamos que $U_{r}^{d}(A) \cap B \neq \emptyset$, para todo $r>0$. Entonces existe una sucesión $\left\{a_{n}\right\}_{n=1}^{\infty}$ en $A$ y una sucesión $\left\{b_{n}\right\}_{n=1}^{\infty}$ en $B$ tales que $d\left(a_{n}, b_{n}\right)<r$, para todo $r>0$, con lo que ínf $\{d(a, b): a \in A, b \in B\}=0$ y, por lo tanto, para $t>0$,

$$
\sup \left\{M_{d}(a, b, t): a \in A, b \in B\right\}=\sup \left\{\frac{t}{t+d(x, y)}: a \in A, b \in B\right\}=1
$$

que es una contradicción.

Recíprocamente, supongamos que $d$ es equinormal.

Entonces, para cualesquiera $A$ y $B$ cerrados disjuntos, existe $r>0$ tal que $U_{r}^{d}(A) \cap B=\emptyset$, es decir, el conjunto $\{b \in B: d(a, b)<r\}=\emptyset$.

Por tanto, ínf $\{d(a, b): a \in A, b \in B\} \geq r$. En consecuencia, para cualquier $t>0$, se tiene que

$$
\sup \left\{\frac{t}{t+d(a, b)}: a \in A, b \in B\right\} \leq \frac{t}{t+r}<1,
$$

lo cual implica que $M_{d}$ es una métrica fuzzy equinormal.

Definición 3.2.7. Diremos que una métrica fuzzy $(M, *)$ en $X$ tiene la propiedad de Lebesgue si para cada cubrimiento abierto $\mathcal{G}$ de $X$ existen $r \in$ ]0,1[y $s>0$ tales que la familia $\left\{B_{M}(x, r, s): x \in X\right\}$ refina a $\mathcal{G}$. 
La siguiente proposición muestra que la definición dada es acorde con la teoría clásica.

Proposición 3.2.8. Sea $(X, d)$ un espacio métrico. Entonces la métrica fuzzy estándar $\left(M_{d}, T_{2}\right)$ tiene la propiedad de Lebesgue si y sólo si d tiene la propiedad de Lebesgue.

Demostración. Supongamos que la métrica fuzzy $\left(M_{d}, T_{2}\right)$ tiene la propiedad de Lebesgue. Sea $\mathcal{G}$ un cubrimiento de abiertos de $X$. Por hipótesis, existen $r \in] 0,1\left[, s>0\right.$ tales que la familia $\left\{B_{M}(x, r, s): x \in X\right\}$ es un refinamiento de $\mathcal{G}$. Es decir, $\left\{y \in X: \frac{s}{s+d(x, y)}>1-r, x \in X\right\}$ refina a $\mathcal{G}$, o lo que es lo mismo, la familia de $d$-bolas $\left\{B_{d}\left(x, \frac{r s}{1-r}\right): x \in X\right\}$ refina a $\mathcal{G}$ y, por lo tanto, $\left\{U_{\frac{r s}{1-r}}^{d}(x): x \in X\right\}$ es un refinamiento de $\mathcal{G}$. En consecuencia la métrica $d$ tiene la propiedad de Lebesgue.

La prueba del recíproco es similar.

Teorema 3.2.9. Sea $(X, M, *)$ un espacio métrico fuzzy. Entonces los siguientes enunciados son equivalentes:

(1) Para cada espacio métrico fuzzy $(Y, N, \star)$ se verifica que toda función continua de $\left(X, \tau_{M}\right)$ en $\left(Y, \tau_{N}\right)$ es uniformemente continua de $(X, M, *)$ en $(Y, N, \star)$.

(2) Toda función real continua definida en $\left(X, \tau_{M}\right)$ es $\mathbb{R}$-uniformemente continua en $(X, M, *)$. 
(3) Toda función real continua definida en $\left(X, \tau_{M}\right)$ es uniformemente continua en $\left(X, \mathcal{U}_{M}\right)$.

(4) $(M, *)$ es una métrica fuzzy equinormal en $X$.

(5) $\mathcal{U}_{M}$ es una uniformidad equinormal en $X$.

(6) La uniformidad $\mathcal{U}_{M}$ tiene la propiedad de Lebesgue.

(7) La métrica fuzzy $(M, *)$ tiene la propiedad de Lebesgue.

Demostración. (1) $\Longrightarrow(2)$ Sea f una función real continua definida en $\left(X, \tau_{M}\right)$, y sea $\varepsilon>0$. Podemos asumir, sin pérdida de generalidad, que $\varepsilon \in$ ]0, $1[$. Elijamos $t>0$ de manera que $1-\varepsilon>t$.

Al considerar la métrica fuzzy estándar en $\mathbb{R}$, deducida de la métrica usual, por hipótesis, se tiene que existen $r \in] 0,1[$ y $s>0$ tales que si $M(x, y, s)>1-r$ entonces

$$
\frac{t}{t+|f(x)-f(y)|}>1-\varepsilon
$$

Por la elección de $t$, claramente se tiene que $|f(x)-f(y)|<\varepsilon$ siempre que $M(x, y, s)>1-r$, puesto que la anterior desigualdad implica que

$$
|f(x)-f(y)|<\frac{t}{1-\varepsilon}-t=\frac{t \varepsilon}{1-\varepsilon}<\varepsilon
$$

Ello prueba que $f$ es $\mathbb{R}$-uniformemente continua en $(X, M, *)$.

$(2) \Longrightarrow(3)$ Sea $f$ una función real continua en $\left(X, \tau_{M}\right)$ y sea $\varepsilon>0$.

Por hipótesis, sabemos que existen $r \in] 0,1[\mathrm{y} s>0$ tales que

$$
|f(x)-f(y)|<\varepsilon \text { siempre que } M(x, y, s)>1-r \text {. }
$$


Elijamos $n \in \mathbb{N}$ de manera que $\frac{1}{n} \leq \operatorname{mín}\{r, s\}$. Entonces, para cualesquiera $x, y \in X$ tales que $(x, y) \in U_{n}$ se tiene que

$$
M(x, y, s) \geq M\left(x, y, \frac{1}{n}\right)>1-\frac{1}{n} \geq 1-r,
$$

y por tanto $|f(x)-f(y)|<\varepsilon$.

En consecuencia podemos concluir que $f$ es uniformemente continua en $\left(X, \mathcal{U}_{M}\right)$.

$(3) \Longrightarrow(4)$ Sean $A$ y $B$ dos cerrados no vacíos disjuntos de $\left(X, \tau_{M}\right)$. Entonces, como $(X, \tau)$ es un espacio metrizable, es normal y, por tanto, por aplicación del Lema de Uryshon, se tiene que existe una función continua $f: X \longrightarrow[0,1]$ de manera que $f(A) \subset\{0\}$ y $f(B) \subset\{1\}$.

Como, por hipótesis, $f$ es uniformemente continua en $\left(X, \mathcal{U}_{M}\right)$, entonces para $\varepsilon=1$ existe $n \in \mathbb{N}$ tal que $|f(x)-f(y)|<1$ siempre que $M\left(x, y, \frac{1}{n}\right)>$ $1-\frac{1}{n}$

Entonces, $M\left(a, b, \frac{1}{n}\right) \leq 1-\frac{1}{n}$ para todo $a \in A$ y $b \in B$.

Se concluye por tanto que $(M, *)$ es una métrica equinormal en $X$.

$(4) \Longrightarrow(5)$ Supongamos que la uniformidad $\mathcal{U}_{M}$ no es equinormal. Entonces, existen dos cerrados no vacíos disjuntos $A$ y $B$ de $\left(X, \tau_{M}\right)$ de manera que $U(A) \cap B \neq \emptyset$ para toda banda $U \in \mathcal{U}_{M}$.

Fijemos $t>0$. Entonces, con la terminología de la nota 3.2.2 para cada $n \in \mathbb{N}$ se tiene que $U_{\frac{1}{n} t}(A) \cap B \neq \emptyset$, y, por tanto, existe una sucesión $\left\{a_{n}\right\}_{n \in \mathbb{N}}$ en $A$ y una sucesión $\left\{b_{n}\right\}_{n \in \mathbb{N}}$ en B tales que $M\left(a_{n}, b_{n}, t\right)>1-\frac{1}{n}$ para todo 
$n \in \mathbb{N}$.

Ello implica que $\sup \{M(a, b, t): a \in A, b \in B\}=1$, lo cual contradice la hipótesis de que $(M, *)$ sea una métrica equinormal, y, en consecuencia, $\mathcal{U}_{M}$ es una uniformidad equinormal en $X$.

$(5) \Longrightarrow(6)$ (Teorema 2.3 de $[5])$

$(6) \Longrightarrow(7)$ Sea $\mathcal{G}$ un cubrimiento abierto de $X$. Por hipótesis, es obvio que existe $n \in \mathbb{N}$ de manera que $\left\{B_{M}\left(x, \frac{1}{n}, \frac{1}{n}\right): x \in X\right\}$ refina a $\mathcal{G}$.

Por tanto, $(M, *)$ es una métrica fuzzy en $X$ que tiene la propiedad de Lebesgue.

$(7) \Longrightarrow(1)$ Sea $(Y, N, \star)$ un espacio métrico fuzzy y sea $f$ una función continua de $\left(X, \tau_{M}\right)$ en $\left(Y, \tau_{M}\right)$. Fijemos $\left.\varepsilon \in\right] 0,1[\mathrm{y} t>0$, y consideremos $\delta \in] 0,1[$ tal que $(1-\delta) \star(1-\delta)>1-\varepsilon$.

Puesto que $f$ es continua, para cada $x \in X$ existe un entorno abierto $V_{x}$ de $x$ tal que $f\left(V_{x}\right) \subset B_{N}\left(f(x), \delta, \frac{t}{2}\right)$.

Por hipótesis, existen $r=r(t, \varepsilon) \in] 0,1[$ y $s>0$ tales que la familia $\left\{B_{M}(x, r, s): x \in X\right\}$ refina a $\left\{V_{x}: x \in X\right\}$. Ahora bien, si $M(x, y, s)>1-r$, tendremos que $y \in B_{M}(x, r, s)$, y así, $x, y \in V_{z}$ para algún $z \in X$.

Por tanto, $f(x)$ y $f(y)$ están en $B_{N}\left(f(z), \delta, \frac{t}{2}\right)$, y en consecuencia:

$$
\begin{aligned}
N(f(x), f(y), t) & \geq N\left(f(x), f(z), \frac{t}{2}\right) \star N\left(f(z), f(y), \frac{t}{2}\right) \geq \\
& \geq(1-\delta) \star(1-\delta)>1-\varepsilon,
\end{aligned}
$$


y queda, por tanto, demostrado que f es uniformemente continua de $(X, M, *)$ en $(Y, N, \star)$.

Se sabe (ver [5]) que todo espacio topológico metrizable admite una métrica con la propiedad de Lebesgue si y sólo si el conjunto de puntos no aislados es compacto. Entonces, según el teorema precedente, se tiene el siguiente corolario.

Corolario 3.2.10. Un espacio topológico fuzzy-metrizable admite una métrica fuzzy con la propiedad de Lebesgue si y sólo si el conjunto de puntos no aislados es compacto.

Nota 3.2.11. Dado un espacio topológico metrizable $(X, \tau)$, denotamos por $\mathcal{F N}_{M}$ al supremo de todas las uniformidades $\mathcal{U}_{M}$ inducidas por todas las métricas fuzzy compatibles en $X$. Es fácil ver que $\mathcal{F N}_{M}$ es la uniformidad más fina en $X$. En consecuencia, el teorema clásico de que si un espacio topológico $X$ admite una métrica d con la propiedad de Lebesgue, entonces la uniformidad $\mathcal{U}_{d}$ coincide con la uniformidad más fina de $X$, puede reformularse como sigue:

Si un espacio topológico admite una métrica fuzzy $(M, *)$ con la propiedad de Lebesgue, entonces la uniformidad $\mathcal{U}_{M}$ coincide con la uniformidad $\mathcal{F N}_{M}$.

\subsection{Continuidad $t$-uniforme en espacios mé- tricos fuzzy}

En esta sección introducimos el concepto de aplicación $t$-uniformemente continua, que está estrechamente relacionado con el de aplicación contractiva fuzzy que utilizaremos en el próximo capítulo para obtener teoremas de punto 
fijo en espacios métricos fuzzy completos. Esta noción, más fuerte que la de aplicación uniformemente continua, aporta nuevos resultados y propiedades diferentes de los vistos hasta ahora en el presente capítulo. Veremos que toda aplicación continua en un espacio métrico fuzzy compacto es $t$-uniformemente continua y, además, obtendremos una caracterización de aquellos espacios métricos fuzzy para los cuales toda función real continua es $t$-uniformemente continua en términos de una adecuada noción de $t$-equinormalidad.

Definición 3.3.1. Sea $f$ una aplicación de un espacio métrico fuzzy $(X, M, *)$ en un espacio métrico fuzzy $(Y, N, \star)$. Diremos que $f$ es t-uniformemente continua si dado $\varepsilon \in] 0,1[$ existe $r \in] 0,1[$ tal que si $x, y \in X, t>0 y$ $M(x, y, t)>1-r$, entonces $N(f(x), f(y), t)>1-\varepsilon$.

Nota 3.3.2. Claramente, si $f$ es una aplicación t-uniformemente continua de $(X, M, *)$ en $(Y, N, \star)$, entonces $f$ es uniformemente continua para las uniformidades $\mathcal{U}_{M} y \mathcal{U}_{N}$, generadas por $M y N$, en consecuencia, $f$ es continua para las topologías $\tau_{M}$ y $\tau_{N}$, deducidas de $M$ y $N$ respectivamente.

Proposición 3.3.3. Sea $f$ una aplicación de un espacio métrico fuzzy $(X, M, *)$ en un espacio métrico fuzzy $(Y, N, \star)$. Entonces $f$ es t-uniformemente continua si y sólo si dado $\delta>0$, existe $\eta>0$ tal que

$$
\text { si } x, y \in X, t>0 \text { y } \frac{1}{M(x, y, t)}-1<\eta \text {, entonces } \frac{1}{N(f(x), f(y), t)}-1<\delta
$$

Demostración. Sea $\delta>0$. Consideramos $0<\varepsilon=1-\frac{1}{1+\delta}<1$.

Como $f$ es $t$-uniformemente continua, entonces existe $r \in] 0,1[$ tal que si $x, y \in X, t>0$, y $M(x, y, t)>1-r$, entonces $N(f(x), f(y), t)>1-\varepsilon$. 
Sea $\eta=\frac{1}{1-r}-1>0$. Entonces, si $\frac{1}{M(x, y, t)}-1<\eta$ se tiene que $M(x, y, t)>1-r$, que, a su vez implica que $N(f(x), f(y), t)>1-\varepsilon$, y, en consecuencia

$$
\frac{1}{N(f(x), f(y), t)}-1<\frac{1}{1-\varepsilon}-1=\delta
$$

Recíprocamente, sea $\varepsilon \in] 0,1\left[\right.$. Consideramos $0<\delta<\frac{1}{1-\varepsilon}-1$. Entonces existe $\eta>0$ tal que si $x, y \in X, t>0 \mathrm{y}$

$$
\frac{1}{M(x, y, t)}-1<\eta \text {, se cumple que } \frac{1}{N(f(x), f(y), t)}-1<\delta
$$

Sea ahora $0<r=1-\frac{1}{\eta+1}<1$. Entonces,

$$
M(x, y, t)>1-r=\frac{1}{1+\eta}
$$

y, por lo tanto,

En consecuencia,

$$
\frac{1}{M(x, y, t)}-1<\eta
$$

$$
\frac{1}{N(f(x), f(y), t)}-1<\delta
$$

que implica

$$
N(f(x), f(y), t)>1-\varepsilon .
$$

Como se quería demostrar.

Proposición 3.3.4. Sea $(X, M, *)$ un espacio métrico fuzzy compacto. Entonces, toda aplicación continua $f$ de $(X, M, *)$ en un espacio métrico fuzzy $(Y, N, \star)$ es t-uniformemente continua.

Demostración. Supongamos que existe una aplicación continua $f$ del espacio métrico fuzzy compacto $(X, M, *)$ en el espacio métrico fuzzy $(Y, N, \star)$ que no es $t$-uniformemente continua. 
Entonces, existen $\varepsilon \in] 0,1\left[, t>0\right.$ y dos sucesiones $\left\{x_{n}\right\}_{n=1}^{\infty}$ e $\left\{y_{n}\right\}_{n=1}^{\infty}$ en $X$ tales que $M\left(x_{n}, y_{n}, t\right)>1-\frac{1}{n}$ y que verifican $N\left(f\left(x_{n}\right), f\left(y_{n}\right), t\right) \leq 1-\varepsilon$ para todo $n \in \mathbb{N}$.

Por la compacidad de $\left(X, \tau_{M}\right)$, existen dos subsucesiones $\left\{x_{n_{k}}\right\}_{k \in \mathbb{N}}$ e $\left\{y_{n_{k}}\right\}_{k \in \mathbb{N}}$ de $\left\{x_{n}\right\}_{n \in \mathbb{N}}$ e $\left\{y_{n}\right\}_{n \in \mathbb{N}}$ respectivamente, y existen $x, y \in X$ tales que $\left\{x_{n_{k}}\right\}$ converge a $x$ e $\left\{y_{n_{k}}\right\}$ converge a $y$ en $\left(X, \tau_{M}\right)$.

Puesto que $M(x, y, 3 t) \geq M\left(x, x_{n_{k}}, t\right) * M\left(x_{n_{k}}, y_{n_{k}}, t\right) * M\left(y_{n_{k}}, y, t\right)$, se sigue que $M(x, y, 3 t)=1 \mathrm{y}$, en consecuencia, $x=y$. Entonces, por la continuidad de $f$, se tiene que $\left\{f\left(x_{n_{k}}\right)\right\}$ converge a $f(x)$ y $\left\{f\left(y_{n_{k}}\right)\right\}$ converge a $f(y)$ en $\left(Y, \tau_{N}\right)$.

Sea ahora, $\delta>0$ tal que $(1-\delta) *(1-\delta)>1-\varepsilon$. Entonces, existe $k_{0} \in \mathbb{N}$ tal que $N\left(f(x), f\left(x_{n_{k}}\right), \frac{t}{2}\right)>1-\delta$ y $N\left(f(x), f\left(y_{n_{k}}\right), \frac{t}{2}\right)>1-\delta$ para todo $k \geq k_{0}$. Por lo tanto,

$$
\begin{aligned}
N\left(f\left(x_{n_{k}}\right), f\left(y_{n_{k}}\right), t\right) & \geq N\left(f\left(x_{n_{k}}\right), f(x), \frac{t}{2}\right) \star N\left(f(x), f\left(y_{n_{k}}\right), \frac{t}{2}\right) \geq \\
& \geq(1-\delta) \star(1-\delta)>1-\varepsilon
\end{aligned}
$$

para todo $k \geq k_{0}$, lo cual es una contradicción. En consecuencia, la aplicación $f$ es $t$-uniformemente continua.

Definición 3.3.5. Una métrica fuzzy $(M, *)$ en un conjunto $X$ es t-equinormal si para cada par de subconjuntos no vacíos, cerrados y disjuntos $A$ y $B$ de $\left(X, \tau_{M}\right)$ y cada $t>0$, entonces

$$
\sup \{M(a, b, t): a \in A, b \in B\}<1
$$

Teorema 3.3.6. Sea $(X, M, *)$ un espacio métrico fuzzy. Entonces los siguientes enunciados son equivalentes: 
(1) Para cualquier espacio métrico fuzzy $(Y, N, \star)$, toda aplicación continua de $\left(X, \tau_{M}\right)$ en $\left(Y, \tau_{N}\right)$ es t-uniformemente continua de $(X, M, *)$ en $(Y, N, \star)$.

(2) Toda función real continua de $\left(X, \tau_{M}\right)$ es t-uniformemente continua de $(X, M, *)$ en el espacio métrico fuzzy euclídeo $\left(\mathbb{R}, M_{|\cdot|}, \cdot\right)$.

(3) La métrica fuzzy $(M, *)$ es t-equinormal.

Demostración. (1) $\Longrightarrow(2)$ Obvio.

$(2) \Longrightarrow(3)$ Sean $A$ y $B$ dos subconjuntos no vacíos, cerrados y disjuntos de $\left(X, \tau_{M}\right)$ y fijemos $t>0$. Sea $f: X \rightarrow[0,1]$ una aplicación continua tal que $f(A) \subseteq\{0\}$ y $f(B) \subseteq\{1\}$. Tomamos, $\varepsilon=\frac{1}{t+1}$. Por hipótesis, existe $r \in] 0,1[$ tal que

$$
\frac{t}{t+|f(x)-f(y)|}>1-\varepsilon
$$

siempre que $M(x, y, t)>1-r$.

Para cada $a \in A$ y $b \in B$ se tiene que $\frac{t}{t+|f(a)-f(b)|}=\frac{t}{t+1}=1-\varepsilon$ y, por lo tanto, $M(a, b, t) \leq 1-r$ para cualesquiera $a \in A$ y $b \in B$. En consecuencia, $(M, *)$ es una métrica fuzzy $t$-equinormal en $X$.

$(3) \Longrightarrow(1)$ Supongamos que existen un espacio métrico fuzzy $(Y, N, \star)$ y una aplicación continua $f$ de $\left(X, \tau_{M}\right)$ en $\left(Y, \tau_{N}\right)$ que no es $t$-uniformemente continua. Entonces existen $\varepsilon \in] 0,1\left[, t>0\right.$ y dos sucesiones $\left\{a_{n}\right\}_{n=1}^{\infty}$ y $\left\{b_{n}\right\}_{n=1}^{\infty}$ tales que $M\left(a_{n}, b_{n}, t\right)>1-\frac{1}{n}$ y siendo $N\left(f\left(a_{n}\right), f\left(b_{n}\right), t\right) \leq 1-\varepsilon$ para todo $n \in \mathbb{N}$.

Distinguiremos dos casos: 
Caso 1. La sucesión $\left\{a_{n}\right\}_{n=1}^{\infty}$ tiene una subsucesión $\left\{a_{n_{k}}\right\}_{k \in \mathbb{N}}$ con límite $a \in X$.

Si la sucesión $\left\{b_{n_{k}}\right\}_{k \in \mathbb{N}}$ posee un punto de acumulación $b \in X$, entonces, procediendo de la misma forma que en la proposición 3.3.4, se obtiene que $M(a, b, 3 t)=1 \mathrm{y}$, en consecuencia, $a=b$, y por continuidad de la aplicación $f$, se tiene que $N\left(f\left(a_{n_{k}}\right), f\left(b_{n_{k}}\right), t\right)>1-\varepsilon$, lo cual es una contradicción.

En otro caso, podemos suponer, sin pérdida de generalidad, que $\{a\} \cup$ $\left\{a_{n_{k}}: k \in \mathbb{N}\right\}$ y $\left\{b_{n_{k}}: k \in \mathbb{N}\right\}$ son subconjuntos cerrados y disjuntos de $X$. Pero, en este caso, $\sup \left\{M\left(a_{n_{k}}, b_{n_{k}}, t\right): k \in \mathbb{N}\right\}=1$ lo cual lleva a una contradicción.

Caso 2. La sucesión $\left\{a_{n}\right\}_{n=1}^{\infty}$ no tiene una subsucesión convergente.

Entonces podemos suponer, sin pérdida de generalidad, que $\left\{a_{n}: n \in \mathbb{N}\right\}$ y $\overline{\left\{b_{n}: n \in \mathbb{N}\right\}}$ son subconjuntos cerrados disjuntos de $X$. En consecuencia, nuevamente obtenemos un contradicción.

Por lo tanto, $f$ es una aplicación $t$-uniformemente continua de $(X, M, *)$ en $(Y, N, \star)$.

Proposición 3.3.7. Sea $X=\{1\} \cup\left\{1-\frac{1}{n+1}: n \in \mathbb{N}\right\}$ y sea la aplicación $M: X \times X \times] 0,+\infty[\rightarrow] 0,1]$ dada por:

$$
M(x, y, t)=\left\{\begin{array}{ccl}
1 & \text { si } & x=y \\
t x y & \text { si } & x \neq y, \quad t<1 \\
x y & \text { si } & x \neq y, \quad t \geq 1
\end{array}\right.
$$

Entonces la terna $\left(X, M, T_{2}\right)$ es un espacio métrico fuzzy que genera la 
topología discreta.

Demostración. Es obvio que $M(x, y, t)>0$ para cualesquiera $x, y \in X$, $t>0$. Además, por la definición, es evidente que $M(x, y, t)=1$ si y sólo si $x=y$. La simetría de $M$ y la continuidad de la aplicación $M(x, y, \cdot)$ son triviales. Por último, veamos que se cumple la desigualdad triangular. Supongamos que $x \neq y, y \neq z, x \neq z$.

Si $t, s \geq 1$, entonces $M(x, y, t) \cdot M(y, z, s)=x y \cdot y z \leq x z=M(x, z, t+s)$.

Si $t<1, s \geq 1$, entonces $M(x, y, t) \cdot M(y, z, s)=t x y \cdot y z \leq x z=$ $M(x, z, t+s)$.

Si $t, s<1$, entonces se tienen dos casos:

$$
\begin{aligned}
& t+s \geq 1, M(x, y, t) \cdot M(y, z, s)=t x y \cdot s y z \leq x z=M(x, z, t+s) . \\
& t+s<1, M(x, y, t) \cdot M(y, z, s)=t x y \cdot s y z \leq t x z \geq(t+s) x z=
\end{aligned}
$$
$M(x, z, t+s)$.

Los casos $x=y, x=z$, e $y=z$ resultan evidentes.

Veamos que la métrica fuzzy $\left(M, T_{2}\right)$ genera la topología discreta.

Sea $x \in X$. Tomamos $t<1$ arbitrario y $r=1-t x \in] 0,1[$. Con ellos resulta que

$$
\begin{gathered}
B(x, 1-t x, t)=\{x\} \cup\left\{y \in X: y>\frac{1-(1-t x)}{t x}\right\}= \\
=\{x\} \cup\{y \in X: y>1\}=\{x\} .
\end{gathered}
$$


En consecuencia, $\{x\} \in \tau_{M}$, para todo $x \in X$ y por tanto $\tau_{M}$ es la topología discreta.

Proposición 3.3.8. La métrica fuzzy $\left(M, T_{2}\right)$ de la proposición anterior es equinormal.

Demostración. Sean $A$ y $B$ dos conjuntos cerrados disjuntos no vacíos.

Sea $s<1$ y tomamos $t<s$. Entonces, para cualesquiera $a \in A, b \in B$ se tiene que $M(a, b, t)=t a b<t<s$.

Ello implica que $\sup \{M(a, b, t): a \in A, b \in B\} \leq s<1$ y, en consecuencia, $\left(M, T_{2}\right)$ es una métrica fuzzy equinormal.

Corolario 3.3.9. Toda función real en $\left(X, M, T_{2}\right)$ es uniformemente continua.

Demostración. En efecto, cualquier función definida en $X$ es continua por ser $\tau_{M}$ la topología discreta. El resultado es ahora consecuencia inmediata de la equinormalidad de la métrica fuzzy $\left(M, T_{2}\right)$ y el teorema 3.2.9

Proposición 3.3.10. Sea $\left(X, M, T_{2}\right)$ el espacio métrico fuzzy de la proposición 3.3.7 y sea $f$ la función característica del conjunto $\{1\}$, es decir $f(1)=1$ y $f(x)=0$ para todo $x \in X \backslash\{1\}$. Entonces $f$ es uniformemente continua pero no es t-uniformemene continua.

Demostración. Por el corolario anterior, $f$ es uniformemente continua. Por otra parte, sea $\varepsilon=\frac{1}{2}$ y sea $t=1$. Entonces, para cualquier $\left.r \in\right] 0,1[$, existe $n \in \mathbb{N}$ tal que $\frac{1}{n+1}<r y$, por lo tanto, $M\left(1,1-\frac{1}{n+1}, t\right)>1-r$. 
Sin embargo, $M_{|\cdot|}\left(f(1), f\left(1-\frac{1}{n+1}\right), t\right)=M_{|\cdot|}(1,0,1)=\frac{1}{2}=1-\varepsilon$. Por lo tanto, $f$ no es $t$-uniformemente continua de $(X, M, *)$ en $\left(\mathbb{R}, M_{|\cdot|}, \cdot\right)$.

Proposición 3.3.11. Sea $\left(X, M, T_{2}\right)$ el espacio métrico fuzzy de la proposición 3.3.7 y sea la aplicación $f: X \rightarrow X$ dada por:

$$
\begin{aligned}
& f\left(\frac{n}{n+1}\right)=\left\{\begin{array}{lll}
\frac{1}{2} & \text { si } & \text { n es impar } \\
\frac{n}{n+1} & \text { si } & \text { n es par }
\end{array}\right. \\
& f(1)=1
\end{aligned}
$$

Entonces $f$ es uniformemente continua pero no es t-uniformemente continua.

Demostración. Veamos que $f$ es uniformemente continua.

Dados $t<1, \varepsilon \in] 0,1\left[\right.$, tomamos $s=\frac{t}{2}, r=\varepsilon$.

Supongamos que $M(x, y, s)>1-r$, entonces se tiene que sxy $>1-r$. Por ello,

Si $M(f(x), f(y), t)=t \frac{1}{2} y=\frac{t}{2} y=s y \geq s x y>1-r=1-\varepsilon$

Si $M(f(x), f(y), t)=t x \frac{1}{2}=\frac{t}{2} x=s x \geq s x y>1-r=1-\varepsilon$

Si $M(f(x), f(y), t)=M\left(\frac{1}{2}, \frac{1}{2}, t\right)=1>1-\varepsilon$

Si $M(f(x), f(y), t)=M(x, y, t)=t x y \geq s x y>1-r=1-\varepsilon$. 
En consecuencia, la aplicación $f$ es uniformemente continua.

Veamos que no es $t$-uniformemente continua.

Sean $t=1, \varepsilon=\frac{1}{2}$.

Supongamos que existe $r \in] 0,1[$ tal que si $M(x, y, 1)>1-r$, entonces $M(f(x), f(y), 1)>1-\varepsilon=\frac{1}{2}$.

Sea $n>\frac{2(1-r)}{r}$ par, y sean $x=\frac{n}{n+1}$, e $y=\frac{n+1}{n+2}$. Entonces se tiene que $M(x, y, 1)>1-r$. Ahora bien,

$$
M(f(x), f(y), 1)=\frac{n}{(n+1)} \frac{1}{2}=\frac{n}{2(n+1)} \leq \frac{1}{2}
$$

que es una contradicción. Por tanto $f$ no es $t$-uniformemente continua.

Proposición 3.3.12. Sea $(X, M, *)$ un espacio métrico fuzzy en el que toda función real continua es uniformemente continua de $(X, M, *)$ en $\left(\mathbb{R}, M_{|\cdot|}, \cdot\right)$. Entonces existe una métrica fuzzy $(N, \star)$ en $X$ compatible con la topología $\tau_{M}$ para la que toda función real continua en $\left(X, \tau_{M}\right)$ es t-uniformemente continua de $(X, N, \star)$ en el espacio métrico fuzzy euclídeo.

Demostración. Sabemos que la familia $\left\{U_{n}: n \in \mathbb{N}\right\}$ es base numerable de una uniformidad $\mathcal{U}_{M}$ en $X$ compatible con la topología $\tau_{M}$, donde $U_{n}=$ $\left\{(x, y) \in X \times X: M\left(x, y, \frac{1}{n}\right)>1-\frac{1}{n}\right\}$ para todo $n \in \mathbb{N}$. Se sabe [34] que existe una métrica $d$ en $X$ tal que para cada $n \in \mathbb{N}$,

$$
U_{n+1} \subseteq\left\{(x, y) \in X \times X: d(x, y)<2^{-n}\right\} \subseteq U_{n}
$$


En consecuencia, la topología $\tau_{d}$ inducida por la métrica $d$ coincide con la topología $\tau_{M}$. Más aún, toda función real continua en el espacio métrico $(X, d)$ es uniformemente continua y, por lo tanto, $d$ es una métrica equinormal en $X$ (véase, por ejemplo [5]). Si $\left(M_{d}, T_{2}\right)$ es la métrica fuzzy estándar inducida por $d$, entonces $\left(M_{d}, T_{2}\right)$ es compatible con la topología $\tau_{M}$.

Veamos ahora que $\left(M_{d}, T_{2}\right)$ es $t$-equinormal.

Sean $A$ y $B$ dos subconjuntos no vacíos, cerrados y disjuntos de $X$ y sea $t>0$. Por la equinormalidad de la métrica $d$, existe $\delta>0$ tal que $d(a, b) \geq \delta$ para todo $a \in A, b \in B$. Entonces, para cualesquiera $a \in A$ y $b \in B$ se tiene

$$
M_{d}(a, b, t)=\frac{t}{t+d(a, b)} \leq \frac{t}{t+\delta}
$$

$\mathrm{y}$, en consecuencia, $\sup \left\{M_{d}(a, b, t): a \in A, b \in B\right\}<1 \mathrm{y}$, por el teorema 3.3.6, toda función real continua en $\left(X, \tau_{M_{d}}\right)$ es $t$-uniformemente continua de $\left(X, M_{d}, T_{2}\right)$ en el espacio métrico fuzzy euclídeo. 


\section{Capítulo 4}

\section{Teoremas de punto fijo en espacios métricos fuzzy completos}

\subsection{Introducción}

Este capítulo está conformado por cuatro secciones que tratan de aportar nuevos resultados acerca de teoremas de punto fijo para aplicaciones contractivas fuzzy en espacios métricos fuzzy completos.

La segunda sección de este capítulo introduce, en la definición 4.2.1, el concepto de aplicación contractiva fuzzy en espacios métricos fuzzy. Más concretamente, diremos que $f: X \rightarrow X$ es contractiva fuzzy en $(X, M, *)$ si 
existe $k \in] 0,1[$ tal que se satisface

$$
\frac{1}{M(f(x), f(y), t)}-1<k\left(\frac{1}{M(x, y, t)}-1\right)
$$

para cualesquiera $x, y \in X, t>0$. Esta definición es adecuada en el contexto de los espacios métricos fuzzy, en un doble sentido. En primer lugar, la proposición 4.2.2 demuestra que toda aplicación contractiva fuzzy es $t$-uniformemente continua, y además, porque, con esta definición, una aplicación es contractiva en un espacio métrico si y sólo si es contractiva fuzzy en el espacio métrico fuzzy estándar asociado (proposición 4.2.3). Por último, en la definicion 4.2.5, se introduce el concepto de sucesión contractiva fuzzy en un espacio métrico fuzzy que resulta de interés en las demostraciones de los teoremas de punto fijo de las siguientes secciones (objetivo del capítulo). Tal concepto, también es acorde con la teoría clásica, pues una sucesión es contractiva en un espacio métrico si y sólo si lo es en el correspondiente espacio métrico fuzzy estándar asociado tal como lo prueba la proposición 4.2.6.

En la tercera sección se dan teoremas de punto fijo en espacios métricos fuzzy de George y Veeramani para aplicaciones contractivas fuzzy en el sentido introducido en la sección anterior. En el teorema 4.3.1 se prueba la existencia de un único punto fijo para aplicaciones contractivas fuzzy en espacios métricos fuzzy completos donde toda sucesión contractriva fuzzy es una sucesión de Cauchy. Este resultado permite extender el conocido teorema de punto fijo de Banach a aplicaciones contractivas fuzzy en espacios métricos fuzzy completos. A continuación, este teorema se ilustra con el ejemplo 4.3.3, que justifica las condiciones impuestas en su enunciado. El teorema 4.3.4 es una generalización del anterior y prueba la existencia y unicidad de un punto fijo común para una familia numerable de aplicaciones contractivas fuzzy. En el teorema 4.3.6 demostramos la existencia y unicidad de un punto fijo para 
aplicaciones que satisfacen la condición $M(f(x), f(y), k t)>M(x, y, t)$ para cualesquiera $x, y \in X, t>0$ debida a Sehgal y Bahrucha-Reid [47]. Dicha condición (que podemos considerar como otra condición de contractividad de la aplicación $f$ ) resulta ser equivalente a la contractividad fuzzy introducida anteriormente en los espacios métricos fuzzy estándar (como demuestra la proposición 4.3.9). Para poder justificar la existencia de espacios métricos fuzzy que satisfacen las condiciones del teorema, incluimos el ejemplo 4.3.8, que aporta dos espacios métricos fuzzy, uno de los cuales no satisface las condiciones del teorema (de hecho, en este espacio, la aplicación identidad satisface la condición de contractividad impuesta pero, obviamente, no posee un único punto fijo), mientras que el segundo de ellos sí que las satisface.

Por último, la cuarta sección tiene como objetivo extender el teorema de punto fijo de Banach a aplicaciones contractivas fuzzy en espacios métricos fuzzy de Kramosil y Michalek (definición 1.2.10) que son completos en el sentido de Grabiec. La definición 4.4.1 introduce el concepto de sucesión de Cauchy aportado por Grabiec. A continuación se da el teorema 4.4.4 donde se demuestra la existencia de un único punto fijo para aplicaciones contractivas fuzzy en espacios métricos fuzzy de Kramosil y Michalek con la condición de completitud de Grabiec. Finalmente se adapta el teorema de Edelstein [12] aportado por Grabiec en [23] con la condición de contractividad introducida en este trabajo.

\subsection{Contracción en espacios métricos fuzzy}

Empezamos la sección definiendo el concepto de aplicación contractiva fuzzy. 
Definición 4.2.1. Sea $(X, M, *)$ un espacio métrico fuzzy. Diremos que una aplicación $f: X \longrightarrow X$ es contractiva fuzzy si existe $k \in] 0,1[$ tal que:

$$
\frac{1}{M(f(x), f(y), t)}-1<k\left(\frac{1}{M(x, y, t)}-1\right)
$$

para cualesquiera $x, y \in X, t>0$.

La constante $k$ se llama constante de contractividad de la aplicación $f$.

Proposición 4.2.2. Sea $(X, M, *)$ un espacio métrico fuzzy. Si $f: X \longrightarrow X$ es una aplicación contractiva fuzzy entonces $f$ es t-uniformemente continua.

Demostración. Sea $\delta>0$. Supongamos que $k \in] 0,1[$ es la constante de contractividad de $f$, y elijamos $\eta=\frac{\delta}{k}>0$. Supongamos que para $x, y \in$ $X, t>0$ se tiene que $\frac{1}{M(x, y, t)}-1<\eta$.

Entonces, por la propia definición de contractividad, se tiene que

$$
\frac{1}{M(f(x), f(y), t)}-1<k\left(\frac{1}{M(x, y, t)}-1\right)<k \eta=\delta .
$$

y, en consecuencia, por la proposición 3.3.3, $f$ es una aplicación t-uniformemente continua.

Proposición 4.2.3. Sea $(X, d)$ un espacio métrico. Entonces una aplicación $f: X \longrightarrow X$ es contractiva en el espacio métrico $(X, d)$ (con constante de contractividad $k$ ) si y sólo si $f$ es contractiva fuzzy en el espacio métrico fuzzy estándar inducido por la métrica d (con constante de contractividad $k$ ).

Demostración. Supongamos, en primer lugar, que $f$ es contractiva en el espacio métrico $(X, d)$. Entonces se tiene que $d(f(x), f(y))<k \cdot d(x, y)$. Por lo tanto 


$$
\frac{1}{M_{d}(f(x), f(y), t)}-1=\frac{d(f(x), f(y))}{t}<\frac{k d(x, y)}{t}=k\left(\frac{1}{M_{d}(x, y, t)}-1\right) .
$$

En consecuencia, $f$ es contractiva fuzzy en el espacio $\left(X, M_{d}, T_{2}\right)$.

Recíprocamente, supongamos que $f$ es contractiva fuzzy en el espacio métrico fuzzy estándar $\left(X, M_{d}, T_{2}\right)$. Entonces, para todo $x, y \in X, t>0$, se tiene que

$$
\begin{gathered}
d(f(x), f(y))=t\left(\frac{1}{M_{d}(f(x), f(y), t)}-1\right)<t k\left(\frac{1}{M_{d}(x, y, t)}-1\right)= \\
=k t\left(\frac{1}{M_{d}(x, y, t)}-1\right)=k d(x, y),
\end{gathered}
$$

y por tanto, $f$ es contractiva en $(X, d)$.

Nota 4.2.4. Recordemos que una sucesión $\left\{x_{n}\right\}_{n=1}^{\infty}$ en un espacio métrico $(X, d)$ se dice que es contractiva si existe $k \in] 0,1[$ tal que

$$
d\left(x_{n+1}, x_{n+2}\right)<k \cdot d\left(x_{n}, x_{n+1}\right), \text { para todo } n \in \mathbb{N} \text {. }
$$

Definición 4.2.5. Sea $(X, M, *)$ un espacio métrico fuzzy. Diremos que una sucesión $\left\{x_{n}\right\}_{n=1}^{\infty}$ en $X$ es contractiva fuzzy si existe $\left.k \in\right] 0,1[$ tal que

$$
\frac{1}{M\left(x_{n+1}, x_{n+2}, t\right)}-1<k\left(\frac{1}{M\left(x_{n}, x_{n+1}, t\right)}-1\right) \text {, para todo } n \in \mathbb{N}, t>0 \text {. }
$$

Proposición 4.2.6. Sea $\left(X, M_{d}, T_{2}\right)$ el espacio métrico fuzzy estándar inducido por la métrica d en $X$. Entonces una sucesión $\left\{x_{n}\right\}_{n=1}^{\infty}$ es contractiva en $(X, d)$ si y sólo si es contractiva fuzzy en $\left(X, M_{d}, T_{2}\right)$.

Demostración. Supongamos que $\left\{x_{n}\right\}_{n=1}^{\infty}$ es $d$-contractiva, es decir,

$$
d\left(x_{n+1}, x_{n+2}\right)<k \cdot d\left(x_{n}, x_{n+1}\right) \text { para todo } n \in \mathbb{N} \text {. }
$$


Entonces

$$
\begin{gathered}
\frac{1}{M_{d}\left(x_{n+1}, x_{n+2}, t\right)}-1=\frac{d\left(x_{n+1}, x_{n+2}\right)}{t}<\frac{k d\left(x_{n}, x_{n+1}\right)}{t}= \\
=k\left(\frac{1}{M_{d}\left(x_{n}, x_{n+1}, t\right)}-1\right), t>0 .
\end{gathered}
$$

Por tanto, la sucesión $\left\{x_{n}\right\}_{n=1}^{\infty}$ es contractiva fuzzy en $\left(X, M_{d}, T_{2}\right)$.

Recíprocamente, si $\left\{x_{n}\right\}_{n=1}^{\infty}$ es contractiva fuzzy, con constante de contractividad $k$, entonces, para todo $n \in \mathbb{N}, t>0$, se tiene

$$
\begin{aligned}
& d\left(x_{n+1}, x_{n+2}\right)=t\left(\frac{1}{M_{d}\left(x_{n+1}, x_{n+2}, t\right)}-1\right)< \\
& <t k\left(\frac{1}{M_{d}\left(x_{n}, x_{n+1}, t\right)}-1\right)=k d\left(x_{n}, x_{n+1}\right) .
\end{aligned}
$$

Como se quería demostrar.

En la teoría de espacios métricos es conocido que las sucesiones contractivas son sucesiones de Cauchy. De ello se deriva el siguiente resultado para los espacios métricos fuzzy estándar (aunque, como veremos más adelante, no se tiene la prueba del resultado análogo en espacios métricos fuzzy).

Corolario 4.2.7. Si $\left\{x_{n}\right\}_{n=1}^{\infty}$ es una sucesión contractiva fuzzy en un espacio métrico fuzzy estándar y completo, entonces la sucesión $\left\{x_{n}\right\}_{n=1}^{\infty}$ es convergente. 


\subsection{Teoremas de punto fijo en espacios mé- tricos fuzzy completos}

A continuación, en el siguiente resultado, vamos a extender el concocido teorema del punto fijo de Banach a aplicaciones contractivas fuzzy en espacios métricos fuzzy completos.

Teorema 4.3.1. Sea $(X, M, *)$ un espacio métrico fuzzy completo en el que toda sucesión contractiva fuzzy es una sucesión de Cauchy. Si T es una aplicación contractiva fuzzy en $X$, entonces $T$ posee un único punto fijo.

Demostración. Sean $x \in X$ y definamos por inducción la sucesión $x_{n}=$ $T^{n}(x)$, para todo $n \in \mathbb{N}$.

Supongamos que

$$
\frac{1}{M(T(x), T(y), t)}-1<k\left(\frac{1}{M(x, y, t)}-1\right),
$$

para cierto $k \in] 0,1[$, y para todo $x, y \in X, t>0$. Se tiene entonces que

$$
\frac{1}{M\left(T(x), T^{2}(x), t\right)}-1<k\left(\frac{1}{M\left(x, x_{1}, t\right)}-1\right)
$$

y, por inducción,

$$
\frac{1}{M\left(x_{n+1}, x_{n+2}, t\right)}-1<k\left(\frac{1}{M\left(x_{n}, x_{n+1}, t\right)}-1\right) \text {, para todo } n \in \mathbb{N} .
$$

En consecuencia, la sucesión $\left\{x_{n}\right\}_{n=1}^{\infty}$ es contractiva fuzzy y, por lo tanto, es de Cauchy y convergente a un punto $y \in X$.

Veamos que $y$ es un punto fijo de la aplicación $T$. Se tiene

$$
\frac{1}{M\left(T(y), T\left(x_{n}\right), t\right)}-1<k\left(\frac{1}{M\left(y, x_{n}, t\right)}-1\right)
$$


Esta última expresión tiende a cero cuando $n$ tiende a infinito. Así pues,

$$
\lim _{n \rightarrow \infty} M\left(T(y), T\left(x_{n}\right), t\right)=1, \forall t>0 .
$$

Por tanto, $\lim _{n \rightarrow \infty} T\left(x_{n}\right)=T(y)$, es decir, $\lim _{n \rightarrow \infty} x_{n+1}=T(y)$ de lo cual se deduce que

$$
T(y)=y
$$

Para probar la unicidad, supongamos que existe $z \in X$ tal que $T(z)=z$

Entonces, para $t>0$ se tiene

$$
\begin{gathered}
\frac{1}{M(y, z, t)}-1=\frac{1}{M(T(y), T(z), t)}-1<k\left(\frac{1}{M(y, z, t)}-1\right)= \\
=k\left(\frac{1}{M(T(y), T(z), t)}-1\right)<\cdots<k^{n}\left(\frac{1}{M(y, z, t)}-1\right),
\end{gathered}
$$

y la última expresión converge a cero. Por tanto, $M(x, y, t)=1$ y en consecuencia $y=z$.

Supongamos que $\left(X, M_{d}, T_{2}\right)$ es un espacio métrico fuzzy estándar completo inducido por la métrica $d$ en $X$. En virtud de la proposición 2.2.13, el espacio $(X, d)$ es completo y, por tanto, si $\left\{x_{n}\right\}_{n=1}^{\infty}$ es una sucesión contractiva fuzzy, por la proposicion 4.2.6, dicha sucesión es contractiva en $(X, d) \mathrm{y}$, por lo tanto, convergente. En consecuencia, el teorema anterior nos proporciona el siguiente corolario, que puede considerarse como la versión fuzzy del teorema clásico de contractividad de Banach en espacios métricos completos.

Corolario 4.3.2. Sea $\left(X, M_{d}, T_{2}\right)$ un espacio métrico fuzzy estándar completo, y sea $T: X \longrightarrow X$ una aplicación contractiva fuzzy. Entonces $T$ posee un único punto fijo. 
El siguiente ejemplo proporciona dos métricas fuzzy en un espacio métrico fuzzy $X$ que inducen la misma topología, pero sólo una de ellas satisface las condiciones del teorema anterior.

Ejemplo 4.3.3. Sea el espacio métrico fuzzy $\left(X, M, T_{2}\right)$, donde $X=\mathbb{N}$ y $M$ viene dada por la expresión

$$
M(x, y, t)=\left\{\begin{array}{lll}
\frac{x}{y} & \text { si } & x \leq y \\
\frac{y}{x} & \text { si } & y \leq x
\end{array}\right.
$$

siendo $x, y \in X, t>0$.

Entonces, como se ha probado anteriormente, $\left(X, M, T_{2}\right)$ es un espacio métrico fuzzy (no estándar).

Además $M$ induce en $X$ la topología discreta. De hecho, para $x \neq y$ tenemos que

$$
M(x, y, t) \leq \frac{x}{x+1}
$$

Entonces, si tomamos $r$ tal que $0<r<1-\frac{x}{x+1}$, tenemos que $y \in$ $B(x, r, t)$ si y sólo si $M(x, y, t)>1-r>\frac{x}{x+1} y$, por lo tanto, $B(x, r, t)=$ $\{x\}$.

Por otra parte, es fácil comprobar que el conjunto fuzzy $P$ de $\left.X^{2} \times\right] 0, \infty[$ dado por

$$
P(x, y, t)=\left\{\begin{array}{ccc}
\frac{1}{2} & \text { si } & x \neq y \\
1 & \text { si } & x=y
\end{array}\right.
$$

junto con la t-norma continua $T_{2}$, es una métrica fuzzy en $X$ que también induce la topología discreta en $X$. En efecto, si tomamos $0<r<\frac{1}{2}$, entonces 
$B(x, r, t)=\left\{y \in X: P(x, y, t)>\frac{1}{2}\right\}=\{x\}$.

Veamos que, si $\left\{x_{n}\right\}_{n=1}^{\infty}$ es una $M$-sucesión de Cauchy en el conjunto $X$, entonces existen $c \in C$ y $n_{0} \in \mathbb{N}$ tales que $x_{n}=c$ para todo $n \geq n_{0}$.

Supongamos que $\left\{x_{n}\right\}_{n=1}^{\infty}$ es una M-sucesión de Cauchy y que no se cumple la anterior condición. Distinguiremos dos casos:

(a) Existe $n_{0} \in \mathbb{N}$ tal que $x_{n} \in\left\{i_{1}, \ldots, i_{k}\right\}$ para todo $n \geq n_{0}$, donde

$$
i_{1}<i_{2}<\cdots<i_{k}
$$

Sea $i=\operatorname{máx}\left\{\frac{i_{s}}{i_{t}}: s, t=1,2, \ldots, k ; s<t\right\}<1$.

Si tomamos $\varepsilon>0$ tal que $1-\varepsilon>i$, y $t>0$, entonces

$$
M\left(x_{n}, x_{m}, t\right) \leq i<1-\varepsilon
$$

para cualesquiera $n, m \geq n_{0} y$, en consecuencia, $\left\{x_{n}\right\}_{n=1}^{\infty}$ no es una $M$-sucesión de Cauchy.

(b) Supongamos que, para cada $m \in \mathbb{N}$, el conjunto $A_{m}$ dado por

$$
A_{m}=\left\{x_{n}: n>m\right\} \subset \mathbb{N}
$$

es infinito.

Para $m \in \mathbb{N}$, sea $x_{m_{0}}$ el primer elemento de $A_{m}$.

Entonces, dado $\varepsilon>0$ podemos encontrar, para cada $m \in \mathbb{N}$, un elemento $x_{s} \in A_{m}(m<s)$ tal que

$$
\frac{x_{m_{0}}}{x_{s}}<1-\varepsilon
$$


es decir, $M\left(x_{m_{0}}, x_{s}, t\right)<1-\varepsilon y$, por tanto, $\left\{x_{n}\right\}_{n=1}^{\infty}$ no es una $M$ sucesión de Cauchy.

Es claro, pues, que la familia de $M$-sucesiones de Cauchy coincide con la familia de P-sucesiones de Cauchy, y es obvio que ambos espacios, $\left(X, M, T_{2}\right)$ y $\left(X, P, T_{2}\right)$, son completos.

Es fácil verificar que una sucesión $\left\{x_{n}\right\}_{n=1}^{\infty}$ es P-contractiva fuzzy si y sólo si existe $c \in X$ tal que $x_{n}=c$, para todo $n \geq 2$.

Entonces se tiene que una sucesión $P$-contractiva fuzzy es una $P$ sucesión de Cauchy $y$, en consecuencia, la métrica fuzzy P satisface las condiciones del teorema anterior.

Por otra parte, es claro que una sucesión P-contractiva fuzzy es $M$ contractiva fuzzy, pero el inverso es falso. De hecho, la sucesión 1,3, 6, 6, $6, \ldots$ es $M$-contractiva fuzzy pero no es P-contractiva fuzzy. Más aún, si la "sucesión finita" satisface la condición de ser $M$-contractiva fuzzy, entonces $x_{1}, x_{2}, \ldots, x_{n}, x_{n}, x_{n}, \ldots$ es $M$-contractiva fuzzy (pero no es $P$-contractiva fuzzy si $x_{2} \neq x_{3}$ ), aunque no sabemos si el inverso es o no cierto $y$, por tanto, no podemos asegurar, en este caso, que toda sucesión $M$-contractiva fuzzy es una $M$-sucesión de Cauchy.

El siguiente resultado es una generalización del teorema anterior y en él se da un teorema de punto fijo común para una familia numerable de aplicaciones contractivas fuzzy de un espacio métrico fuzzy. (En [52] se prueba una generalización del teorema de Grabiec en los mismos términos para espacios métricos fuzzy en el sentido de Kramosil y Michalek).

Teorema 4.3.4. Sea $(X, M, *)$ un espacio métrico fuzzy completo donde todas las sucesiones contractivas fuzzy son sucesiones de Cauchy. Sea $\left\{T_{n}\right\}_{n=1}^{\infty}$ una familia numerable de aplicaciones tal que 


$$
\frac{1}{M\left(T_{i}^{m}(x), T_{j}^{m}(y), t\right)}-1<\alpha_{i j}\left(\frac{1}{M(x, y, t)}-1\right)
$$

$0<\alpha_{i j}<k<1, t>0, m \in \mathbb{N}$. Siendo $T_{i}^{m}(x)=\overbrace{T_{i} \circ \cdots \circ T_{i}}^{m \text { veces }}(x)$.

Entonces la familia (numerable) $\left\{T_{n}\right\}_{n=1}^{\infty}$ posee un único punto fijo común.

Demostración. Sea $x_{0} \in X, m \in \mathbb{N}$. Tomamos

$$
\begin{aligned}
x_{1} & =T_{1}^{m}\left(x_{0}\right) \\
x_{2} & =T_{2}^{m}\left(x_{1}\right) \\
& \vdots \\
x_{n} & =T_{n}^{m}\left(x_{n-1}\right)
\end{aligned}
$$

Veamos que la sucesión $\left\{x_{n}\right\}_{n=1}^{\infty}$ es una sucesión contractiva fuzzy. Para $n \in \mathbb{N}$ se tiene que :

$$
\begin{gathered}
\frac{1}{M\left(x_{n+1}, x_{n+2}, t\right)}-1=\frac{1}{M\left(T_{n+1}^{m}\left(x_{n}\right), T_{n+2}^{m}\left(x_{n+1}\right), t\right)}-1< \\
<\alpha_{(n+1)(n+2)}\left(\frac{1}{M\left(x_{n}, x_{n+1}, t\right)}-1\right)
\end{gathered}
$$

Por lo tanto, $\left\{x_{n}\right\}_{n=1}^{\infty}$ es una sucesión contractiva fuzzy y, por hipótesis, es una sucesión de Cauchy, por lo que es convergente.

Sea $y=\lim _{n \rightarrow \infty}\left\{x_{n}\right\}_{n=1}^{\infty}$. Veamos que $y$ es un punto periódico de $T_{i}$, es decir, que

$$
T_{i}^{m}(y)=y, i=1,2, \ldots
$$

Se tiene que

$$
\frac{1}{M\left(T_{i}^{m}(y), T_{n+1}^{m}\left(x_{n}\right), t\right)}-1<\alpha_{i(n+1)}\left(\frac{1}{M\left(y, x_{n}, t\right)}-1\right),
$$


y la última expresión tiende a 0 cuando $n$ tiende a $\infty$.

Por lo tanto, $\lim _{n \rightarrow \infty} M\left(T_{i}^{m}(y), T_{n+1}^{m}\left(x_{n}\right), t\right)=1$ y ello implica

$$
\lim _{n \rightarrow \infty} T_{n+1}^{m}\left(x_{n}\right)=T_{i}^{m}(y) .
$$

En consecuencia $\lim _{n \rightarrow \infty} x_{n+1}=T_{i}^{m}(y)$ y, por tanto, $y=T_{i}^{m}(y)$

Veamos, a continuación, que y es el único punto periódico común.

Supongamos que existe $z \in X$ tal que $T_{i}^{m}(z)=z$. Entonces

$$
\begin{gathered}
\frac{1}{M(y, z, t)}-1=\frac{1}{M\left(T_{i}^{m}(y), T_{i}^{m}(z), t\right)}-1<\alpha_{i i}\left(\frac{1}{M(y, z, t)}-1\right)= \\
=\alpha_{i i}\left(\frac{1}{M\left(T_{i}^{m}(y), T_{i}^{m}(z), t\right)}\right)<\alpha_{i i}^{2}\left(\frac{1}{M(y, z, t)}-1\right)<\cdots \\
\cdots<\alpha_{i i}^{n}\left(\frac{1}{M(y, z, t)}-1\right)
\end{gathered}
$$

y la última expresión tiende a 0 cuando $n$ tiende a $\infty$. Por tanto $M(y, z, t)=1$ y ello implica que $y=z$

Por último, $T_{i}(y)=T_{i}\left(T_{i}^{m}(y)\right)=T_{i}^{m}\left(T_{i}(y)\right)$. En consecuencia, $T_{i}(y)$ es un punto periódico y, por la unicidad probada anteriormente, se obtiene que $y$ es un punto fijo común, es decir $T_{i}(y)=y$.

El siguiente teorema utiliza el concepto de aplicación contractiva dado por Grabiec [23] con la intención de extender el teorema del punto fijo de Banach a este tipo de aplicaciones contractivas en espacios métricos fuzzy completos. Cabe destacar que, tanto la prueba como la condición inicial impuesta en este teorema, son muy distintas a las dadas por Grabiec debido a que la definición de sucesión de Cauchy dada por Grabiec (definicion 4.4.1) es más débil que la definición 1.2.6 y, por lo tanto, el argumento desarrollado en el trabajo de 
Grabiec no asegura que la sucesión construida sea de Cauchy en el sentido más fuerte de George y Veeramani.

Nota 4.3.5. El producto infinito $M\left(x, y, t_{1}\right) * \cdots * M\left(x, y, t_{n}\right) * \cdots$ en el espacio métrico fuzzy $(X, M, *)$ lo denotaremos $\prod_{i=1}^{\infty} M\left(x, y, t_{i}\right)$.

Recordemos que el producto infinito usual de números reales $\prod_{i=1}^{\infty} u_{n}$, se dice que es convergente si la sucesión de los productos parciales $\pi_{n}=\prod_{m=1}^{n} u_{m}$ converge a un número real distinto de cero. En este caso, la sucesión de los restos $R_{m}=\prod_{n=m+1}^{\infty} u_{n}$ converge a 1 .

Definición 4.3.6. Diremos que una sucesión de números reales positivos $\left\{t_{n}\right\}_{n=1}^{\infty}$ es s-creciente si existe $n_{0} \in \mathbb{N}$ tal que $t_{n}+1 \leq t_{n+1}$, para todo $n \geq n_{0}$.

Teorema 4.3.7. (Teorema de contractividad de Banach fuzzy) Sea $(X, M, *)$ un espacio métrico fuzzy completo de manera que dado $\varepsilon>0$, para cada $x, y \in X$ y para cada sucesión $\left\{t_{n}\right\}_{n=1}^{\infty}$ s-creciente, existe $n_{0} \in \mathbb{N}$ tal que

$$
\prod_{n=n_{0}}^{\infty} M\left(x, y, t_{n}\right)>1-\varepsilon
$$

$\left(O\right.$, lo que es lo mismo, $\prod_{n=1}^{\infty} M\left(x, y, t_{n}\right)$ converge cuando $*$ es el producto usual en el intervalo $[0,1])$.

Sea $k \in] 0,1[$ y sea $T: X \longrightarrow X$ una aplicación que cumple la siguiente condición, para cualesquiera $x, y \in X, t>0$ :

$$
M(T(x), T(y), k t) \geq M(x, y, t)
$$

Entonces $T$ posee un único punto fijo. 
Demostración. Fijemos $x \in X$. Definamos por inducción $x_{n}=T^{n}(x), n=$ $1,2, \ldots$

Pata $t>0$, tenemos que

$$
M\left(x_{1}, x_{2}, t\right)=M\left(T(x), T^{2}(x), t\right) \geq M\left(x, T(x), \frac{t}{k}\right)=M\left(x, x_{1}, \frac{t}{k}\right)
$$

Por inducción, es fácil demostrar que se tiene

$$
M\left(x_{n}, x_{n+1}, t\right) \geq M\left(x, x_{1}, \frac{t}{k^{n}}\right), n=1,2, \ldots
$$

Sean $t>0, \varepsilon>0$. Sean $m, n \in \mathbb{N}$, y supongamos (sin pérdida de generalidad) que $n<m$. Si tomamos $s_{i}>0, i=n, n+1, \ldots, m-1$ que satisfacen $s_{n}+\cdots+s_{m-1} \leq 1$, entonces,

$$
\begin{gathered}
M\left(x_{n}, x_{m}, t\right) \geq M\left(x_{n}, x_{n+1}, s_{n} t\right) * \cdots * M\left(x_{m-1}, x_{m}, s_{m-1} t\right) \geq \\
\geq M\left(x, x_{1}, \frac{s_{n} t}{k^{n}}\right) * \cdots * M\left(x, x_{1}, \frac{s_{m-1} t}{k^{m-1}}\right)
\end{gathered}
$$

En particular, como $\sum_{n=1}^{\infty} \frac{1}{n(n+1)}=1$, podemos tomar

$$
s_{i}=\frac{1}{i(i+1)}, i=n, n+1, \ldots, m-1,
$$

y entonces,

$$
\begin{gathered}
M\left(x_{n}, x_{m}, t\right) \geq M\left(x, x_{1}, \frac{t}{n(n+1) k^{n}}\right) * \cdots * M\left(x, x_{1}, \frac{t}{(m-1) m k^{m-1}}\right) \geq \\
\geq \prod_{n=1}^{\infty} M\left(x, x_{1}, \frac{t}{n(n+1) k^{n}}\right) .
\end{gathered}
$$

Ahora bien, la sucesión de término general $t_{n}=\frac{t}{n(n+1) k^{n}}$ es $s$-creciente. 
Para probar nuestra afirmación consideraremos la sucesión $\left\{t_{n+1}-t_{n}\right\}_{n=1}^{\infty} \mathrm{y}$ veremos que ésta diverge a infinito. En efecto,

$$
\begin{gathered}
t_{n+1}-t_{n}=\frac{t}{(n+1)(n+2) k^{n+1}}-\frac{t}{n(n+1) k^{n}}= \\
=\frac{t n(n+1) k^{n}-t(n+1)(n+2) k^{n+1}}{n(n+1)^{2}(n+2) k^{2 n+1}}= \\
=\frac{t k^{n}[n-k(n+2)]}{n(n+1)(n+2) k^{n+1}}=\frac{t[(1-k) n-2 k]}{n(n+1)(n+2) k^{n+1}} .
\end{gathered}
$$

Es claro que el numerador de la anterior fracción tiende a infinito cuando $n$ tiende a infinito.

Por otra parte, el denominador de la anterior fracción converge a cero. Para demostrar esta afirmación consideraremos una serie cuyo término general es el denominador de la última fracción y probaremos que es convergente. Para verlo, utilizaremos el criterio de d'Alembert:

$$
\frac{(n+1)(n+2)(n+3) k^{n+2}}{n(n+1)(n+2) k^{n+1}}=\frac{k(n+3)}{n},
$$

y esta sucesión tiende a $k$, cuando $n$ tiende a infinito. Como $k<1$, esta serie es convergente.

Así pues, la sucesión $\left\{t_{n}\right\}_{n=1}^{\infty}$ es $s$-creciente. Por todo ello, atendiendo a la hipótesis del teorema, existe $n_{0} \in \mathbb{N}$ tal que

$$
\prod_{n=n_{0}}^{\infty} M\left(x, x_{1}, \frac{t}{n(n+1) k^{n}}\right)>1-\varepsilon
$$

y, en consecuencia, $M\left(x_{n}, x_{m}, t\right)>1-\varepsilon$ para cualesquiera $n, m \geq n_{0}$.

Así queda probado que $\left\{x_{n}\right\}_{n=1}^{\infty}$ es una sucesión de Cauchy. 
En el caso de que $*$ sea el producto usual en $(0,1]$, entonces el producto

$$
\prod_{n=1}^{\infty} M\left(x, x_{1}, \frac{t}{n(n+1) k^{n}}\right)
$$

es convergente, y, por tanto, la sucesión de restos

$$
R_{m}=\prod_{n=m+1}^{\infty} M\left(x, x_{1}, \frac{t}{n(n+1) k^{n}}\right)
$$

converge a 1 cuando $m$ tiende a $\infty$. Por tanto, existe $n_{0} \in \mathbb{N}$ tal que

$$
\prod_{n=n_{0}}^{\infty} M\left(x, x_{1}, \frac{t}{n(n+1) k^{n}}\right) \geq 1-\varepsilon
$$

y estamos otra vez en las mismas condiciones, por lo que $\left\{x_{n}\right\}_{n=1}^{\infty}$ es de Cauchy.

Como el espacio $X$ es completo, existe $y \in X$ tal que $\lim _{n \rightarrow \infty} x_{n}=y$.

Veamos que $y$ es un punto fijo de la aplicación $T$. Tenemos que, para $t>0$ :

$$
\begin{aligned}
M(T(y), y, t) & \geq M\left(T(y), T\left(x_{n}\right), \frac{t}{s}\right) * \cdots * M\left(x_{n+1}, y, \frac{t}{2}\right) \geq \\
& \geq M\left(y, x_{n}, \frac{t}{2 k}\right) * M\left(x_{n+1}, y, \frac{t}{2}\right)
\end{aligned}
$$

La anterior expresión tiende a 1 cuando $n$ tiende a infinito. Por tanto, $M(T(y), y, t)=1$ y tenemos que $T(y)=y$.

Para probar la unicidad, supongamos que $T(z)=z$ para algún $z \in X$.

Entonces

$$
1 \geq M(z, y, t)=M(T(z), T(y), t) \geq M\left(z, y, \frac{t}{k}\right)=M\left(T(z), T(y), \frac{t}{k}\right) \geq
$$




$$
\geq M\left(z, y, \frac{t}{k^{2}}\right) \geq \cdots \geq M\left(z, y, \frac{t}{k^{n}}\right)
$$

Veamos que la sucesión $t_{n}=\left\{\frac{t}{k^{n}}\right\}_{n=1}^{\infty}$ es s-creciente. En efecto,

$$
t_{n+1}-t_{n}=\frac{t}{k^{n+1}}-\frac{t}{k^{n}}=\frac{t(1-k)}{k^{n+1}}
$$

y es claro que el numerador es constante y el denominador tiende a cero, con lo que la sucesión de la diferencia entre dos términos consecutivos tiende a infinito.

Entonces, por hipótesis, dado $\varepsilon \in] 0,1\left[\right.$, existe $n_{0} \in \mathbb{N}$ tal que

$$
\prod_{n \geq n_{0}} M\left(z, y, \frac{t}{k^{n}}\right) \geq 1-\varepsilon
$$

y, claramente,

$$
\lim _{n \rightarrow \infty} M\left(z, y, \frac{t}{k^{n}}\right)=1
$$

Por lo tanto, $M(z, y, t)=1$ y, en consecuencia, $z=y$.

Nota 4.3.8. En el anterior teorema, la aplicación $T: X \longrightarrow X$ satisface, para algún $k \in] 0,1$ [, la condición (de contractividad)

$$
M(T(x), T(y), k t) \geq M(x, y, t) \text { para cualesquiera } x, y \in X, t>0
$$

Es interesante hacer notar que esta condición fue introducida por Sehgal y Bharucha-Reid [47] en el estudio de los espacios probabilísticos de Menger. La siguiente proposición prueba que tal concepto está íntimamente relacionado con el concepto de contractividad fuzzy, y es acorde, además, con la teoría clásica. 
Proposición 4.3.9. Sea $\left(X, M_{d}, T_{2}\right)$ el espacio métrico fuzzy estándar asociado al espacio métrico $(X, d)$. Sea $f: X \rightarrow X$ una aplicación y sea $k \in] 0,1[$. Entonces son equivalentes:

(i) $f$ es una aplicación contractiva en $(X, d)$ (con constante de contractivi$d a d k)$.

(ii) $f$ es una aplicación contractiva fuzzy en $\left(X, M_{d}, T_{2}\right)$ (con constante de contractividad $k$ ).

(iii) $M_{d}(f(x), f(y), k t)>M_{d}(x, y, t)$, para cualesquiera $x, y \in X, t>0$

Demostración. La equivalencia entre $(i)$ y (ii) se ha visto en la proposición 4.2.3.

Supongamos que, para $x, y \in X, t>0$, se satisface la condición

$$
M_{d}(f(x), f(y), k t)>M_{d}(x, y, t)
$$

Ello es cierto si y sólo si

$$
\frac{k t}{k t+d(f(x), f(y))}>\frac{t}{t+d(x, y)}
$$

que es equivalente a la condición

$$
\frac{k t+d(f(x), f(y))}{k t}<\frac{t+d(x, y)}{t}
$$

o lo que es lo mismo

$$
\frac{d(f(x), f(y))}{k t}<\frac{d(x, y)}{t}
$$

que ocurre si y sólo si

$$
\frac{d(f(x), f(y))}{t}<\frac{k d(x, y)}{t}
$$


o, equivalentemente,

$$
\left(\frac{t+d(f(x), f(y))}{t}-1\right)<k\left(\frac{t+d(x, y)}{t}-1\right)
$$

es decir, si y sólo si

$$
\left(\frac{1}{M_{d}(f(x), f(y), t)}-1\right)<k\left(\frac{1}{M_{d}(x, y, t)}-1\right)
$$

que es la definición de aplicación contractiva fuzzy (con constante de contractividad $k$ ).

El siguiente ejemplo trata de ilustrar el anterior teorema justificando las condiciones iniciales impuestas en él a través de dos espacios métricos fuzzy. En el primero de ellos, la métrica fuzzy asociada no cumple dichas condiciones mientras que, en cambio, en el segundo, la métrica fuzzy asociada sí las cumple.

\section{Ejemplo 4.3.10.}

(a) Consideremos el espacio métrico fuzzy completo $(X, P, *)$ del ejemplo 4.3.3. Para $x, y \in X$ tales que $x \neq y$, consideremos la sucesión estrictamente creciente de reales $\left(t_{n}\right)$ dada por $t_{n}=n$, para todo $n \in \mathbb{N}$, que, obviamente, es s-creciente. Entonces,

$$
\prod_{n=1}^{\infty} P\left(x, y, t_{n}\right)=\prod_{n=1}^{\infty} u_{n}
$$

donde $u_{n}=\frac{1}{2}$ para todo $n \in \mathbb{N}$, y, por lo tanto, la sucesión $\pi_{n}$ converge a 0 . 
En consecuencia, la métrica fuzzy $P$ no satisface la condición del teorema anterior.

Notemos que, si tomamos $T$ como la aplicación identidad (i.e., $T(x)=$ $x$, para $x \in X)$, entonces $T$ satisface la condición de contractividad

$$
P(T(x), T(y), k t) \geq P(x, y, t) \text { para cualesquiera } x, y \in X, k \in] 0,1[
$$

pero no posee un único punto fijo. (De hecho, todos los puntos de $X$ son fijos para la aplicación T).

(b) Sea $X=\mathbb{N}$ y tomamos $a * b=a b$ para cualesquiera $a, b \in[0,1]$.

Definimos el conjunto fuzzy $Q$ definido en $\left.X^{2} \times\right] 0, \infty[$ como sigue:

$$
Q(x, y, t)=\left\{\begin{array}{cc}
\frac{t^{2}}{t^{2}+1} & \text { si } x \neq y \\
1 & \text { si } x=y
\end{array}\right.
$$

para $x, y \in X, t>0$.

Es fácil demostrar que $Q$ es una métrica fuzzy en el conjunto $X$. En efecto, las propiedades (GV1), (GV2), (GV3) y (GV5) de la definición 1.3.1 son inmediatas. Además, se cumple que

$$
Q(x, y, t) * Q(y, z, s)=\frac{t^{2}}{t^{2}+1} \frac{s^{2}}{s^{2}+1} \leq Q(x, z, t+s)
$$

$y$, por tanto $\left(X, Q, T_{2}\right)$ es un espacio métrico fuzzy.

Por otra parte, se tiene que

$$
B\left(x, \frac{1}{t^{2}+1}, t\right)=\left\{y \in X: \frac{t^{2}}{t^{2}+1}>1-\frac{1}{t^{2}+1}\right\} \cup\{x\}=\{x\}
$$

y, en consecuencia, la métrica fuzzy $Q$ induce la topología discreta. 
Además, una sucesión $\left\{x_{n}\right\}_{n=1}^{\infty}$ de $X$ es una $Q$-sucesión de Cauchy si y sólo si existen $n_{0} \in \mathbb{N}$ y $c \in X$ tales que $x_{n}=c$ para todo $n \geq n_{0}$. Si $\left\{x_{n}\right\}_{n=1}^{\infty}$ es una $Q$-sucesión de Cauchy, entonces, para cualesquiera $\varepsilon \in] 0,1\left[, t>0\right.$, existe $n_{0} \in \mathbb{N}$ tal que $Q\left(x_{n}, x_{m}, t\right)>1-\varepsilon$ para $n, m \geq n_{0}$, lo cual sólo es posible si $x_{n}=x_{m}$.

En consecuencia, $(X, Q, *)$ es un espacio métrico fuzzy completo.

Sea ahora $\left\{t_{n}\right\}_{n=1}^{\infty}$ una sucesión de reales positivos s-creciente. Es obvio que

$$
\prod_{n=1}^{\infty} Q\left(x, y, t_{n}\right)=\prod_{n=1}^{\infty} \frac{t_{n}^{2}}{t_{n}^{2}+1} .
$$

Veamos que $\prod_{n=1}^{\infty} Q\left(x, y, t_{n}\right)$ es convergente para $x, y \in X, x \neq y$.

Para ello probaremos que $\prod_{n=1}^{\infty} u_{n}$ es convergente, donde

$$
\begin{array}{ll}
u_{i}=1, & i=1,2, \ldots n_{0}-1 \\
u_{n}=\frac{t_{n}^{2}+1}{t_{n}^{2}}=1+\frac{1}{t_{n}^{2}}, & n \geq n_{0}
\end{array}
$$

Tenemos que

$$
\prod_{n=1}^{\infty} u_{n}=\prod_{n=1}^{\infty}\left(1+v_{n}\right)
$$

donde $v_{n}=0$ para $n=1,2, \ldots, n_{0}-1, y v_{n}=\frac{1}{t_{n}^{2}}$ para todo $n \geq n_{0}$.

Sea $E\left(t_{n}\right)=m_{n} \in \mathbb{N}$. Entonces $t_{n} \geq m_{n} y$, por tanto,

$$
\sum_{n=1}^{\infty} v_{n} \leq \sum_{n=1}^{\infty} \frac{1}{\left(E\left(t_{n}\right)\right)^{2}}=\sum_{n=1}^{\infty} \frac{1}{m_{n}^{2}} \leq \sum_{n=1}^{\infty} \frac{1}{n^{2}}
$$

por hipótesis de $\left\{t_{n}\right\}_{n=1}^{\infty}$. 
Un criterio de comparación prueba que la serie $\sum_{n=1}^{\infty} v_{n}$ es convergente $y$, por lo tanto, $\prod_{n=1}^{\infty} u_{n}$ converge a un número real positivo $A$.

Todo ello demuestra que $\prod_{n \geq n_{0}} \frac{t_{n}^{2}}{t_{n}^{2}+1}$ converge a $\frac{1}{A} y$, en consecuencia, $\prod_{n=1}^{\infty} Q\left(x, y, t_{n}\right)$ converge.

Hemos probado, pues, que la métrica fuzzy $Q$ satisface las condiciones del teorema anterior.

Nota 4.3.11. Cabe hacer notar que, en el segundo caso del ejemplo anterior, la aplicación identidad no satisface la condición de contractividad

$$
Q(T(x), T(y), k t) \geq Q(x, y, t) \text { para cualesquiera } x, y \in X, k \in] 0,1[\text {. }
$$

\subsection{Teoremas de punto fijo en espacios mé- tricos fuzzy de Kramosil y Michalek com- pletos en el sentido de Grabiec}

A lo largo de todo este apartado, la terna $(X, M, *)$ será un espacio métrico fuzzy según la definición aportada por Kramosil y Michalek (definición 1.2.10).

Nuestro objetivo es extender el teorema de punto fijo de Banach a aplicaciones contractivas fuzzy en espacios métricos fuzzy completos en el sentido aportado por Grabiec [23]. Las siguientes definiciones y resultados se encuentran en [19]. 
Definición 4.4.1. La sucesión $\left\{x_{n}\right\}_{n=1}^{\infty}$ en un espacio métrico fuzzy $(X, M, *)$ es de Cauchy en el sentido de Grabiec (y lo denotaremos G-Cauchy) si

$$
\lim _{n \rightarrow \infty} M\left(x_{n+p}, x_{n}, t\right)=1 \text {, para cualesquiera } t>0, p \in \mathbb{N} .
$$

Proposición 4.4.2. Una sucesión $\left\{x_{n}\right\}_{n=1}^{\infty}$ en $X$ es convergente a $x \in X$ si se cumple

$$
\lim _{n \rightarrow \infty} M\left(x_{n}, x, t\right)=1 \text { para todo } t>0 .
$$

Un espacio métrico fuzzy donde toda sucesión $G$-Cauchy es convergente diremos que es completo en el sentido de Grabiec (y lo denotaremos $G$ completo).

Además, el espacio métrico fuzzy $(X, M, *)$ se dice que es compacto si toda sucesión contiene una subsucesión convergente.

Nota 4.4.3. Las definiciones 4.2.1 y 4.2.5 de aplicación contractiva fuzzy y de sucesión contractiva fuzzy, respectivamente, continúan siendo válidas, aunque no seamos capaces de dotar un espacio métrico fuzzy de Kramosil y Michalek de una estructura uniforme.

El siguiente teorema demuestra la existencia de un único punto fijo en aplicaciones contractivas fuzzy de espacios métricos fuzzy (en el sentido de Kramosil y Michalek) con la condición de Cauchy aportada por Grabiec.

Teorema 4.4.4. (Teorema de contractividad fuzzy de Banach). Sea $(X, M, *)$ un espacio métrico fuzzy $G$-completo y sea $T: X \longrightarrow X$ una aplicación contractiva fuzzy. Entonces $T$ posee un único punto fijo.

Demostración. Supongamos que $T$ satisface la condición de contractividad

$$
\frac{1}{M(T(x), T(y), t)}-1<k\left(\frac{1}{M(x, y, t)}-1\right)
$$


para cierto $k \in] 0,1[\mathrm{y}$ para todo $x, y \in X, t>0$.

Sea $x \in X$. Definimos por inducción $x_{n}=T^{n}(x)$, para todo $n \in \mathbb{N}$.

Ya se ha visto con anterioridad, en el teorema 4.3.1, que $\left\{x_{n}\right\}_{n=1}^{\infty}$ es una sucesión contractiva fuzzy que satisface

$$
\frac{1}{M\left(x_{n+1}, x_{n+2}, t\right)}-1<k\left(\frac{1}{M\left(x_{n}, x_{n+1}, t\right)}-1\right) \text {, para todo } n \in \mathbb{N}
$$

Entonces,

$$
\begin{aligned}
& \frac{1}{M\left(x_{n+1}, x_{n+2}, t\right)}-1<k^{2}\left(\frac{1}{M\left(x_{n-1}, x_{n}, t\right)}-1\right)<\ldots< \\
&<k^{n}\left(\frac{1}{M\left(x_{1}, x_{2}, t\right)}-1\right)
\end{aligned}
$$

Esta última expresión tiende a cero cuando $\mathrm{n}$ tiende a infinito $\mathrm{y}$, por lo tanto,

$$
\lim _{n \rightarrow \infty} M\left(x_{n}, x_{n+1}, t\right)=1 \text {, para todo } t>0
$$

Si fijamos $p \in \mathbb{N}$, se tiene que

$$
M\left(x_{n}, x_{n+p}, t\right) \geq M\left(x_{n}, x_{n+1}, \frac{t}{p}\right) * \cdots * M\left(x_{n+p-1}, x_{n+p}, \frac{t}{p}\right)
$$

que tiende a $1 * \cdots * \cdots * 1=1$, y, en consecuencia, $\left\{x_{n}\right\}_{n=1}^{\infty}$ es una sucesión $G$ Cauchy. Por tanto, $\left\{x_{n}\right\}_{n=1}^{\infty}$ converge a algún $y \in X$.

De manera análoga a la demostración del Teorema 4.3.1 se prueba que $y$ es el único punto fijo de la aplicación $T$.

En el Teorema 4.4.4 se ha probado que toda sucesión contractiva fuzzy es $G$-Cauchy. Ahora bien, el siguiente resultado permanece pendiente de demostración. 
Cuestión abierta 4.1. ¿Es una sucesión contractiva fuzzy una sucesión de Cauchy en el sentido de George y Veeramani?

Nota 4.4.5. En [23] se demuestra el siguiente teorema de contractividad de Edelstein:

"Sea $(X, M, *)$ un espacio métrico fuzzy compacto y sea $T: X \longrightarrow X$ una aplicación que satisface que $M(T(x), T(y), \cdot)>M(x, y, \cdot)$ para cualesquiera $x, y \in X$ tales que $x \neq y$. Entonces $T$ posee un único punto fijo."

Si consideramos que la aplicación $T: X \longrightarrow X$ es contractiva fuzzy satisfaciendo que

$$
\frac{1}{M(T(x), T(y), t)}-1<k\left(\frac{1}{M(x, y, t)}-1\right)
$$

para cierto $k \in] 0,1[y$ para todo $x, y \in X, t>0$. Entonces

$$
M(T(x), T(y), t)>M(x, y, t) \text { si } x \neq y
$$

y, por lo tanto, el mencionado teorema de contractividad de Edelstein se satisface para aplicaciones contractivas fuzzy. 


\section{Bibliografía}

[1] G. Artico, R. Moresco, On fuzzy metrizability, J. Math. Anal. Appl. 107 (1985), 144-147.

[2] K. Atanassov, Intuitionistic fuzzy sets, V.Sgurev, ed., VII ITKR's Session, Sofia (June 1983 Central Sci. and Techn. Library, Bulg. Academy of Sciences), 1984.

[3] K. Atanassov, Intuitionistic fuzzy sets, Fuzzy Sets and Systems 20 (1968), 87-96.

[4] K. Atanassov, Review and new results on intuitionistic fuzzy sets, Preprint IM-MFAIS-1-88, Sofia (1988).

[5] G. BeER, Topologies on Closed and Closed Convex Sets, Kluwer Acad. Publ., Dordrecht, 1993.

[6] G. L. CAIN JR., R. H. KASRIEL, Fixed and periodic points of local contraction mappings on probabilistic metric spaces, Mathematical Systems Theory 9 (1976), 289-297.

[7] C. L. Chang, Fuzzy topological spaces, J. Math. Anal. Appl. 24 (1968), 182-190. 
[8] K. C. Chattopadhyay, R. N. Hazra, S. K. Samanta, Gradation of openess: fuzzy topology, Fuzzy Sets and Systems 49 (1992), 237-242.

[9] D. ÇOKER, An introduction to intuitionistic fuzzy topological spaces, Fuzzy Sets and Systems 88 (1997), 81-89.

[10] Deng ZI-Ke, Fuzzy pseudo metric spaces, J. Math. Anal. Appl. 86 (1982), 74-95.

[11] Deng ZI-Ke, Separation axioms for completeness and total boundedness in fuzzy pseudometric spaces, J. Math. Anal. Appl. 112 (1985), 141-150.

[12] M. Edelstein, On fixed and periodic points under contractive mappings, J. London Math Soc. 37 (1962), 74-79.

[13] P. Eklund, W. GÄHLER, Basic notions for fuzzy topology I, II, Fuzzy Sets and Systems 26 (1988), 333-356; 27 (1988) 171-195.

[14] R. Engelking, General Topology, PWN-Polish Sci. Publ., Warsawa, 1977.

[15] M. A. ERceg, Metric spaces in fuzzy set theory, J. Math. Anal. Appl. 69 (1979), 205-230.

[16] J. X. FAng, On fixed point theorems in fuzzy metric spaces, Fuzzy Sets and Systems 46 (1992), 107-113.

[17] P. Fletcher, W. F. Lindgren, Quasi-Uniform Spaces, Marcel Dekker, New York, 1982.

[18] J. Goguen, L-fuzzy sets, J. Math. Anal. Appl. 18 (1967), 145-174.

[19] A. George, P. Veeramani, On some results in fuzzy metric spaces, Fuzzy Sets and Systems 64 (1994), 395-399. 
[20] A. George, P. Veeramani, On some results of analysis for fuzzy metric spaces, Fuzzy Sets and Systems 90 (1997), 365-368.

[21] A. George, P. Veeramani, Some theorems in fuzzy metric spaces, J. Fuzzy Math. 3 (1995), 933-940.

[22] A. George, P. Veeramani, Compact and bounded sets in fuzzy metric spaces, J. Fuzzy Math. 8 (2000), 975-980.

[23] M. Grabiec, Fixed points in fuzzy metric spaces, Fuzzy Sets and Systems 27 (1988), 385-389.

[24] V. Gregori, S. Romaguera, Some properties of fuzzy metric spaces, Fuzzy Sets and Systems 115 (2000), 485-489.

[25] V. Gregori, S. Romaguera, On completion of fuzzy metric spaces, submitted.

[26] V. Gregori, S. Romaguera, A. Sapena, Uniform continuity in fuzzy metric spaces. Rend. Istit. Mat. Univ. Trieste, to appear.

[27] V. Gregori, A. Sapena, On fixed point theorems in fuzzy metric spaces, Fuzzy Sets and Systems, 125 (2002), 245-252.

[28] O. HADZIC, Fixed point theorems for multivalued mappings in some classes of fuzzy metric spaces, Fuzzy Sets and Systems 29 (1989), 115125.

[29] R. N. Hazra, S. K. Samanta, K. C. Chattopadhyay, Fuzzy topology redefined, Fuzzy Sets and Systems 45 (1992), 79-82.

[30] H. Hu, Fuzzy topological spaces, J. Math. Anal. Appl. 110 (1985), 141178. 
[31] B. Hutton, Products of fuzzy topological spaces, Topology Appl. 11 (1980), 59-67.

[32] I. Jermolaeva, On a Hausdorffness function of a fuzzy topological space, Zbornik Radova Filozof. Fakulteta u Nižu. Ser. Mat. 2 (1988), 73.

[33] O. Kaleva, S. Seikkala, On fuzzy metric spaces, Fuzzy Sets and Systems 12 (1984), 215-229.

[34] J.C. Kelley, General Topology, Van Nostrand, 1955.

[35] J. Kramosil, J. Michalek, Fuzzy metric and statistical metric spaces, Kybernetika 11 (1975), 326-334.

[36] R. Lowen, Fuzzy topological spaces and fuzzy compactness, J. Math. Anal. Appl. 56 (1976), 621-633.

[37] R. Lowen, Initial and final fuzzy topologies and the fuzzy Tychonoff theorem, J. Math. Anal. Appl. 58 (1977), 11-21.

[38] K. Menger, Statistical metrics, Proc. Nat. Acad. Sci., U.S.A. 28 (1942), 535-537.

[39] S. N. Mishra, N. Sharma, S. L. Singh, Common fixed points of maps on fuzzy metric spaces, Int. J. Math. and Math. Sci. 17 (1994), 253-258.

[40] E. PAP, O. HADZIC, R. Mesiar, A fixed point theorem in probabilistic metric spaces and an application, J. Math. Anal. Appl. 202 (1996), 433449.

[41] E. Parau, V. RAdu, Some remarks on Tardiff's fixed point theorem on Menger spaces, Portugal. Math. 54 (1997), 431-440. 
[42] V. RAFU, Some fixed point theorems in probabilistic metric spaces, stability problems for stochastic models, Lecture Notes in Mathematics, vol. 1233, Springer, Berlin, (1987) 125-133.

[43] A. Sapena, A contribution to the study of fuzzy metric spaces, Appl. Gen. Topology 2 (2001), 63-76.

[44] B. Schweizer, H. Sherwood, R. M. TArdiff, Contractions on probabilistic metric spaces: examples and counterexamples, Stochastica XII (1988), 5-17.

[45] B. Schweizer, A. Sklar, Statistical metric spaces, Pacific. J. Math. 10 (1960), 314-334.

[46] B. Schweizer, A. Sklar, Probabilistic Metric Spaces, Elsevier Science Publishing Co., New York, 1983.

[47] V. M. Sehgal, A. T. Bharucha-Reid, Fixed points of contraction mappings of probabilistic metric spaces, Math. Systems Theory 6 (1972), 97-102.

[48] A. P. Šostak, On a fuzzy topological structure, Suppl. Rend. Circ. Mat. Palermo Ser II, 11 (1985) 89-103.

[49] A. P. Šostak, Two decades of fuzzy topology: basic ideas, notions and results, Uspekhi Mat. Nauk 44 (1989), 99-147.

[50] P. V. Subrahmanyam, A common fixed point theorem in fuzzy metric spaces, Information Sciences 83 (1995), 103-112.

[51] R. M. TARDIFF, Contraction maps on probabilistic metric spaces, J. Math. Anal. Appl. 165 (1992), 517-523. 
[52] R. VAsukI, A common fixed point theorem in a fuzzy metric space, Fuzzy Sets and Systems 97 (1998), 395-397.

[53] A. Wilansky, Topology for Analysis, Ed. Ginn, New York, 1970.

[54] L.A. ZADEH, Fuzzy sets, Inform. Control 8 (1965), 338-353. 